\title{
Photoresponsive Polymersomes as Smart, Triggerable Nanocarriers
}

\author{
Inauguraldissertation \\ zur \\ Erlangung der Würde eines Doktors der Philosophie \\ vorgelegt der \\ Philosophisch-Naturwissenschaftlichen Fakultät \\ der Universität Basel \\ von \\ Etienne Cabane \\ aus \\ Frankreich

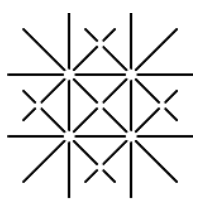 \\ U N I \\ B A S E L
}

Basel, 2013

Original document stored on the publication server of the University of Basel edoc.unibas.ch

This work is licenced under the agreement „Attribution Non-Commercial No Derivatives - 2.5 Switzerland“. The complete text may be viewed here: creativecommons.org/licenses/by-nc-nd/2.5/ch/deed.en 


\section{creative}

\section{Attribution-Noncommercial-No Derivative Works 2.5 Switzerland}

\section{You are free:}

to Share - to copy, distribute and transmit the work

\section{Under the following conditions:}

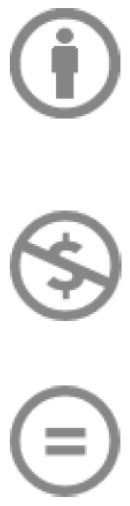

Attribution. You must attribute the work in the manner specified by the author or licensor (but not in any way that suggests that they endorse you or your use of the work).

Noncommercial. You may not use this work for commercial purposes.

No Derivative Works. You may not alter, transform, or build upon this work.

- For any reuse or distribution, you must make clear to others the license terms of this work. The best way to do this is with a link to this web page.

- Any of the above conditions can be waived if you get permission from the copyright holder.

- Nothing in this license impairs or restricts the author's moral rights.

\section{Your fair dealing and other rights are in no way affected by the above.}

This is a human-readable summary of the Legal Code (the full license) available in German: http://creativecommons.org/licenses/by-nc-nd/2.5/ch/legalcode.de

\section{Disclaimer:}

The Commons Deed is not a license. It is simply a handy reference for understanding the Legal Code (the full license) - it is a human-readable expression of some of its key terms. Think of it as the user-friendly interface to the Legal Code beneath. This Deed itself has no legal value, and its contents do not appear in the actual license. Creative Commons is not a law firm and does not provide legal services. Distributing of, displaying of, or linking to this Commons Deed does not create an attorney-client relationship. 
Genehmigt von der Philosophisch-Naturwissenschaftlichen Fakultät auf Antrag von

Prof. Dr. Wolfgang Meier

und

PD Dr. Cornelia G. Palivan

Basel, den 21.06.2011

Prof. Dr. Martin Spiess

Dekan 
iv 


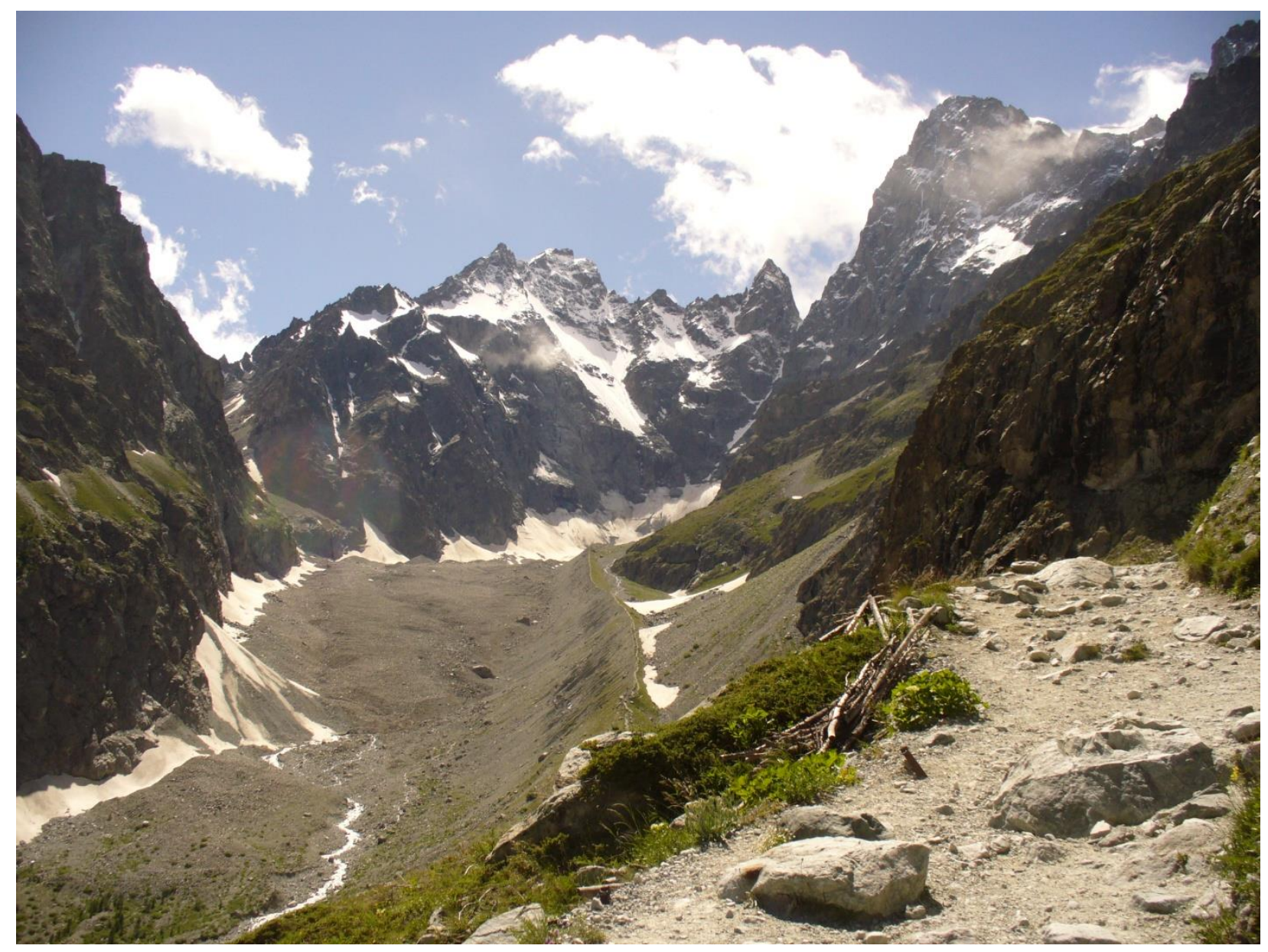

"If you don't know where you are going, any road will get you there." Lewis Caroll 
vi | 


\section{Abstract}

Nowadays, conventional drug delivery systems (DDS), such as oral and parenteral delivery, are the most used administration routes for simple drug molecules. Oral delivery is the most desirable route, especially regarding patient compliance since it is a non-invasive technique, but also regarding cost effectiveness, and ease of use. However, it is clear that the technique is subjected to many hurdles: fragile molecules, such as therapeutic proteins, may lose part of their activity before they can reach the targeted site in the body, because they can be degraded by enzymes or harsh $\mathrm{pH}$ conditions, or simply screened out by immunogenic recognition in blood circulation. Additionally, the technique suffers from a lack of control over the release rate, which may be an important drawback, especially regarding toxicity issues.

Therefore, with the increasing complexity of therapeutic agents available to treat a variety of conditions, the need for sophisticated drug delivery systems to improve their liberation to the body is growing. Among the requirements for intelligent DDS, responsiveness is highly desirable as a mean to control pharmacokinetics and pharmacodynamics.

In this thesis, we study the potential of polymeric vesicles obtained from the selfassembly of a photocleavable amphiphilic block copolymer as a light-triggered DDS.

The amphiphilic poly(methyl caprolactone)-ONB-poly(acrylic acid) copolymer (PMCL$O N B$-PAA), bearing a photosensitive O-nitrobenzyl linker located as the junction point between the two blocks, is synthesized by the combination of two living polymerization techniques, ring opening polymerization (ROP) and atom transfer radical polymerization (ATRP). The photocleavable linker is modified to serve as initiator for the growth of the polymer segments, in a two-step polymerization. The copolymer is efficiently cleaved upon UV irradiation, whether in solvent or in aqueous solution, yielding two separate polymer segments.

When hydrated, PMCL-ONB-PAA self-assemble into well defined structures, including polymersomes. They are shown to respond in a controlled manner to UV light, via a change in size and morphology. Indeed, loaded polymersomes disintegrate upon UV irradiation, yielding small micellar-like structures, and simultaneously releasing their payload. The versatility of our 
system is tested both for small molecular weight molecules (Fluorescein and ATTO655 dye), and for proteins (enhanced green fluorescent protein). By varying the UV intensity, the payload is released in a controlled manner, as established by fluorescence spectroscopy and fluorescence correlation spectroscopy. Therefore, these responsive polymer vesicles serve as smart triggerable nanocarriers that can be applied for a variety of payloads, ranging from conventional drug molecules to proteins, enzymes, or DNA. 


\section{Contents}

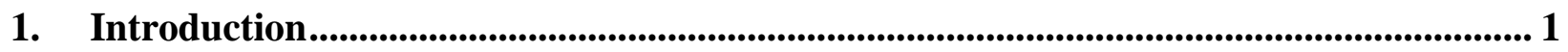

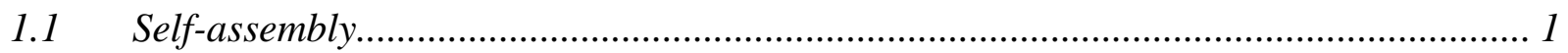

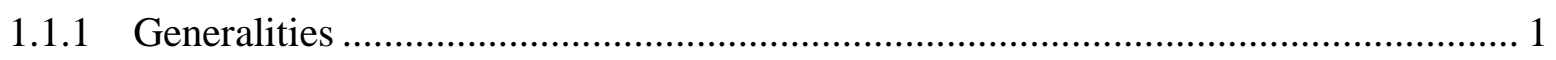

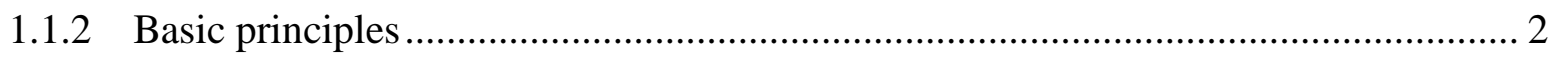

1.1.3 Theory on self-assembly process with block copolymers ........................................ 4

1.1.4 Types of structures: micelles and vesicles ......................................................... 8

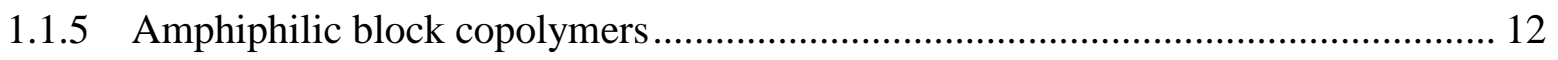

1.2 Smart materials for drug delivery applications …………..................................... 14

1.2.1 Stimuli-responsive self-assemblies ................................................................... 15

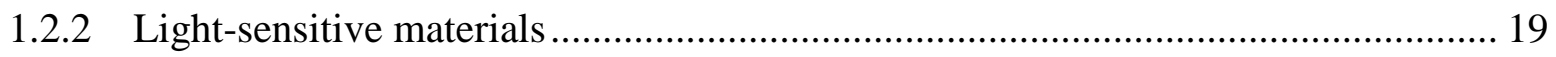

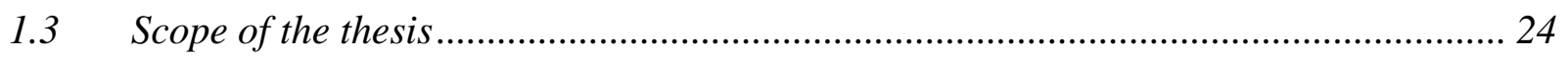

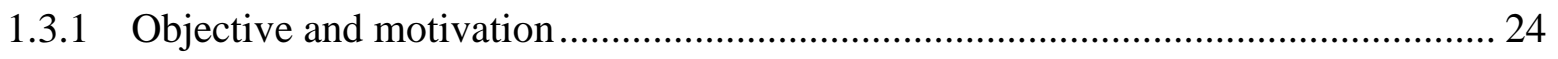

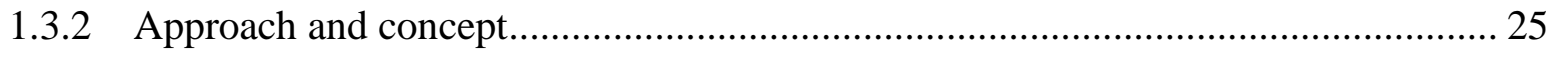

2. Synthesis of new photocleavable block copolymers.................................................................. 29

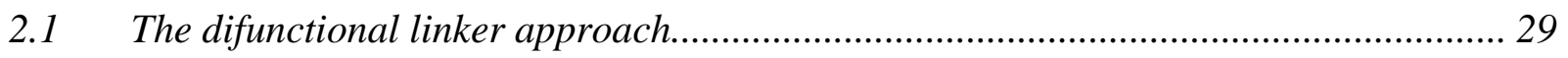

2.2 Synthesis of a new photocleavable moiety .................................................................... 31

2.3 PMCL-ONB-PAA diblock synthesis....................................................................... 34

2.3.1 ROP of $\varepsilon$-caprolactone and $\gamma$-methyl- $\varepsilon$-caprolactone monomers initiated by ONB. 34

2.3.2 ATRP of tBA with PMCL-ONB macroinitiator ....................................................... 39

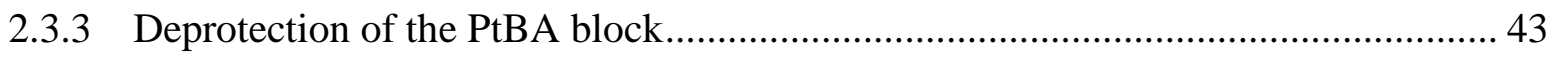

3. Photodegradation of polymer chains ........................................................................................... 46 


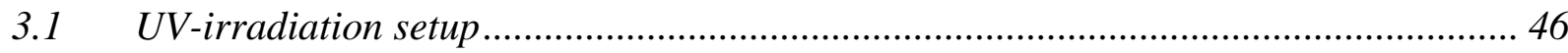

3.2 Block copolymer photocleavage in solvent t................................................................ 47

3.2.1 ONB photodegradation characterized by UV-Vis spectroscopy .............................. 48

3.2.2 ONB photodegradation followed by ${ }^{1} \mathrm{H}-\mathrm{NMR}$ spectroscopy …….......................... 50

3.2.3 GPC analysis: evidence of block cleavage ……………………………………..... 51

3.3 Block copolymer photocleavage in water ……………………................................ 53

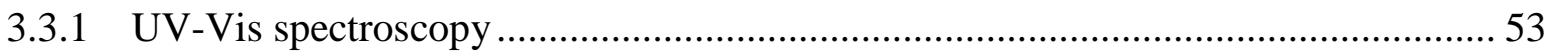

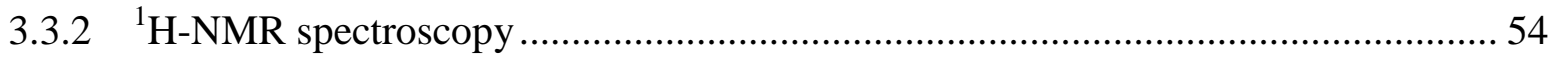

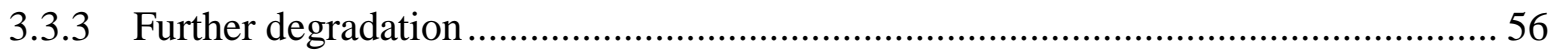

4. Self-assembled structures and their UV-triggered degradation....................................... 60

4.1 Self-assembly of PMCL-ONB-PAA and PCL-ONB-PAA copolymers in PBS .............. 60

4.1.1 Preliminary studies with PCL-ONB-PAA and PMCL-ONB-PAA ........................... 60

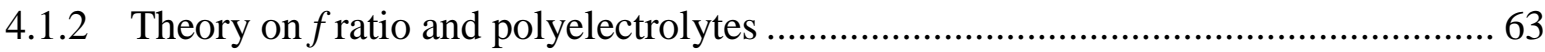

4.1.3 Polymers with low $f$ ratios forming vesicles............................................................. 64

4.2 Photodegradation of self assembled structures ........................................................... 68

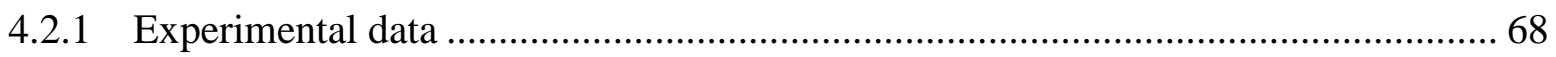

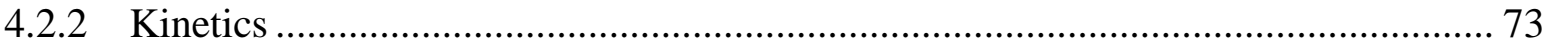

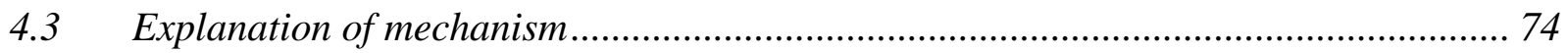

4.3.1 Common degradation mechanism for responsive polymersomes ........................... 75

4.3.2 Proposed mechanism for our system …………................................................... 76

5. Encapsulation and release of hydrophilic molecules ............................................................. 80

5.1 Encapsulation of low molecular weight molecules....................................................... 80

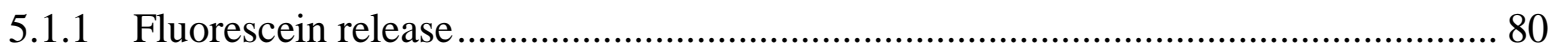


5.1.2 Release of ATTO655 followed by fluorescence correlation spectroscopy (FCS).... 82

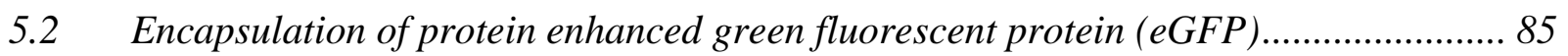

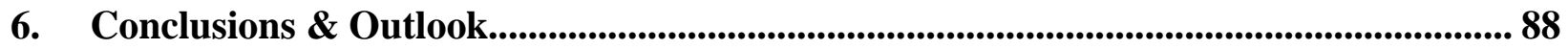

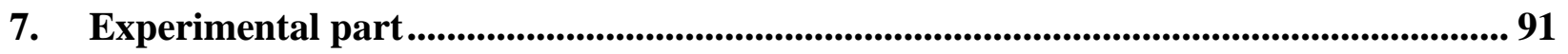

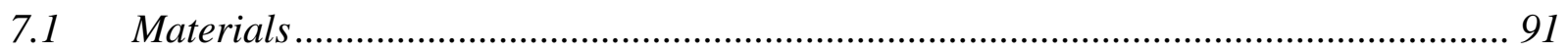

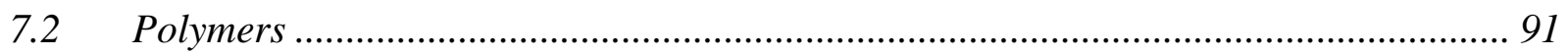

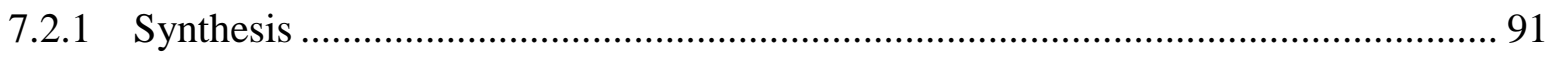

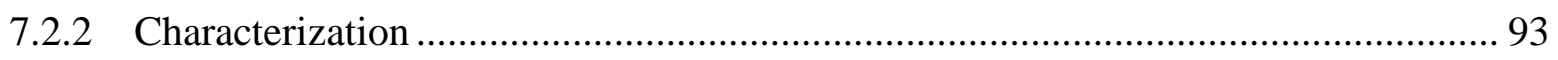

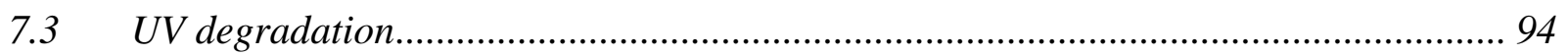

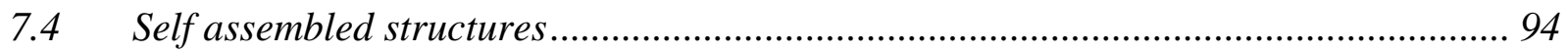

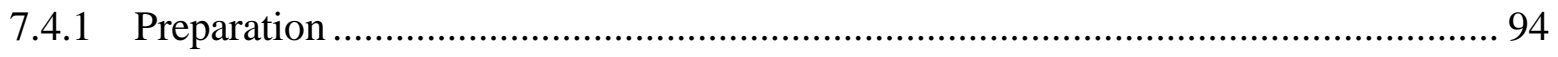

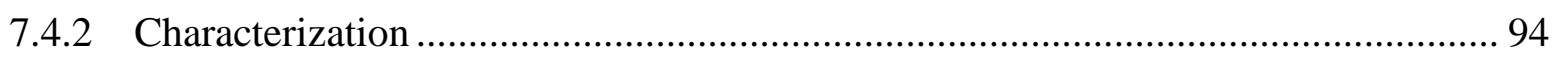

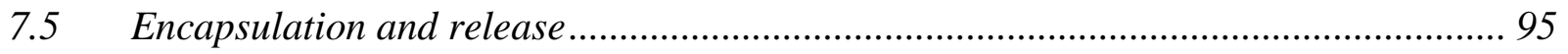

7.5.1 Preparation of loaded vesicles ................................................................ 95

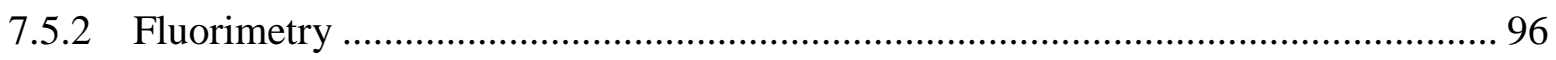

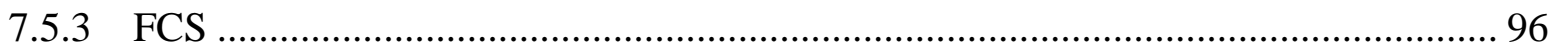

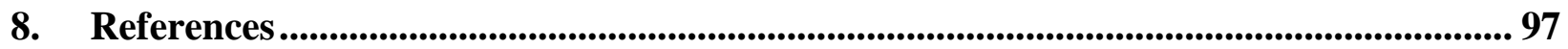

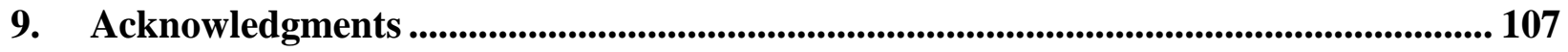

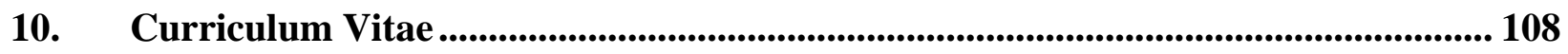




\section{Introduction}

The goal of this thesis was to design photoresponsive polymeric vesicles via the selfassembly of photocleavable amphiphilic block copolymers, and to prove their potential use as nanocarriers for therapeutic molecules and proteins.

In this introduction, the basics to understand self-assembly are reviewed, and relevant examples of stimuli-responsive vesicles are discussed.

\subsection{Self-assembly}

\subsubsection{Generalities}

Polymeric vesicles, also coined as polymersomes, are a class of smart materials obtained from a bottom-up approach, involving the spontaneous organization of macromolecular building blocks (amphiphilic synthetic block copolymers in our case) into a well defined structure. Those building blocks may have functionalities at macromolecular level on their own, but when selfassembled, they become part of a whole new structure, typically in the nanometer size range, and they convey completely different properties to it.

For example, in nature, the cooperative interactions between small building blocks (lipids, polypeptides, polysaccharides) give rise to the formation of essential organelles and bioactive compounds, such as cell membranes and proteins. In the field of nanotechnology, such self-assembled systems are already in use to address new challenges in biomedicine (nanoreactors, nanocarriers, biosensors, etc...), microelectronics (templated surfaces for crystal growth, microstrucutured materials with molecular switches, etc...).

The understanding and control of self-assembly process (i.e. understanding structure/property relationships) is essential to the discovery of new systems with unique properties, and will enable the rational design of molecular systems in order to optimize their performances. It is already known that the spontaneous organization of macromolecular building 
blocks is due to a combination of forces such as hydrophobic interactions, Van der Walls forces, and hydrophilic interactions.

In order to mimic nature and fabricate well-defined self-assembled structures, one has to carefully design building blocks with features allowing such interactions. In this regard, synthetic block copolymers represent a very interesting class of materials. They can self-assemble to form a variety of superstructures. For example, in the diluted regime, block copolymers can selforganize into very simple aggregates known as micelles. According to different parameters, imparted by a diversity of polymer chemistries, architectures, or lengths, they can also form structures such as cylindrical micelles and vesicles.

Therefore, with these synthetic polymers, we can do more than mimicking nature: by introducing specific functionalities to the polymer, we can direct self-assembly toward desired morphologies, or confer other properties on demand, such as responsiveness, to new systems.

\subsubsection{Basic principles}

As described by Forster et al. ${ }^{[1]}$ the first prerequisite for synthetic block copolymers to be used in self-assembly, is to be constituted of two polymer segments having both long-range repulsive, and short-range attractive forces. In other words, for block copolymers, they must be constituted of at least two covalently linked (short-range attractive) segments with different polarities (long-range repulsive) (Figure 1). Such structures are called amphipathic, or amphiphilic (in the case of water) and resemble more conventional molecules such as surfactants or lipids. 


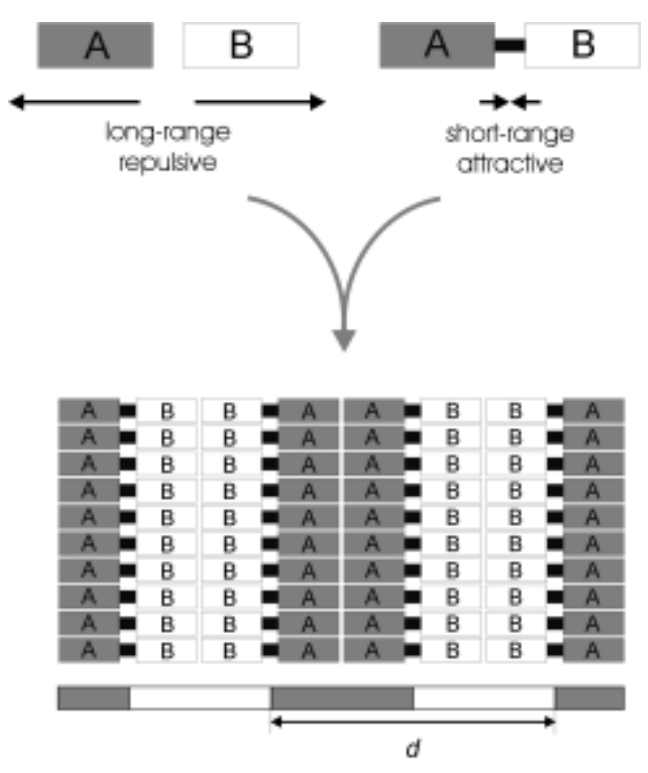

croered structure

Figure 1: Illustration of long-range repulsive and short-range attractive forces leading to an ordered structure by self-organization (d is the periodic length of the structure). ${ }^{[1]}$

The basic principle of self-assembly is based on the poor solubility of one of the blocks in a given solvent, while the second block has good solvent-solute interactions. In the case of water as solvent, although attractive forces in between hydrophobic chains are weak, they will pack to segregate out of the aqueous environment, this both due to weak interactions with the water molecules, and to the large energy barrier that cause the disturbance of the water lattice surrounding them. ${ }^{[2]}$ In contrast, the hydrophilic chains are more soluble in water, due to strong solvent-solute interactions.

Intuitively, one can deduce from this basic principle the most simple self-assembled structure induced by such forces, the micelle. In those self-assembled structures, the hydrophobic chains aggregate into a core to avoid contact with solvent molecules, while the hydrophilic chains interacting with solvent molecules form a corona at the surface. It should be noted that this example is only valid in the diluted state, where the critical micellar concentration (CMC) is extremely low for amphiphilic block copolymers when compared to low molecular weight 
surfactants. In this thesis, the behavior of the synthesized block copolymers was only studied in the diluted regime, where structures like micelles and polymersomes can be found.

In the next paragraph, we give a short theoretical background on the formation of selfassembled structures generated by synthetic amphiphilic block copolymers in water.

\subsubsection{Theory on self-assembly process with block copolymers}

As we explained briefly, hydrated amphiphilic block copolymers behave as surfactants and lipids and they can self-assemble to form sophisticated supramolecular structures, such as spherical micelles, rods, or nanotubes, and vesicles in diluted solutions, but also many others in lyotropic and bulk phases (Figure 2). ${ }^{[3-5]}$

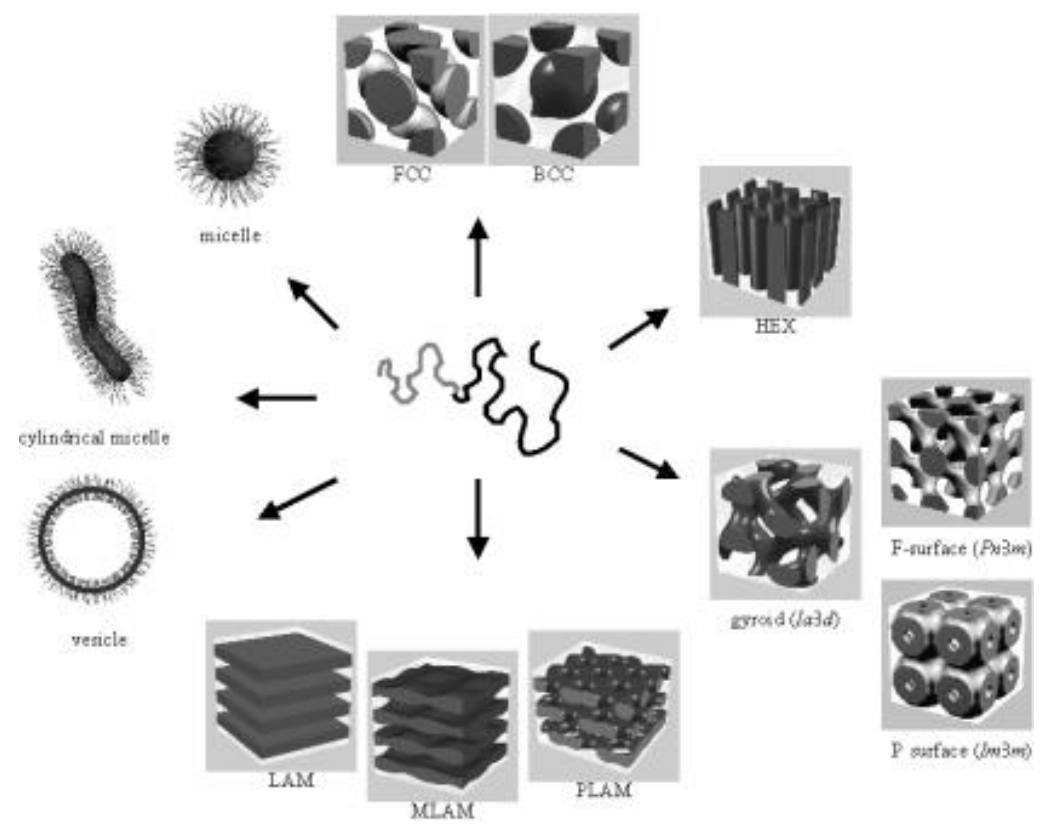

Figure 2: Examples of superstructures obtained with amphiphilic block copolymers. ${ }^{[1]}$

There is an ongoing debate as to know whether the aggregates formed upon selfassembly are kinetically frozen or equilibrium structures (thermodynamic). As of today, due to 
the incredible diversity in block copolymers, there is no universal theory able to predict which morphology will be adopted preferentially. However, several key parameters are known to influence the self-assembly and will be briefly reviewed here.

Self-assembly of amphiphilic block copolymers has been described in terms of geometric constraints, directly related to the macromolecular features of the polymer chains, and thermodynamic considerations, such as minimization of the total free energy of the system, involving the decrease of interfacial tension at hydrophilic/hydrophobic interface and the entropy loss from polymer chains. ${ }^{[6]}$

\subsubsection{Geometric constraints}

From a geometry perspective, the morphology is best described using the packing parameter $p$ (Equation 1). ${ }^{[7]}$

$$
p=\frac{v}{a l}
$$

Equation 1

This parameter encompasses the volume of the hydrophobic block $(v)$, the area covered by hydrophilic groups $(a)$, and the length of the hydrophobic block $(l)$. $p$ can also be related to the radius of curvature through Equation 2:

$$
p=1-H l+\frac{K l^{2}}{3}=1-\frac{1}{2}\left(\frac{1}{R_{1}}+\frac{1}{R_{2}}\right)+\frac{l^{2}}{3 R_{1} R_{2}}
$$

where $K$ is the Gaussian curvature and $H$ is the mean curvature, $R_{1}$ and $R_{2}$ are curvature radii. ${ }^{[6]}$ As can be deduced from Equation 2, $p$ approaches unity for very large curvature radii, which is characteristic of vesicular shapes. Such a high curvature is the result of the preferential chain packing upon bilayer formation, driven by volume and steric constraints. As an example, this phenomenon was illustrated by Discher et al. for poly(ethylene oxide)-b-poly(butadiene) (PEOPBD) aggregates. ${ }^{[8]}$ In their work, they also define a convenient quantity, the hydrophilic to hydrophobic ratio $f$ to characterize this phenomenon. As a general rule, copolymers with ratios 
above 0.5 tend to form preferentially micelles, when copolymers with ratios less than 0.33 tend to form vesicles (Figure 3). Although this ratio gives a good approximation, it may not be applicable to all systems.

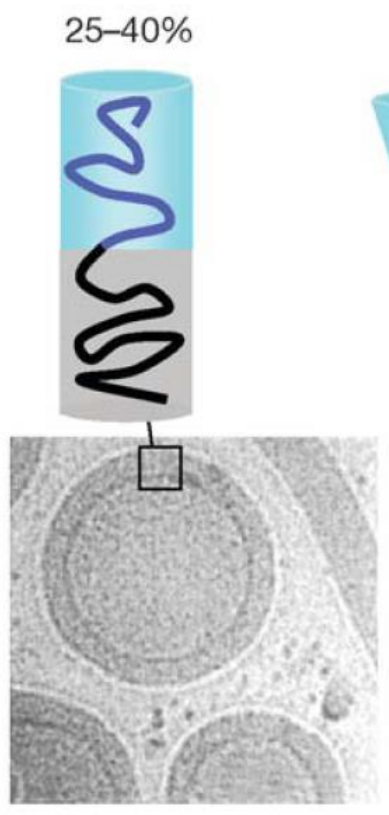

$40-50 \%$

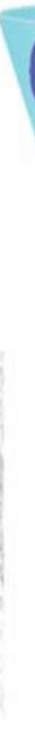

$>50 \%$

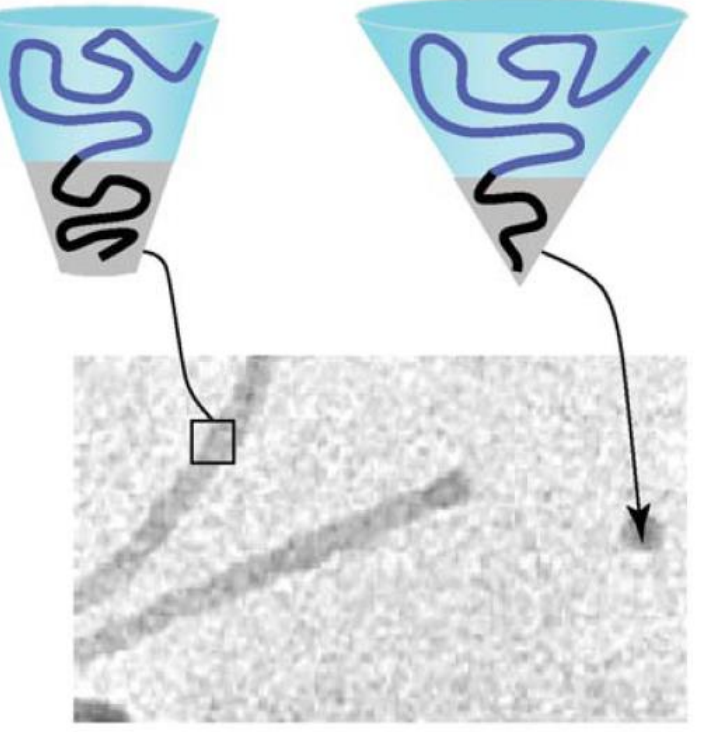

Figure 3: Schematics of block copolymer fractions with respective cryogenic transmission electron microscopy images showing vesicles or worm micelles and spherical micelles associated with different $f$ ratios. ${ }^{[8]}$

\subsubsection{Thermodynamics of vesicle formation}

The geometric elements are not sufficient on their own to describe the self-assembly of amphiphilic copolymers. It appears that in many systems, thermodynamics may have a significant role. In this model, a thermodynamic equilibrium is reached via minimization of the system's total free energy. It involves the decrease of the interfacial energy, and the loss of entropy from polymer chains upon aggregation in small domains. According to the nature of the polymer chains, association thermodynamics will be dominated by one or the other contributions. 
The interfacial area per unit volume, $A_{v}$, is given by Equation 3:

$$
A_{V}=d \varphi / l
$$

Equation 3

where $d$ is the dimensionality, $\varphi$ is the volume fraction of hydrophobic domain, and $l$ is the chain length normal to the interface.

To decrease the interfacial energy, amphiphilic block copolymers will self-assemble by minimizing the interfacial area. According to Equation 3, the lowest interfacial area is obtained for a dimensionality of 1 , thus favoring flat bilayer structures. According to Antonietti and Förster, ${ }^{[6]}$ such flat bilayers can then close up to form vesicles, in a process called vesiculation described in Figure 4.
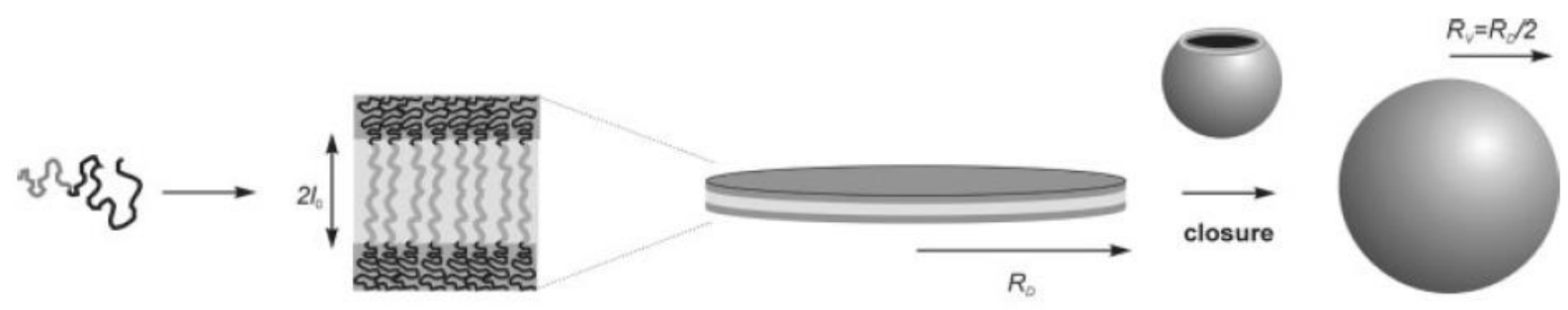

Figure 4: Vesiculation process, showing the closure of flat bilayers to vesicles. ${ }^{[6]}$

The second contribution to the decrease of the total free energy, results from the entropic loss induced by segregation of polymer chains upon self-assembly. The intrinsic nature of synthetic polymers implies that there is a certain distribution of molecular weights (polydispersity), which can in turn play a significant role in the self assembly. ${ }^{[6,9]}$ As was shown by Terreau et al. for a series of poly(styrene)-b-poly(acrylic acid) (PS-PAA) copolymers, shorter hydrophilic chains are segregating toward the inner part of the membrane, were the area available for hydrophilic groups is smaller, thus favoring changes in curvature. ${ }^{[10]}$ The extent of entropic loss of a polymer chain is closely related to the internal degree of freedom. ${ }^{[6]}$ Hence, for 
stiff polymers with low flexibility, the decrease in free energy will be dominated by the minimization of interfacial area rather than entropy loss.

\subsubsection{Types of structures: micelles and vesicles}

In this part, polymeric micelles and vesicles will be discussed in detail, as they are the main structures encountered in this thesis.

\subsubsection{Micelles}

Micelles have a hydrophobic core constituted by hydrophobic segments (A), and a hydrophilic corona constituted of water-soluble segments (B) (Figure 5).

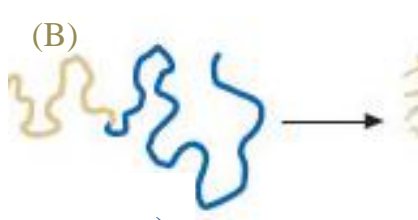

(A)

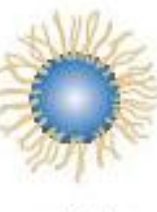

micelle

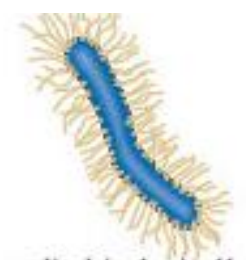

cylindrical micelle

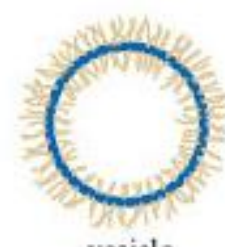

vesicle

Figure 5: Examples of the different self-assembled structures adopted by block copolymers with hydrophobic $(A)$ and hydrophilic $(B)$ elements in the diluted regime.

Therefore, the size of micelles is well-defined, by the length of the polymer blocks. They cannot be larger (in diameter) than twice the length of an AB diblock, assuming that the polymer chains are fully stretched both in the corona and in the core. In this regard, the nature of polymer segments plays an important role. For instance, corona-forming charged polymers exert a pulling force on core-forming chains. As stated by Meier and coworkers, ${ }^{[11]}$ the flexibility of the chains is also an important parameter: polymers with low glass transition temperatures $\left(\mathrm{T}_{\mathrm{g}}\right)$ (polybutadiene for instance) can easily adopt new conformations in contrast to stiff chains with high $\mathrm{T}_{\mathrm{g}}$ (such as polystyrene). 
In terms of shape, the morphology of micelles is mostly influenced by the hydrophilic block architecture. According to the model used by Antonietti and Forster, ${ }^{[6]}$ the shape of one building block is directly correlated to the curvature of the aggregate. This is maybe best described by the hydrophilic to hydrophobic ratio $f$ that was discussed above.

The hydrophobic core of micelles can solubilize a number of hydrophobic dyes and drugs. But one important limitation of micelles is that they are not designed to carry hydrophilic payloads, since a number of therapeutic molecules are hydrophilic, and need to be encapsulated into structures comprising a hydrophilic compartment.

As was proposed by Kita-Tocarczyk et al. ${ }^{[11]}$ micelles may be seen as a crude example of a bilayer structure, only they do not have two sides exposed to water, and therefore, they do not comprise an inner pool. In the next paragraph, such vesicular structures are discussed.

\subsubsection{Vesicles}

\subsection{Polymersome formation}

In addition to the vesiculation process for vesicle's formation proposed by Antonietti et $a l .^{[6]}$ (two-step process where bilayer close up to form vesicles), two other mechanism have been proposed for the formation of vesicles. Those mechanisms where developed with theoretical calculations, ${ }^{[12]}$ and latter experimentally observed. In both mechanisms, block copolymers initially self-assemble into small spherical micelles. In mechanism I (Figure 6), those micelles evolve toward larger micelles with disc-like or elongated shapes occurring via collision. Eventually, the disc-like micelles then close up to form polymersomes. Mechanism I was nicely illustrated by the experiments performed with a poly(ethylene oxide)-b-poly( 3-(trimethoxysilyl) propyl methacrylate) (PEO-b-PTMSPMA) block copolymer. ${ }^{[13]}$ Transition states induced by gradual solvent exchange (from methanol to water) were trapped and observed by TEM. In the methanol rich solution, a majority of micelles was observed, which slowly evolved to rod-like micelles, and finally to vesicular morphology. 


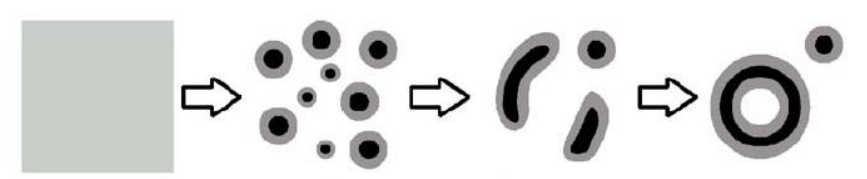

(a) Mechanism I

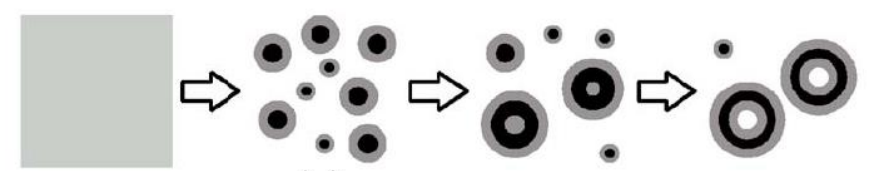

(b) Mechanism II

Figure 6: Schematic representation of vesicle formation with mechanism I and II, from the initial homogeneous state (grey squares). Grey parts represent hydrophilic blocks, while black parts represent hydrophobic parts. ${ }^{[12]}$

In the second mechanism, polymer chains initially not involved in a micelle slowly aggregate with the existing micelles, increasing their size. When the energy cost associated with the bending, solvent diffused inside these oversized micelles to increase the curvature radius and finally yield vesicular structures. Adams et al. studied the impact of mechanism of formation on encapsulation in block copolymer vesicles, using a poly(ethylene oxide)-bpoly(diethylaminoethyl methacrylate) (PEO-b-PDEAMA). ${ }^{[14]}$ In their work, they report extremely low encapsulation efficiency which can be best explained by mechanism II. In contrast with the first mechanism, where the large micelle close up and therefore imprison water in the inner pool, in the second mechanism micelles slowly transform to vesicle without transiting by an "open system", preventing solute molecules to get inside the inner pool.

\subsection{Polymersome size}

It is known that polymersomes made from block copolymers are often trapped in a nonequilibrium structure, or kinetically frozen structure, rather than a thermodynamic equilibrium. ${ }^{[15]}$ The mobility of polymer chains within a bilayer or from one self-assembled structure is extremely slow. Therefore, the size of vesicles is strongly dependent on the fabrication process, and can be tuned by changing preparation conditions. Generally, vesicle can be prepared with two main techniques, the co-solvent method where the AB copolymers are 
dissolved in a good solvent for both blocks, slowly exchanged with water, and a solvent-free method, where the polymer is directly dissolved in water. In the case of the co-solvent method, which was used in this study, parameters such as order of solvent addition, addition duration, and affinity of solvent with water, play an important role over control of vesicle size.

As opposed to micelles, for which size is limited to the nanometer range, vesicle sizes can be found anywhere in between 100nm and several micrometers in diameter. The size range is particularly important for in vivo applications: it is well known that the size of carriers is a key feature in the design of an ideal drug delivery system (DDS), since it is directly linked to its blood clearance and circulation time. ${ }^{[16,17]}$ Therefore, although micrometer-size particles are interesting from a practical point of view (easily characterized with imaging techniques) and have high loading capacity, nanometer-size polymersomes are seen as better candidates for drug delivery applications.

\subsection{Polymersome membrane properties}

Structurally, polymer vesicles are very similar to liposomes: they are hollow spheres with an aqueous core, separated from the outside medium by membranes. However, the synthetic structures present features which outperform liposomes, because their membranes have special properties.

Liposome membranes formed by lipids with well-defined molecular weights, are relatively thin $(3-5 \mathrm{~nm})$, fluid, and have poor mechanical stability. They are permeable to low molecular weight compounds and poorly resistant to lysis strain. The reinforcement of liposome membranes with PEGylation or introduction of cholesterol was explored, but it did not drastically improve their stability. In contrast, synthetic polymers form viscous and robust membranes that are thicker and far more stable than liposome membranes. ${ }^{[18,19]}$

The thickness of the membrane was shown to be dependent on the molecular weight of the hydrophobic block, with different scaling laws according to the polymer system studied. The power law $\mathrm{d} \sim \mathrm{M}_{\mathrm{w}}{ }^{\mathrm{b}}$ relates the molecular weight $\left(\mathrm{M}_{\mathrm{w}}\right)$ to the thickness $\mathrm{d}^{\left[{ }^{[20]}\right.}$ Typically, thicknesses ranging from a few nanometers up to $40 \mathrm{~nm}$ may be obtained. ${ }^{[21]}$ The control over thickness via 
the hydrophobic block length is an important advantage over liposomes since the membrane basically defines the physical properties of polymeric vesicles.

The global stability of polymer vesicles also results from a specific attribute that only block copolymers have: polydispersity. As stated before, it has been shown that it allows chain segregation, with short hydrophilic chains toward the inside and higher molecular weight chains toward the outside, thus favoring curvature and stabilization of bended bilayers. Long-term stability is also linked to the very slow chain exchange dynamics of polymer chains, implying that polymer vesicles are not prone to dissociation.

Another important characteristic of polymer chains is flexibility. Due to their greater lengths, block copolymers can undergo a lot more conformational arrangements than lipids. This in turn facilitates their packing into membranes, where they may adopt different conformations: interdigitated (which represents best the real nature of most vesicle membranes), stretched or collapsed (which represents theoretical boundaries). ${ }^{[22]}$ This can confer increased toughness and lower permeability to the membrane, especially when compared to lipidic membranes which are considered to be leaky.

The different advantages of polymeric vesicles conferred by their outstanding membranes make them ideal materials to be use as drug or gene vehicles.

\subsubsection{Amphiphilic block copolymers}

In addition to the structural or mechanical advantages they bring to membranes, synthetic block copolymers provide a versatile portfolio of building blocks, with the possibility to introduce functionality into self-assembled structures.

Amphiphilic block copolymers can be synthesized with a variety of polymerization techniques and coupling chemistries, which allows the fabrication of macromolecules with diverse architectures (including diblocks ${ }^{[19,21,23,24]}$ and triblocks, ${ }^{[25-27]}$ but also dendritic ${ }^{[28-30]}$ and graft $^{[31]}$ copolymers), chemistries, compositions, and lengths (Figure 7). As we have seen before, all these characteristics may be used to control several parameters of the self-assembly process. 


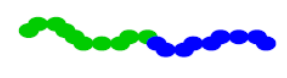

AB diblock

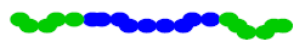

ABA triblock
Cyclic AB diblock

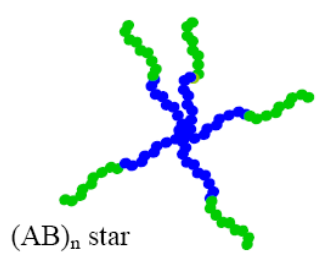

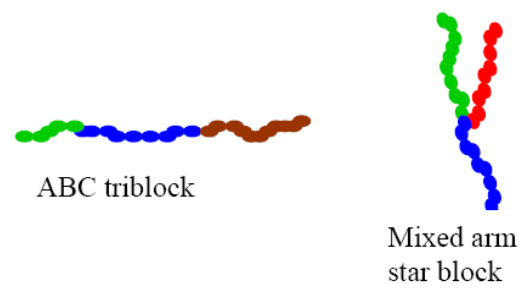

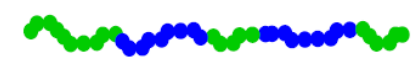

$(\mathrm{AB})_{\mathrm{n}}$ multiblock

Figure 7: Examples of block copolymer architectures. ${ }^{[32]}$

Living anionic and cationic polymerizations, and living radical polymerization are among the most advanced techniques used to produce $\mathrm{AB}$ block copolymers. Using those polymerizations, a large number of strategies for preparing block copolymers exist (they are presented in the first chapter). In this thesis, we combined two living polymerizations (ring opening polymerization and atom transfer radical polymerization) to produce our block copolymers. In this approach, the use of multifunctional initiators opens up possibilities to combine mechanistically incompatible polymerizations, and widens the choice of monomers.

Indeed, an exhaustive list of vesicle-forming $\mathrm{AB}$ block copolymers cannot be given in this thesis. To get an idea of the diverse copolymers forming polymersomes, the reader is invited to read the review from LoPresti et al. in which a summary table is given as supporting information. ${ }^{[22]}$

A short overview of self-assembled structures obtained with copolymers comprising one of the blocks we used in our block copolymer is given. It should be noted that an exactly similar block as never been produced before.

A number of studies report on the self-assembly of PAA containing amphiphilic block copolymers. Eisenberg et al. first reported multiple morphologies of aggregates of polystyrene- $b$ - 
poly(acrylic acid) copolymers, including rods, spheres, and vesicles, ${ }^{[10,24]}$ as well as vesicles from a poly(ethylene oxide)- $b$-poly(caprolactone)-b-poly(acrylic acid) triblock copolymer. ${ }^{[26]}$ According to $\mathrm{Yu}$ et al., polybutadiene- $b$-poly(acrylic acid) copolymers can self assemble into different aggregates, including vesicles. ${ }^{[33]}$ With other systems, such as poly(butyl acrylate)- $b$ poly(acrylic acid), ${ }^{[34,35]}$ poly(caprolactone)- $b$-poly(acrylic acid), ${ }^{[36]}$ poly(2-cinnamoylethyl methacrylate)- $b$ - poly(acrylic acid), ${ }^{[37]}$ poly(isoprene)- $b$-poly(acrylic acid), ${ }^{[38]}$ and poly(acrylic acid)-b-poly(methyl acrylate), ${ }^{[39]}$ micellar structures were obtained.

Only one example of PMCL containing block copolymer forming vesicles was reported. ${ }^{[40,41]}$ In our group, Braun showed that vesicles of PEG-PMCL were easily formed under specific conditions. ${ }^{[40]}$ According to his work, the weight ratio of hydrophilic to hydrophobic $(f)$ is a key parameter to drive the morphology of the aggregates toward vesicular like structures.

\subsection{Smart materials for drug delivery applications}

As mentioned above, synthetic block copolymers are not only good alternatives to surfactants or lipids as amphiphilic building blocks, but they are also superior in terms of functionality.

According to Onaca et al.$^{[18]}$ intelligent drug delivery systems require the following basic features: "adequate stability in the bloodstream, long circulation properties, and selective accumulation at the site of action together with a suitable drug release profile and good biocompatibility".

In this thesis the work is limited to the design of a responsive nanocarrier (that is to fulfill the requirement of suitable release profile). As described above, the introduction of new functionalities and a variety of possible chemistries in amphiphilic block copolymers, provide a whole new range of properties, in addition to their self-assembling properties. In this respect, it is possible to introduce chemically active monomers or biologically active moieties to induce respectively responsiveness and targetability to those polymersomes. 
The mechanical advantage offered by AB block copolymers over lipids may also severely limit the applications of such nanocarriers: the improved stability is also preventing small molecules to escape the inner core of such structures via diffusion through the polymer membrane. ${ }^{[42]}$ Therefore, in self-assembled systems used as nanocarriers, a crucial point is to look for different approaches to modify the permeability of polymersome membranes, in order to promote the release of entrapped compounds. The most successful and elegant answer to this problem, is the use of stimuli-sensitive copolymers to fabricate responsive nanocarriers.

\subsubsection{Stimuli-responsive self-assemblies}

The underlying strategy behind such system is based on a dramatic physicochemical change caused by the stimuli. At macromolecular level, polymer chains can be altered in different ways, including changes in hydrophilic to hydrophobic balance, conformation, solubility, degradation, or bond cleavage which will, in turn, cause considerable behavioral changes to self-assembled structures. ${ }^{[43]}$ Many designs with the location of responsive moieties or functional groups are possible. The locations include, but are not limited to: side chains on one of the blocks, chain end-groups, or junction points in between blocks. The response may be reversible or not depending on the strategy employed. The tailored properties of polymersomes build from those polymers make them suitable in medical applications, acting as smart systems. As a result, the area of drug nanocarriers is currently the most active and exciting area of development in the field of controlled DDS.

Stimuli are commonly classified in three categories, of physical, chemical, or biological nature. ${ }^{[44,45]}$ Physical stimuli (light, temperature, ultrasounds, magnetic, mechanical, electric) usually modify chain dynamics, i.e. the energy level of the polymer/solvent system, while chemical stimuli (solvent, ionic strength, electrochemical, $\mathrm{pH}$ ) modulate molecular interactions, whether in between polymer and solvent molecules, or in between polymer chains. ${ }^{[46]}$ Biological stimuli (enzymes, receptors) can be related to functions of molecules: enzyme corresponding chemical reactions, receptor-recognition of molecules. ${ }^{[45]}$ The next sub-sections only highlight the most promising stimuli, $\mathrm{pH}$, temperature, and redox. Light responsive systems are described in detail in a specific subchapter. 


\subsubsection{1 pH-responsive systems}

In general, the $\mathrm{pH}$ responsiveness of a polymer is obtained via protonation or deprotonation of a polybase or polyacid, $\mathrm{pH}$-induced conformational changes, or selective cleavage of $\mathrm{pH}$ sensitive bonds.

The first $\mathrm{pH}$ sensitive vesicles made of polystyrene-b-poly(acrylic acid) were reported by Eisenberg et al. in $1995 .{ }^{[24]}$ Recently, his group reported on vesicles formed by a triblock poly(acrylic acid)- $b$-poly(styrene)- $b$-poly(4-vinyl pyridine) (PAA- $b$-PS- $b$-P4VP), undergoing a reversible pH-triggered inversion of the membrane. ${ }^{[4]}$ The switch is based on the protonation/deprotonation cycle of the two amphiphilic blocks: at $\mathrm{pH} \mathrm{1,} \mathrm{P4VP} \mathrm{chains} \mathrm{are}$ positively charged, water soluble, and located on the outside corona, while PAA chains are protonated, not water soluble, and located inside. At $\mathrm{pH}$ 14, the PAA chains are negatively charged, the P4VP chains are deprotonated and insoluble, and the vesicles have an inverted membrane: PAA outside, P4VP inside.

Another example of $\mathrm{pH}$ sensitive vesicle was proposed by Armes and coworkers. ${ }^{[48]}$ They developed a highly biocompatible and $\mathrm{pH}$-sensitive block copolymer, PDPA- $b$-PMPC. The PDPA block is deprotonated at $\mathrm{pH}$ above 7 (pKa around 5.8-6.6), and therefore insoluble. DNA and water-soluble Doxorubicin were encapsulated within PDPA-b-PMPC vesicles, and released upon lowering of the solution $\mathrm{pH}$.

Using polypeptide based block copolymers, several groups reported on the successful formation of $\mathrm{pH}$-responsive polymersomes, usually triggered by a conformational change of one of the blocks. As an example, Lecommandoux and Rodriguez-Hernandez prepared vesicles with poly(L-glutamic acid)-b-poly(L-lysine) copolypeptides (PGA-PLys). ${ }^{[49]} \mathrm{At} \mathrm{pH}<4$, the polybase is in a coil conformation, while the polyacid is protonated and the hydrogen bonds induce a conformational change from a coil structure to an $\alpha$-helical structure. The PGA block is then water insoluble, and forms the hydrophobic part of the membrane. At $\mathrm{pH}>10$, the structure is inverted, with $\alpha$-helices of PLys forming the core of the membrane, and PGA charged chains on the outside. 
Recently, an example of block copolymer bearing a $\mathrm{pH}$-sensitive linkage in between hydrophilic and hydrophobic segments was reported. The PDMAEMA and PEG blocks are connected via an ortho-ester, which can be cleaved upon $\mathrm{pH}$-trigger. ${ }^{[50]}$

\subsubsection{Temperature responsive systems}

Nearly all temperature sensitive systems are based poly(N-isopropylacrylamide) (PNIPAM), a well known thermo-responsive polymer. This polymer has a lower critical solution temperature (LCST) of $32^{\circ} \mathrm{C}$. Below this temperature, it is molecularly dissolved in water, and above $32^{\circ} \mathrm{C}$, the hydrophilicity changes.

A number of studies with block copolymers combining PNIPAM with another hydrophilic polymer block, reported on temperature-sensitive vesicles. For example, Li et al. prepared vesicles with a poly([N-(3-aminopropyl)-methacrylamide hydrochloride]-b-poly(Nisopropylacrylamide) PAMPA-PNIPAM block copolymer. ${ }^{[1]}$ Above the LCST of PNIPAM, vesicles spontaneously form with PNIPAM forming the membrane core, and PAMPA as the hydrated corona. The transition from fully dissolved diblocks to self-assembled structures is reversible, unless ionic crosslinking locked the vesicular structures.

Using a similar block copolymer, PEO-PNIPAM, Qin et al. ${ }^{[52]}$ prepared vesicles which can encapsulate Doxorubicin, and sequester a hydrophobic dye in their membranes. Upon cooling to temperatures below $32^{\circ} \mathrm{C}$, the membrane is dissolved, and both contents are released upon complete dissociation of the vesicles.

It is also interesting to mention that polypeptide based block copolymers may also show temperature induced conformal changes, from $\alpha$-helical to $\beta$-sheets structures.

\subsubsection{Redox responsive systems}

Hubbell and coworkers ${ }^{[53]}$ developed triblock copolymers PEO-PPS-PEO (PPS stands for polypropylene sulfide), and showed that upon exposure to oxidative agents such as $\mathrm{H}_{2} \mathrm{O}_{2}$, the middle hydrophobic block was oxidized to hydrophilic polypropylene sulfoxide and 
polypropylene sulfone, thereby achieving a gradual transition from amphiphilic triblocks to fully hydrophilic chains. Polymersomes were dissociated into individual polymer chains within hours.

The same group later reported on a reduction sensitive block copolymer. In this case, they produced a PEO-SS-PPS diblock copolymer with a reduction sensitive disulfide bond placed in between the two polymer segments. ${ }^{[54]}$ These copolymers spontaneously self-assemble into vesicular structures, which can be rapidly disrupted in the presence of cysteine in intracellular concentrations. The later is responsible for the separation of the blocks within 10 minutes, subsequently triggering the destruction of the vesicles.

\subsubsection{Other stimuli}

For other examples of the different systems available, the reader can refer to several recent reviews. ${ }^{[16,18,43,44,46,55-58]}$ A summary of relevant studies is given in Table 1.

Table 1: Overview of stimuli-responsive polymersomes. ${ }^{[16]}$

\begin{tabular}{|c|c|c|c|c|}
\hline polymers & preparation method & stimulus & degradability & ref. \\
\hline PBD-PGA & basic solution & $\mathrm{pH}$ (polyacid) & no & 13 \\
\hline Carboxyl Boltorn & water & $\mathrm{pH}$ (polyacid) & yes & 16 \\
\hline HEC-g-PAA & aqueous media & $\mathrm{pH}$ (polyacid) & no & 64 \\
\hline PEG -P2VP & phase transfer from chloroform to water & $\mathrm{pH}$ (polybase) & no & 12 \\
\hline PLE-PLL & water or $\mathrm{THF} / \mathrm{H}_{2} \mathrm{O}$ & $\mathrm{pH}$ (polybase) & yes & 68 \\
\hline PARG-PLE & $\mathrm{THF} / \mathrm{H}_{2} \mathrm{O}$ & $\mathrm{pH}$ (polybase) & yes & 69 \\
\hline PMPC-PDPA & water, $\mathrm{pH}$ from 2 to $>6$ & $\mathrm{pH}$ (polybase) & no & 62,29 \\
\hline PLL-PBLG-PLL & water at neutral $\mathrm{pH}$ & $\mathrm{pH}$ (polybase) & yes & 30 \\
\hline PEG-b-P(DEA-s-TMSPMA) & THF/aqueous solution & $\mathrm{pH}$ (polybase) & no & 63 \\
\hline PGA-PLL & acid $(\mathrm{pH}<4)$ or basic $(\mathrm{pH}>10)$ media & $\mathrm{pH}$ (schizophrenic) & yes & 61 \\
\hline PEG-b-(PG2MA-IND) & neutral $\mathrm{pH}$, adjusting the $\mathrm{pH}$ to $2.0-3.5$ & $\mathrm{pH}$-triggered hydrolysis & no & 65 \\
\hline PEG-PLA, PEG-PCL & THF/water & $\mathrm{pH}$-triggered hydrolysis & yes & $8,9,26,66$ \\
\hline PAMPA-PNIPAAM & water & temperature & no & 78 \\
\hline PLA-PNIPAAM & $\mathrm{N}, \mathrm{N}$-dimethylacetamide $/ \mathrm{H}_{2} \mathrm{O}$ & temperature & no & 79 \\
\hline PCEMA-PNIPAAM & THF/water, acetone/water & temperature & no & 80 \\
\hline PEG-PNIPAAM & water & temperature & no & 10 \\
\hline HBPO-PEG & water & temperature & no & 81 \\
\hline PEG-PPS-PEG & ethanol/water & oxidation & no & 14 \\
\hline PDMS- $b$-PFS & water & oxidation & no & 83 \\
\hline PEG-SS-PPS & film rehydration & reduction & no & 87 \\
\hline PAZoMA- $b-P A A$ & dioxane/water & UV & no & 88 \\
\hline PEG-malachite green & water & UV & no & 89 \\
\hline polystyrene with DNA-binding motifs & chloroform & hydrogen bonding & no & 92 \\
\hline oppositely charged PEG- $b$ - poly(amino acid)s & aqueous solutions & electrostatic interaction & yes & 94 \\
\hline PBD-PGA with $\gamma-\mathrm{Fe}_{2} \mathrm{O}_{3}$ nanoparticles & water & magnetic field & no & 95 \\
\hline PI-PEG with $\mathrm{Fe}_{3} \mathrm{O}_{4}$ nanoparticles & water & magnetic field & no & 96 \\
\hline P2VP-PEG with $\mathrm{Fe}_{3} \mathrm{O}_{4}$ nanoparticles & water & magnetic field & no & 96 \\
\hline PLA-PEG with air & THF/water & ultrasound & yes & 99 \\
\hline
\end{tabular}


It is interesting to mention that stimuli can also be classified as external (such as light, ultrasounds or magnetic field) and internal (temperature, $\mathrm{pH}$, redox potential, or glucose level). According to Meng et al., ${ }^{[16]}$ and as shown in Table 1, pH and temperature cues are predominant, due to their potential product scale-up and cost considerations. However, as mentioned by Keller et al. ${ }^{[57]}$ and as exposed in the next paragraph, light is arguably the most desirable stimulus of all.

\subsubsection{Light-sensitive materials}

Several studies on light responsive systems have been reported, including liposomes, ${ }^{[55,}$ ${ }^{59-63]}$ micelles, ${ }^{[64-67]}$ hybrid materials, ${ }^{[68,69]}$ microcapsules, ${ }^{[70]}$ and few accounts on vesicles. ${ }^{[71-73]}$ In the drug delivery field, light is a particularly attractive factor, because it provides precise temporal (when the light source is switched on) and spatial control (where the light is directed to). ${ }^{[55,74]}$ Furthermore, light-responsive systems do not require additional substances to trigger release, and as a result, do not modify the immediate environment around the polymersome, as is the case for systems sensitive to internal stimuli, such as $\mathrm{pH}$.

A number of light-sensitive molecules have been used in these systems. In this regard, azobenzene derivatives are the most used. This chromophore responds to both near-UV and visible light: reversible isomerization between the cis and trans conformation occurs upon light irradiation. The two conformations are known to have very different surface areas. The solubility change associated with the isomerization is responsible for reversible morphological changes within the self-assembled structures. ${ }^{[67]}$ Other molecules, such as spirobenzopyran derivatives, ${ }^{[75]}$ nitrocinnamate, ${ }^{[70]}$ and O-nitrobenzyl derivatives (ONB) can also be introduced in amphiphilic copolymers to impart light-sensitivity. Depending on the sensitive molecule used, the UVinduced changes may be reversible or not.

In the next subsections, relevant studies of different light-sensitive self-assembled systems are discussed. 


\subsubsection{Photoresponsive liposomes}

A few studies exist that report the design of photo-sensitive liposomes. Chandra et al. ${ }^{[59]}$ designed photocleavable lipids, constituted of charged amino-acids (Asp, Glu, and Lys) as polar head groups linked to alkyl chains of stearyl amine (C18) as tail group by a photocleavable Onitrobenzyl linker (ONB). They incorporated those newly designed lipids into liposomes with 1,2-disteroylglycero-3-phosphocholine (DSPC) as major constituent (95\%), and encapsulated carboxyfluorescein. The release of the dye was complete after 120 minutes, with a short lag time at the beginning, indicating a two-step process: in the first step, the polar head groups are separated from the hydrophobic tail upon UV-induced cleavage of the ONB, and then it is followed by a reorganization of the lipids where the encapsulated content can be released.

Bisby et al. ${ }^{[63]}$ used the azobenzene chromophore to synthesize photochromic lipids, (1,2(4'-n-butylphenyl)azo-4'-(g-phenylbutyroyl))-glycero-3-phosphocholine, (Bis-Azo PC), and incorporated them into liposomes. They showed that the UV-induced isomerization of the azo chromophore results in changes in membrane permeability. They exploited the alteration of the membrane to release encapsulated acridine orange and doxorubicin.

Light may also serve as trigger via the photothermal conversion of energy of absorbed light. Using this concept, Troutman et al. ${ }^{[62]}$ designed thermo-responsive liposomes coated with gold, forming a plasmon resonant coating. Irradiation with IR-light (1094nm) was shown to trigger the release of model encapsulated compounds.

\subsubsection{Photoresponsive micelles}

To date, micelles are the most common photoresponsive nanocarriers. A number of studies showed that they can have interesting applications in drug delivery, to release hydrophobic drugs.

Babin et al. ${ }^{[76]}$ prepared block copolymer micelles self-assembled from a poly(ethylene oxide)-b-poly([7-(diethylamino)coumarin-4-yl]methyl methacrylate) (PEO-b-PDEACMM) block copolymer. Upon NIR absorption, the coumarin pendant groups are cleaved, converting the hydrophobic PDEACMM polymer chains to poly(methacrylic acid) (PMA) hydrophilic 
chains. The copolymer is then fully hydrophilic, leading to destruction of micellar structures, and release of Nile Red in solution.

Matyjaszewski and coworkers developed reversible micelles with light responsiveness. ${ }^{[66]}$ The copolymer comprised a PEO hydrophilic block and a spiropyran containing block named SP. The spiropyran hydrophobic units undergo a reversible isomerization in response to light, yielding merocyanine hydrophilic structures. Micelles formed from PEO- $b$-SP can be disrupted by UV irradiation, when the PEO- $b$-SP is converted to PEO- $b$-ME, a fully water soluble diblock. They used those micelles to show encapsulation, release, and partial re-encapsulation of a hydrophobic dye (coumarin 102).

Using O-nitrobenzyl, a variety of systems has been suggested. Zhao et al. have developed a block copolymer poly(ethylene oxide)-b-poly(2-nitrobenzyl methacrylate) (PEO-PNBM) where the ONB has been placed as a side chain. ${ }^{[64]}$ Upon UV irradiation, the chromophores are cleaved and the polarity balance is changed causing the micellar structures to fall apart. A similar system was reported by Zhao and coworkers, ${ }^{[77]}$ using a poly(ethylene oxide)-b-poly(ethoxytri(ethylene glycol) acrylate-co-O-nitrobenzyl acrylate) (PEO- $b$-P(TEGEA-co-NBA)) triblock copolymer. The micelles obtain from this triblock copolymer were actually responsive to both UV-trigger and temperature-trigger, due to the thermo-responsive PTEGEA block. They showed that UV-irradiation could be use to change the LCST of the triblock, in an irreversible manner, and that the system could uptake Nile Red into the hydrophobic core of the micelles, and release it on demand.

Those examples of light-triggered release from block copolymer micelles are interesting because they show that light-responsive block copolymers can be used to provide responsiveness to self-assembled structures. However, as was stated before, the use of micellar structures is limited to the encapsulation of hydrophobic drugs in the core, when the demand for carriers able to encapsulate hydrophilic compounds is growing.

\subsubsection{Other photoresponsive supramolecular structures}

Here, a few examples of hollow polymeric structures sensitive to light are presented. 
Recently, Zhang et al. ${ }^{[68]}$ described a system built from an amphiphile constituted by a PEO hydrophilic chain linked to a malachite green derivative. Malachite green is a photochromic molecule, which responds to UV-light by the release of a cyanide anion and creation of a cation. Before UV, vesicular structures are formed in solution due to hydrophobic interactions (the malachite green forms the inner part of the membrane). After UV, the ionized groups trigger membrane disruption. The vesicles could be reversibly obtained by a temperature switch, but no encapsulation and release studies are reported.

Another interesting example of light-responsive structures was reported by Yuan et al. ${ }^{[70]}$ They prepared micrometer-size microcapsules in water/oil/water emulsion via reversible photocrosslinking of colloidal polyorganosiloxane nanoparticles (with diameters around 20nm) functionalized with nitrocinnamate moieties. The inner aqueous pool could be loaded with cyclodextrin molecules, which were released upon application of UV-light within minutes, after the reversible dissociation of the crosslinked shell. This represents an interesting system, however, the W/O/W system seem to be an important drawback for in vivo applications, since the shell contains large amounts of toluene and surfactant molecules.

We can also mention here the work from Robbins et al. ${ }^{[69]}$ They designed a tertiary system of polymersome (made of PEO-PBD block copolymers), encapsulated protein (such as ferritin), and $\mathrm{PZn}_{2}$ (a porphyrin structure). They showed that the polymersome membrane were destabilized as a result of local heating generated by the harvesting of UV light improved a synergetic action of ferritin and $\mathrm{PZn}_{2}$. They also demonstrated UV-triggered release of biocytin from those micron-size vesicles over 4 hours.

\subsubsection{Photoresponsive vesicles}

As mentioned earlier, despite the fact that photoresponsive polymersomes would have several advantages over other stimuli, but also over photoresponsive lipids and micelles, only a few relevant systems have been described so far.

The first tentative block copolymers explored to form photodegradable vesicles contained azobenzene moieties. Zhao and coworkers reported the formation of vesicles with 
PAzo- $b-\mathrm{P}(t \mathrm{BA}-\mathrm{AA})$ copolymers, where PAzo is a hydrophobic methacrylate-based azobenzene containing side-chain liquid crystalline polymer, and p(tBA-AA) stands for the weakly hydrophilic poly-(tert-butyl acrylate-co-acrylic acid) polymer. ${ }^{[78]}$ Upon UV-irradiation, the hydrophilicity switch of the PAzo block from hydrophobic to hydrophilic, causes a change in the hydrophilic/hydrophobic balance of the copolymer inducing vesicle dissociation. Although the aggregates formed were coined as vesicles, no characterization data besides SEM pictures are given to support it. The study would require further evidence using techniques such as TEM or light scattering to be more convincing. In addition, the authors did not study encapsulation of model hydrophilic compounds.

Using the same chromophore, Han et al. ${ }^{[71]}$ synthesized a poly( $\mathrm{N}$-isopropylacrylamide)block-poly\{6-[4-(4-pyridyazo)phenoxy] hexylmethacrylate\} (PNIPAM-b-PAzPy) block copolymer, sensitive both to UV and temperature cues. When hydrated, those blocks form giant vesicles with diameters ranging from 1 to $8 \mu \mathrm{m}$. When light was directed to the solution, the authors observed a significant swelling of the vesicles (up to $17 \%$ in volume). This reversible swelling-shrinking behavior was attributed to the isomerization flip of the azobenzene chromophores in the membrane. The cis configuration has a larger surface area when compared with the trans configuration, and this contributes to the deformation of the membrane. According to the authors, the concept of swelling-shrinking is applicable to the transformation energy from light to mechanical energy.

Still using azobenzene derivatives, Lin et al. ${ }^{[72]}$ reported a novel photoresponsive polymersome, obtained by self-assembly of a copolymer composed of hydrophilic poly(ethylene oxide) (PEO) and hydrophobic azopyridine containing poly(methacrylate) (PAP). The vesicles have a mean diameter of 420nm. Upon UV-exposure, several morphology changes were observed, and were described as a cycle including transitions from initial vesicles to larger vesicles via fusion, disintegration and rearrangements. Again, those transitions result from deformation of the membrane structure due to the isomerization of azopyridine moieties disturbing the tight packing of the polymer chains in the membrane. Although the ability of the polymersomes to encapsulate and release hydrophilic drugs was not studied, the authors 
emphasize that the inner content of the vesicles would certainly escape, or would be exchanged with the outer media upon the morphological rearrangements.

Recently, Mabrouk et al. ${ }^{[73]}$ reported on a very original light-responsive system. They fabricated polymeric vesicles in the micrometer-size range, with asymmetric membranes composed of inert poly(ethylene glycol)- $b$-polybutadiene (PEG- $b$-PBD), and a liquid crystalbased copolymer, PEG- $b$-PMAazo444 (PAzo). Upon self assembly, the PEG-PBD copolymers are segregated in the inner leaflet of the membrane, while the PAzo copolymers compose the outer leaflet of the membrane, hence forming an asymmetric membrane. When the Azo moieties are in the trans form, the PAzo polymer adopts a rod-like structure in the membrane. When light is switched on, Azo moieties are in the cis form, and the PAzo polymers undergo a conformational change to reach a coil conformation. Subsequently, the volume occupied by the PAzo chains is increased, leading to a spontaneous change in curvature and to the burst of the giant vesicles by "curling" of the membrane. The photoinduced degradation mechanism indicates that those polymersomes could be used to encapsulate and release a hydrophilic payload.

In light of the short review we proposed above, it seems that there is room for development of novel amphiphilic block copolymers able to self-assemble into light-responsive polymersomes for drug delivery applications. Focusing on light-responsive polymersomes, it is worthy to note that most of the groups cited previously worked on micron size vesicles. As stated before, size is an important feature in the design of drug delivery system, since it is directly linked to its blood clearance and circulation time. To the best of our knowledge, only one report of effective drug release from light-responsive vesicles with diameter below 300nm has been published so far. ${ }^{[79]}$ This work reported by Katz et al. is discussed in detail further in this thesis.

\subsection{Scope of the thesis}

\subsubsection{Objective and motivation}

The main objective of this study is to combine the stimuli-responsiveness of special copolymers with the ability to generate vesicles by self-assembly, in order to obtain a smart, 
light-sensitive nanocarrier. In this respect, we designed a new amphiphilic and light-sensitive block copolymer: $\operatorname{poly}(\gamma$-methyl- $\varepsilon$-caprolactone)-ONB-poly(acrylic acid) (PMCL-ONB-PAA). The self-assembled structures obtained from this diblock should be able to encapsulate an active compound, and release it upon the application of a light trigger, making them ideal candidates for drug delivery applications.

\subsubsection{Approach and concept}

Works on light responsive systems were briefly presented above. The problems encountered by Y.Zhao and coworkers, ${ }^{[64]}$ and B.Zhao and coworkers, ${ }^{[7]}$ are important to our strategy, since we will also use an ONB derivative as the sensitive molecule. Namely, in both studies the authors do not discuss the toxic effect of the released O-nitrosobenzaldehyde, known to be highly reactive toward other molecules such as proteins. ${ }^{[80,81]}$ In order to avoid the toxicity of their systems, we introduced a photolabile group as a linker between the hydrophobic and hydrophilic polymer chains that will drastically reduce the amount of ONB in the system. This approach was investigated by Moon et $a l .^{[82]}$ to produce ONB-poly(styrene)- $b$-poly(ethylene oxide) (ONB-(PS- $b$-PEO)) and by Fustin et $a l .{ }^{[83]}$ to produce PS-hv-PEO and poly(styrene)-hvpoly(methyl methacrylate) (PS-hv-PMMA). However, the synthetic strategy used by these groups is based on coupling chemistry, usually not a well-controlled and tunable way to obtain block copolymers. Here we present a different approach: the synthesis and use of an outstanding difunctional photosensitive initiator allowing the growth of polymer chains from two different functionalities. In addition, this approach gives us the possibility to introduce also $\mathrm{pH}$ responsiveness in our system, with the use of tolerant ATRP and ROP living polymerization techniques.

The structure of the amphiphilic block copolymer is based on the photocleavable moiety (O-nitrobenzyl) acting as a junction point between the hydrophilic poly(acrylic acid) (PAA) and hydrophobic poly $(\gamma$-methyl- $\varepsilon$-caprolactone) (PMCL) blocks (Figure 8). 


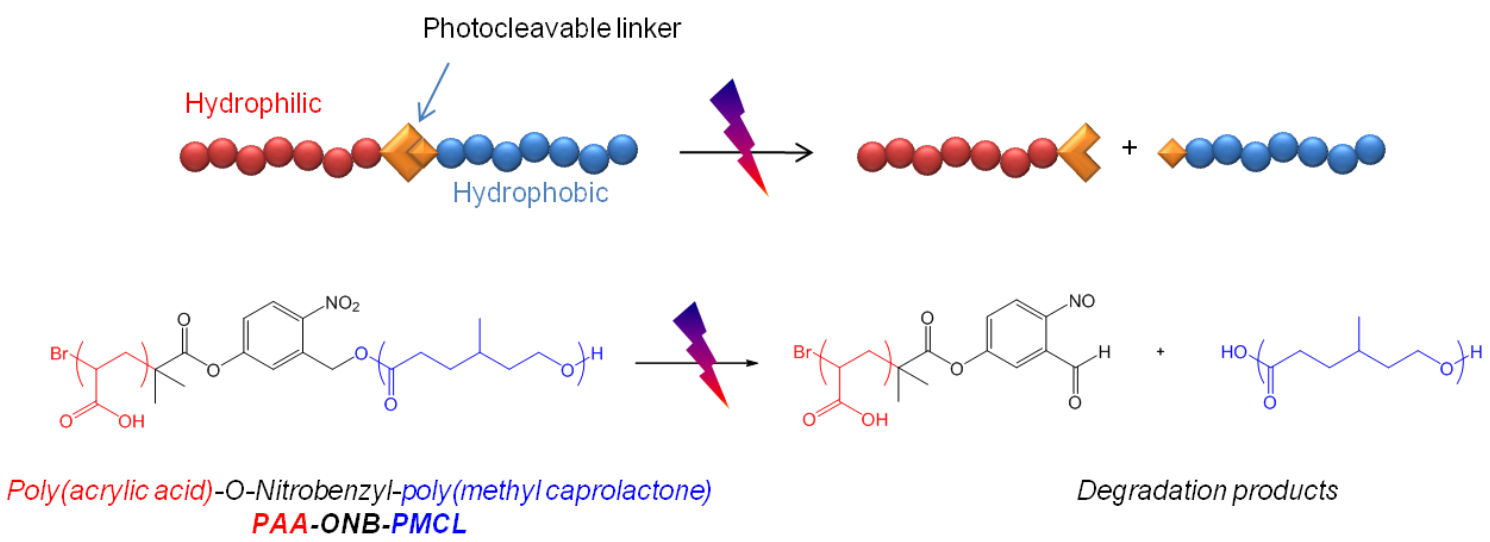

Figure 8: Schematic view of the amphiphilic photocleavable block copolymer, chemical structure of the poly(methyl caprolactone)-ONB-poly(acrylic acid) diblock copolymer, and its degradation products upon UV irradiation. Upon cleavage, PAA chains bearing the photodegraded linker and PMCL chains bearing - $\mathrm{COOH}$ end groups are formed.

The copolymers form well defined supramolecular structures in aqueous media, including vesicles which can be loaded with hydrophilic molecules. The strategy of payload release from our system is based on exposing vesicles to UV light, inducing a subsequent degradation of the ONB linkers that cause the cleavage of corona-forming PAA chains, and exposing the PMCL domains to water (Figure 9). Because they are energetically unfavorable, these photodegraded supramolecular structures undergo a rearrangement at the macromolecular level to yield more favorable entities, or the hydrophobic domains simply precipitate. The inner content of the vesicles is released during this transition (Figure 9). 


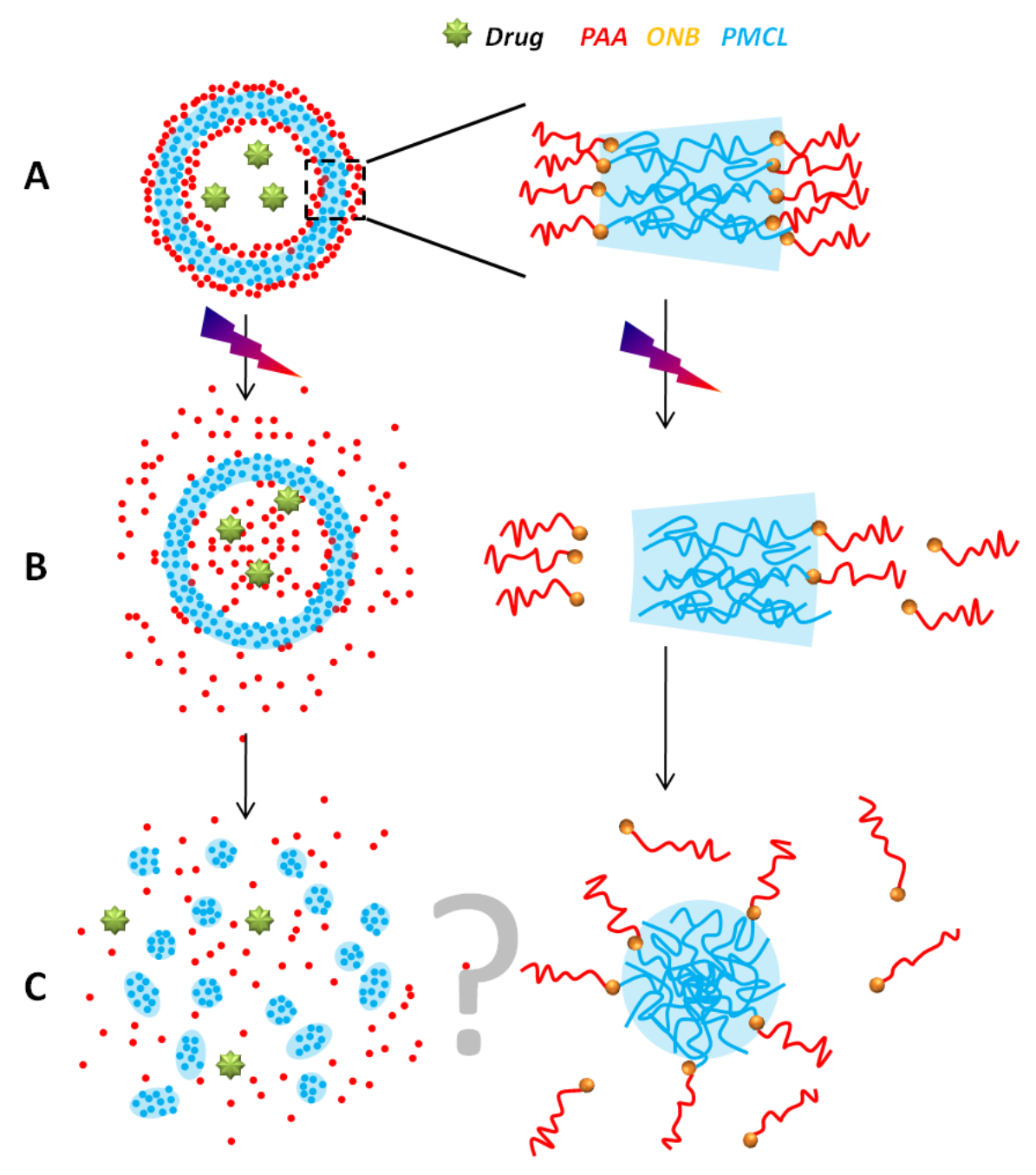

Figure 9: Scheme depicting the polymersomes and the conformation of the assembled polymer chains forming its membrane (A). Upon UV exposure, the corona PAA chains are cleaved, i.e. they are separated from the PMCL forming the core of the membrane $(B)$. Consequently, the vesicle's membrane is destroyed and, presumably, the payload released $(C)$.

The first chapter of this thesis describes the synthesis of a new photocleavable diblock copolymer using living polymerization techniques providing control over molecular weight (Mw) and polydispersity index (PDI).

In the second chapter, we report on the UV light-induced degradation of the polymer chains in solvent and in aqueous media, using simple characterization techniques (ultraviolet- 
visible (UV-Vis) spectrometry, gel permeation chromatography (GPC), and nuclear magnetic resonance (NMR)).

In the third chapter, we investigate the self-assembly behavior of the polymer chains in aqueous solution, and since elucidating the UV degradation mechanism of our vesicles is a key step before investigating their potential as triggerable nanocarriers, we carefully study the change in size and morphology of the self-assembled structures upon UV exposure.

In the last chapter, we demonstrate the ability of the system to encapsulate and release a payload upon UV-trigger, in other words, its ability to work as smart nanocarrier for drug delivery applications. In this respect we investigate the conditions in which the polymer vesicles allow encapsulation and release of a variety of compounds, ranging from small molecules (dyes) up to proteins (eGFP), in mild conditions. 


\section{Synthesis of new photocleavable block copolymers}

Various functional moieties and chemical bonds have been introduced in diblock copolymers as a junction point between the two blocks via diverse synthesis routes.

A commonly used approach involves the use of a macroinitiator, i.e. a starting polymer bearing one or more active sites, which can then initiate the growth of a second block. The active site is usually obtained through the modification of the end-groups of a preexisting homopolymer. A necessary requirement is to not affect the functionality of the linker by the new chemical bond created upon polymerization. Several examples of this approach can be found in literature, including diblocks bearing a photocleavable, ${ }^{[82,84]}$ a redox sensitive, ${ }^{[85-88]}$ or an acidlabile bond. ${ }^{[50,89]}$

A second approach, which can be referred to as "post-polymerization", consists in connecting two preexisting homopolymers. The responsiveness of the linker is usually a result of the reaction attaching the two blocks (coupling or simple chemistry reaction). With this technique, redox sensitive, ${ }^{[90]}$ and light-sensitive, ${ }^{[91]}$ moieties were introduced via direct coupling of homopolymers bearing functional end-groups.

In the third technique, here referred as "the difunctional linker approach", both polymer segments are grown directly from the sensitive linker. The challenging point comes from the requirement to use a molecule which, in addition to its responsive property (to any stimulus), bears two different functionalities to attach polymer segments to both sides. Due to the inherent complexity of such a linker, especially in terms of chemical stability, the technique was seldom used to introduce stimuli-sensitive moieties in block copolymers. ${ }^{[92]}$ Despite the lack of previous studies, we chose to work with this versatile and elegant approach.

\subsection{The difunctional linker approach}

The synthetic approach developed in this work is shown in Figure 10. We first synthesized a difunctional initiator bearing a hydroxyl group and a tertiary bromine group 
(ONB). In the difunctional initiator approach, a first block (A) is grown from the $\mathrm{Y}$ functionality $(\mathrm{OH})$, yielding a homopolymer called macroinitiator. In the second step, the $\mathrm{X}$ functional group from the macroinitiator $(\mathrm{Br})$ reacts with a second monomer, yielding the desired $\mathrm{AB}$ diblock copolymer.

1)

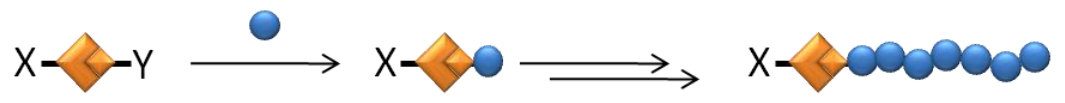

2)

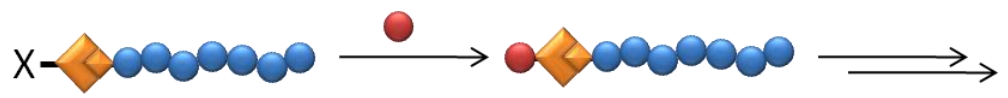

Figure 10: Scheme of AB diblock copolymer synthesis with the difunctional initiator approach.

The use of a dual initiator provides many advantages over classical methods for block copolymer synthesis, such as coupling reaction of the preformed homopolymers. In particular, the presence of two different initiation sites offers the possibility to combine the growth of mechanistically incompatible polymer chains. ${ }^{[93]}$ In order to have a better control over the two concurrent living polymerization techniques, we designed a dual initiator capable of initiating ring opening polymerization (ROP) and atom transfer radical polymerization (ATRP) in a sequential two-step process (Figure 11).

The dual initiator approach using the ROP-ATRP combination is well documented for a number of systems, using either a one-step procedure, ${ }^{[94-96]}$ or a two-step process. ${ }^{[6-99]}$ Amphiphilic block copolymers were synthesized using this synthesis route and, as emphasized by Bernaerts $e t a l$. in a review on heterofunctional initiators, ${ }^{[93]}$ according to a totally controlled procedure. 


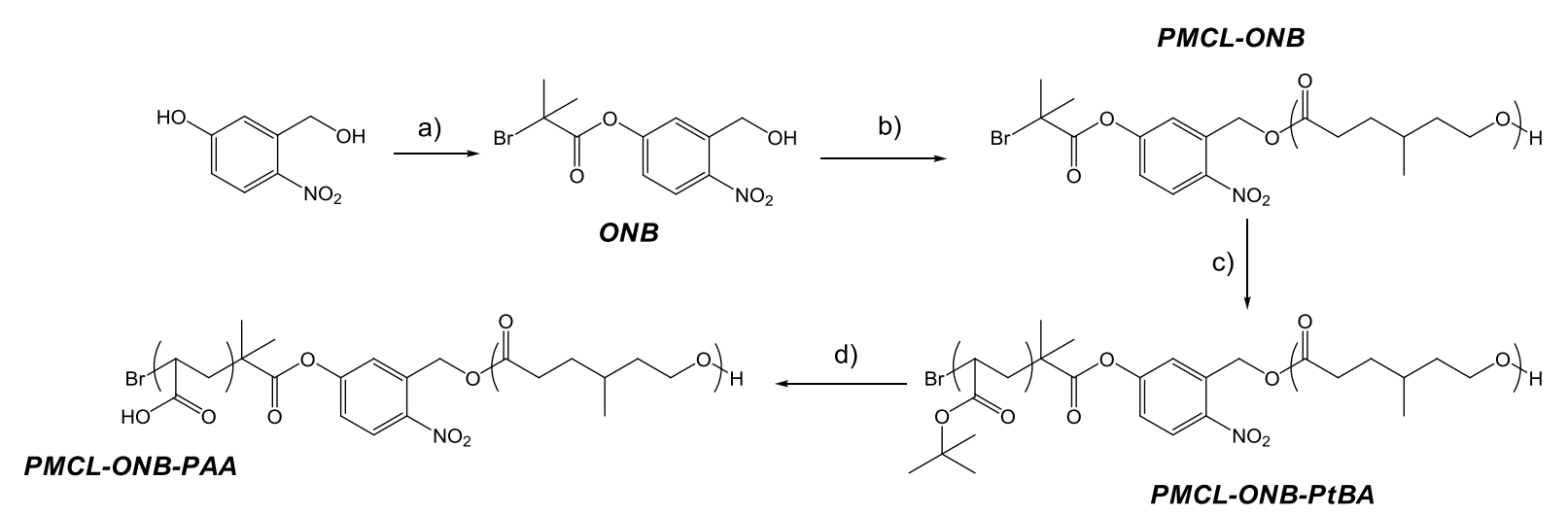

Figure 11: Synthetic procedure for PMCL-ONB-PAA diblock copolymers. a) BiBB (0.67 eq),

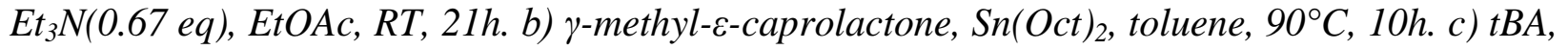
$\mathrm{CuBr} / \mathrm{PMDETA}$, toluene, $75^{\circ} \mathrm{C}$. d) TFA (15 eq/tBA unit), $\mathrm{CH}_{2} \mathrm{Cl}_{2}, \mathrm{RT}$, $18 \mathrm{~h}$.

The ONB hydroxyl group served as initiator for the ring opening polymerization of $\gamma$ -

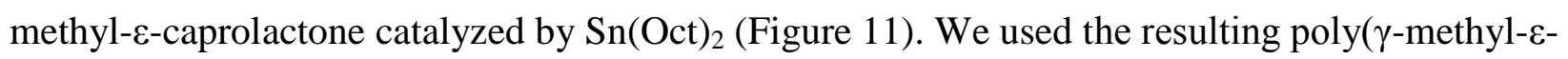
caprolactone) (PMCL-ONB) macroinitiator (bearing a tertiary bromine end group) to polymerize tert-Butylacrylate (tBA) by atom transfer radical polymerization with $\mathrm{CuBr}$ as catalyst and PMDETA as ligand, yielding the diblock PMCL-ONB-PtBA. The final diblock PMCL-ONBPAA was obtained upon deprotection of the tBA units with trifluoroacetic acid.

\subsection{Synthesis of a new photocleavable moiety}

We chose to work with an O-nitrobenzyl linker as the photoresponsive molecule, due to its chemical stability and rapid cleavage by near-UV irradiation (wavelength $>320 \mathrm{~nm}$ ). Another interesting feature of O-nitrobenzyl is its ability to be cleaved by two-photon near infrared (NIR, $700-1000 \mathrm{~nm}$ ) irradiation. NIR light is particularly attractive for biomedical applications compared to UV light; at these longer wavelengths, the irradiation is less harmful to cells, and the penetration depth into tissues is significantly increased. ${ }^{[100]}$ Additionally, ONB has been widely used and studied as a photolabile protective group. ${ }^{[101-104]}$ 
The photocleavable linker was prepared according to a method proposed elsewhere, ${ }^{[98,}$ 105] and adapted to the light-sensitive O-nitrobenzyl diol. A commercially available diol, 5hydroxy-2-nitrobenzyl alcohol, was reacted with $\alpha$-bromoisobutyryl bromide (Figure 11). The esterification occurred preferentially at the phenol position yielding the desired compound. However, it should be noted that a mixture of compounds was obtained due to the formation of diester byproduct. To minimize the formation of this compound, we used a 1.5 fold excess of the diol. The remaining impurities and unreacted diols were removed by flash chromatography. We characterized the initiator and by-products by ${ }^{1} \mathrm{H}$ NMR and ${ }^{13} \mathrm{C}$ NMR (Figure 12). 

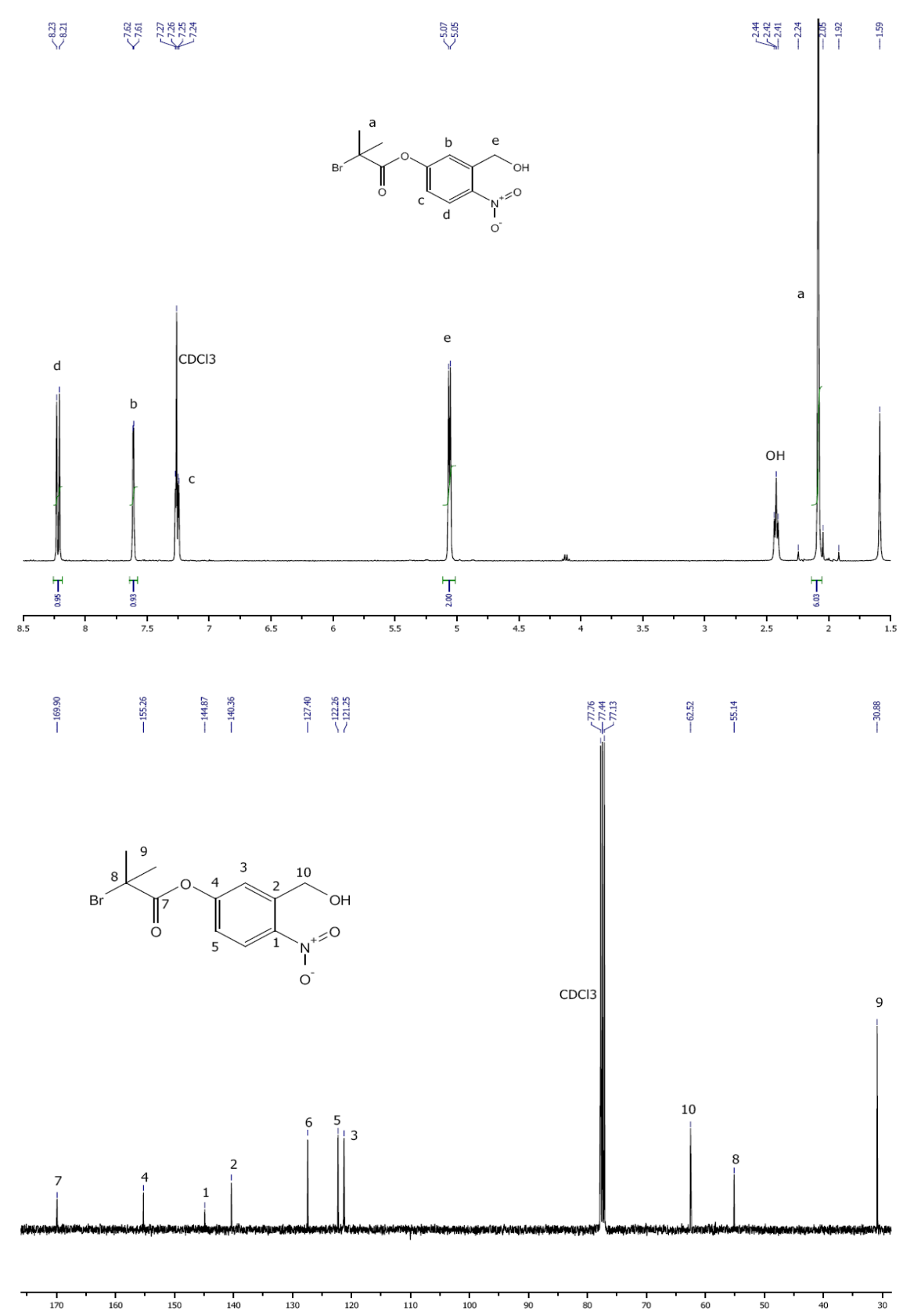

Figure 12: ${ }^{1} \mathrm{H} N M R\left(\right.$ up) and ${ }^{13} C N M R$ spectra of ONB linker. Samples were dissolved in $\mathrm{CDCl}_{3}$.

In ${ }^{1} \mathrm{H}$ NMR, we see the three peaks corresponding to the three aromatic protons (c: $\delta=$ 7.25ppm, b: $\delta=7.61 \mathrm{ppm}$, and $\mathrm{d}: \delta=8.22 \mathrm{ppm}$ ), the two protons of the $-\mathrm{CH}_{2}$ - linked to the $\mathrm{OH}$ 
(e: $\delta=5.06 \mathrm{ppm}$ ), and the six protons of the methyl groups (a: $\delta=2.08 \mathrm{ppm}$ ). The sharp signal of the proton of the alcohol group $(\delta=2.42 \mathrm{ppm})$ is due to proton exchange with water molecules $(\delta$ $=1.59 \mathrm{ppm}) . \mathrm{In}{ }^{13} \mathrm{C} \mathrm{NMR}$, all peaks were assigned to carbon atoms as follows; $\delta_{\mathrm{C}}(\mathrm{ppm}): 30.8$

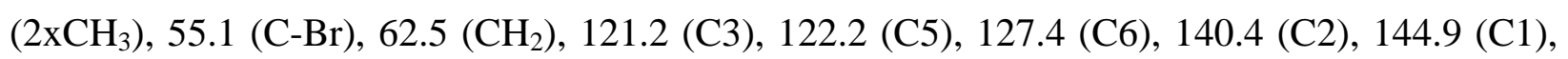
$155.3(\mathrm{C} 4), 169.9(\mathrm{C}=\mathrm{O})$. Both spectra reveal the expected structure, with no traces of byproducts.

\subsection{PMCL-ONB-PAA diblock synthesis}

We will detail the ROP-ATRP combination we used to synthesize PMCL-ONB-PAA diblock copolymer.

\subsubsection{ROP of $\varepsilon$-caprolactone and $\gamma$-methyl- $\varepsilon$-caprolactone monomers initiated by ONB}

\subsubsection{Introduction}

Cyclic esters are known to polymerize via anionic or cationic polymerization to form polyesters. In the case of anionic polymerization, initiators such as metal alkoxides $\left(\mathrm{Al}(\mathrm{OR})_{3}\right.$ for instance) and metal carboxylates (tin(II) 2-ethylhexanoate, $\left.\mathrm{Sn}(\mathrm{Oct})_{2}\right)$ are used. ${ }^{[106]}$ Although aluminum based alkoxides are among the most used initiators, we could not prepare them with our ONB alcohol. Indeed, aluminum is known to react with nitro compounds, ${ }^{[107]}$ and would potentially destroy the photosensitivity of our linker by alteration of the nitro functional group. Therefore we used tin(II) 2-ethylhexanoate as the catalyst. In ROP with $\mathrm{Sn}(\mathrm{Oct})_{2}$, the actual initiator is the metal alkoxide formed by the reaction between metal carboxylate and alcohol (Figure 13, A). 


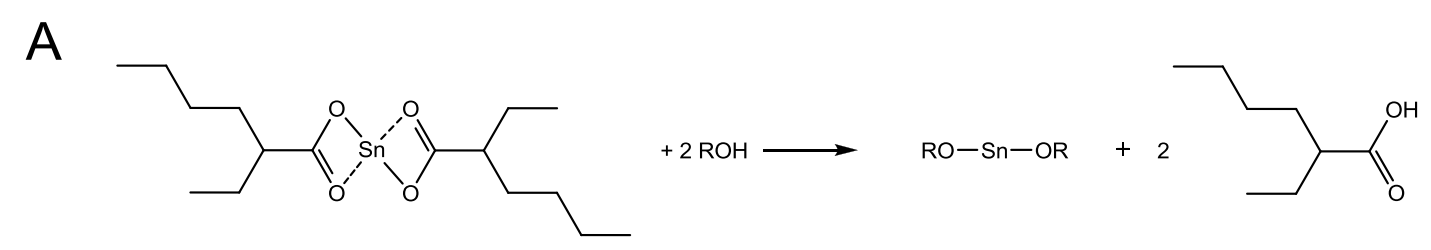

B
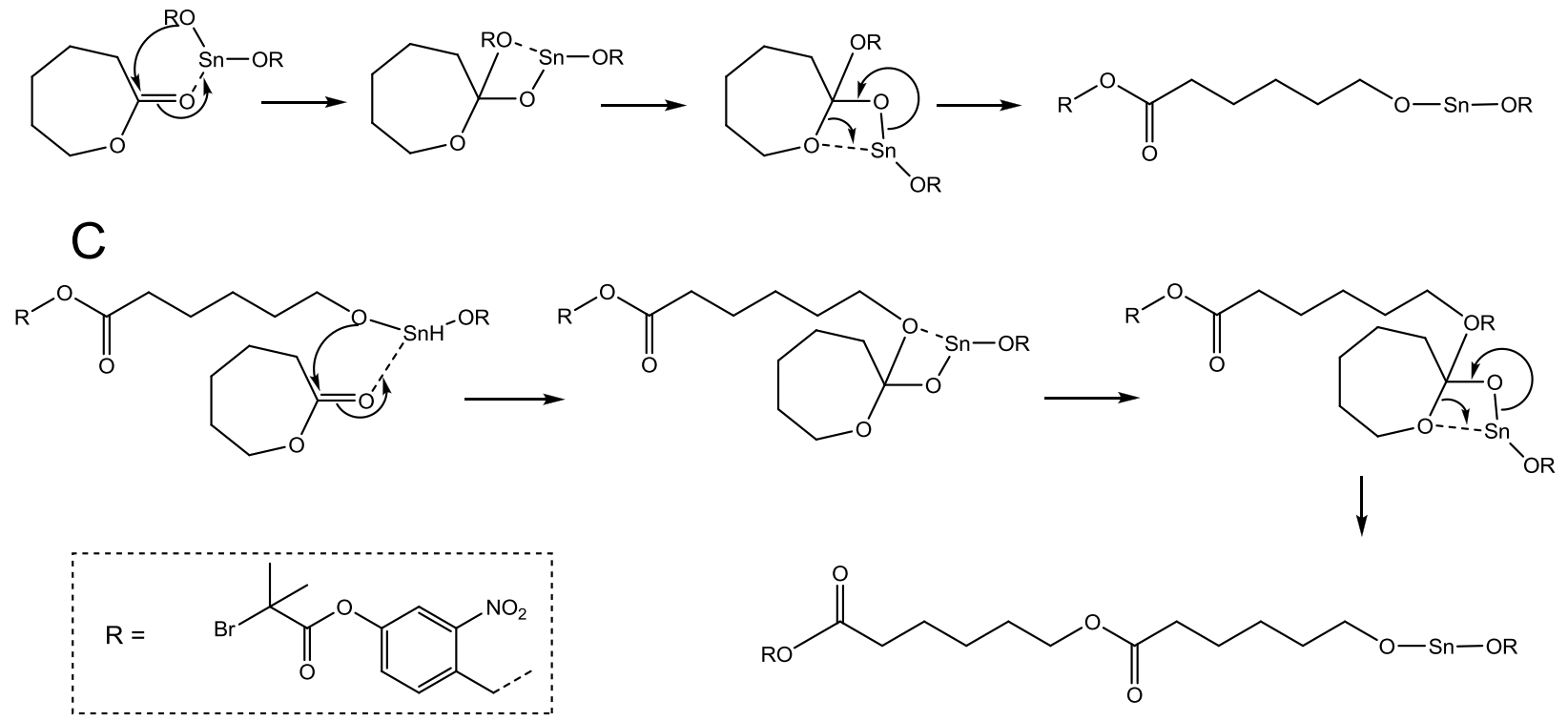

Figure 13: Mechanism of initiation in stannous octoate catalyzed polymerization of $\varepsilon$ caprolactone, including (A) formation of stannous alkoxide initiator, (B) coordination/insertion of monomer into the stannous alkoxide bond, and $(C)$ chain extension. ${ }^{[108]}$

The polymerization of cyclic esters initiated with $\mathrm{Sn}(\mathrm{Oct})_{2}$, proceeds by a coordinationinsertion mechanism, with propagation (Figure 13), and has been described as a living polymerization. ${ }^{[108-110]}$

The first copolymerization experiments were performed with $\varepsilon$-caprolactone as monomer, yielding poly(caprolactone) (PCL) hydrophobic blocks. We took advantage of this commercially available monomer to verify the feasibility of the ROP-ATRP procedure with the new ONB linker. However, poly(caprolactone) is a crystalline polymer with reduced chain mobility, and its use as the hydrophobic segment in self-assembling amphiphilic block copolymers does not confer flexibility to the formed superstructures. Foreseeing the potential limitations of such a system in our future applications, and following successful polymerizations, we rapidly switched 
to a similar monomer, $\gamma$-methyl- $\varepsilon$-caprolactone. This monomer is synthesized by Bayer-Villiger oxidation, ${ }^{[41]}$ and can be polymerized (with ROP) under the same conditions to yield poly $(\gamma$ methyl- $\varepsilon$-caprolactone). In the case of PMCL, a totally amorphous homopolymer, ${ }^{[11]}$ the flexibility of the chains is such that it provides fluidity to the polymer membrane formed upon self-assembly, a desirable property for polymersomes. Unless specified otherwise, the results and discussions reported in this thesis were obtained with, and refer to, PMCL-ONB-PAA block copolymers.

\subsubsection{Results}

The PMCL-ONB macroinitiators were characterized by ${ }^{1} \mathrm{H}$ NMR and GPC. The ${ }^{1} \mathrm{H}$ NMR spectrum of the PMCL-ONB polymer shows the complete shift of the methylene protons of ONB from $\delta=5.06 \mathrm{ppm}$ (see Figure 12, labeled e) to $\delta=5.53 \mathrm{ppm}$ (Figure 14, labeled e), indicating that the $\mathrm{OH}$ group reacted and that the polymerization process was effectively initiated by the difunctional linker. The PMCL backbone protons have chemical shifts from 0.92 up to $4.11 \mathrm{ppm}$, different from the cyclic lactone monomer. The $\mathrm{k}$ protons $(\delta=4.11 \mathrm{ppm})$, are shifted toward high fields (high chemical shift) because they are on the $\mathrm{CH}_{2}$ linked to an oxygen atom. f protons $(\delta=$ $2.31 \mathrm{ppm}$ ) are also shifted toward high fields due to the vicinity of the electron-rich ester linkage. The protons of the methyl group of the backbone are unshielded (i, lower fields, $\delta=0.92 \mathrm{ppm}$ ). The other backbone protons (g, h, and $\mathrm{j}$ ) have similar chemical shifts, from 1.45 to $1.68 \mathrm{ppm}$. The spectrum also shows the peaks associated to the initiator described above. 

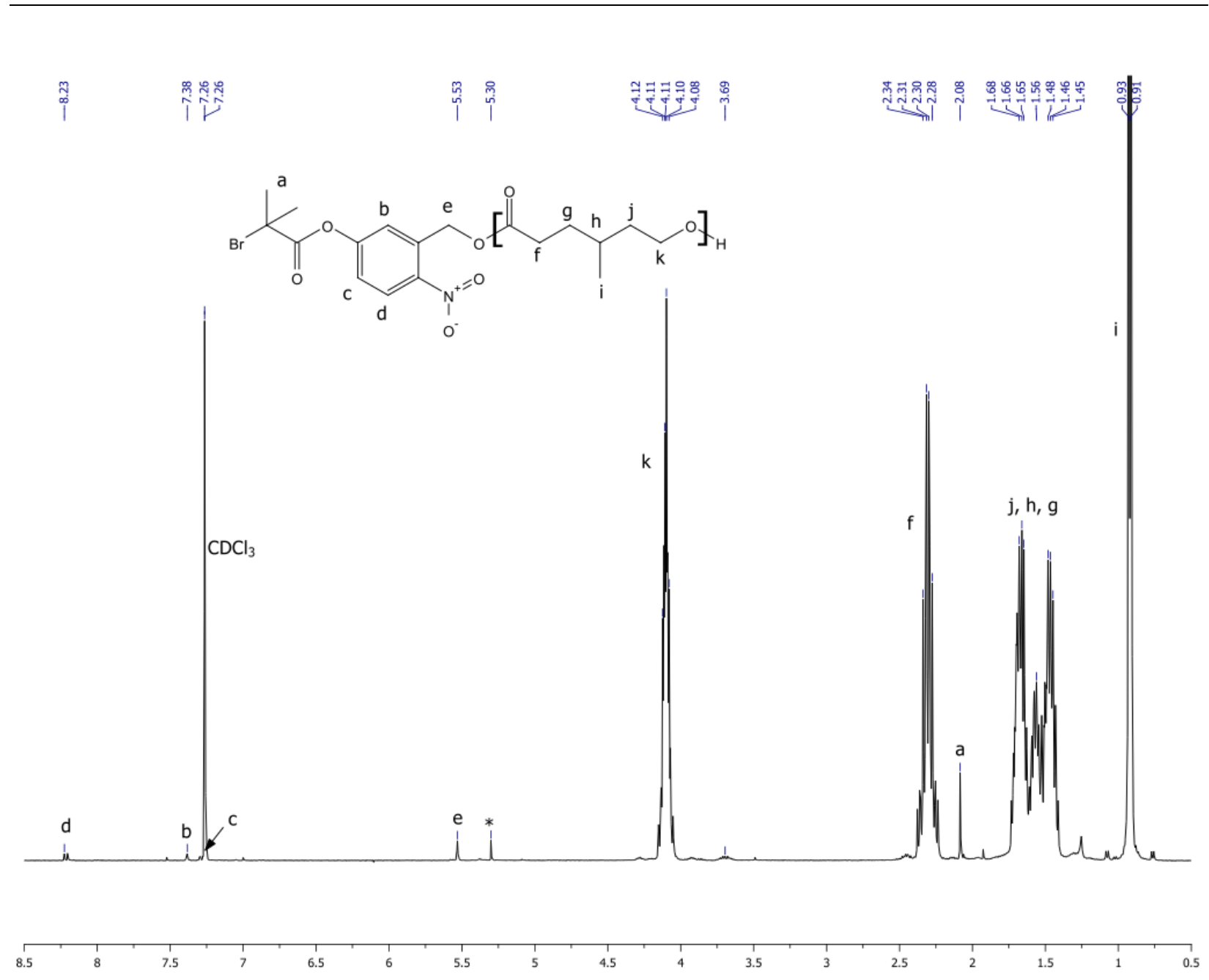

Figure 14: Typical ${ }^{1} \mathrm{H}-\mathrm{NMR}$ spectrum for PMCL-ONB macroinitiator dissolved in $\mathrm{CDCl}_{3}$.

We estimated the degree of polymerization by comparing the integrals of the methylene protons peak (e) (or the 6 protons from the methyl signal, labeled a) of the initiator, with the methylene protons of the monomer $(\mathrm{k})$. These estimations are in good agreement with the theoretical values calculated from the monomer to alcohol ratio which controls the degree of polymerization (see Table 2). ${ }^{[108,112]}$

In addition, the polydispersity indexes obtained by GPC range from 1.1 to 1.3 , thereby confirming that the polymerization was well controlled. Typical chromatograms are shown in Figure 15. 

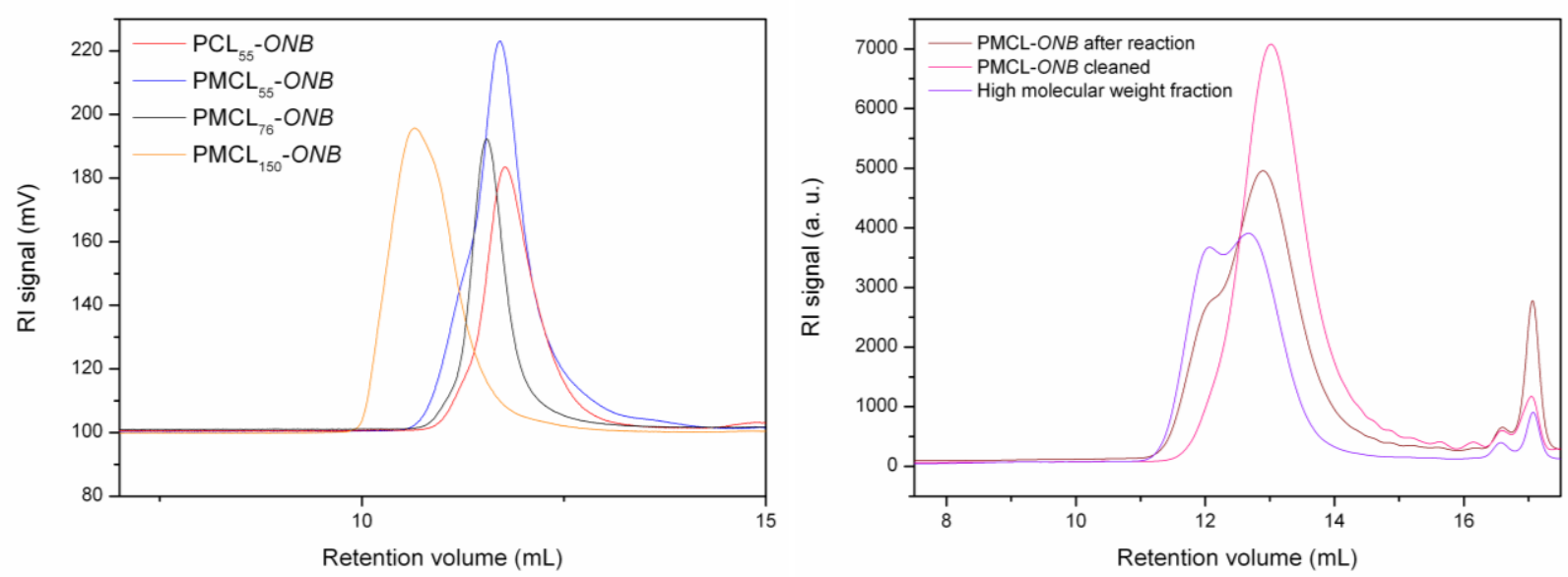

Figure 15: (Left) typical GPC chromatograms for PMCL-ONB and PCL-ONB macroinitiators. For PMCL ${ }_{55^{-}} \mathrm{ONB}$, the shoulder corresponds to high molecular weight polymer chains initiated by water; (Right) GPC traces for a PMCL-ONB homopolymer, showing the successful separation of high MW water initiated chains from the ONB initiated chains.

For a couple of polymer synthesis, the presence of water in non-negligible quantities, contributed to the competitive initiation of polymer chains. In this case, the reaction cannot be considered as a living polymerization. The polymer chains initiated by water have higher molecular weights, resulting in peak broadening and an increase in PDI. However, in most cases, it was possible to avoid high molecular weight chains through repeated precipitation steps (Figure 15). It should be noted that water initiated chains lack the ONB linker, and therefore are unable to initiate ATRP in the next step. PMCL-ONB and PCL-ONB blocks synthesized are summarized in Table 2. 
Table 2: Molecular characteristics of synthesized PCL-ONB and PMCL-ONB macroinitiators.

\begin{tabular}{|c|c|c|c|c|c|c|}
\hline Polymer & $\mathrm{N}_{\mathrm{th}}{ }^{\mathrm{a}}$ & $\mathrm{N}(\mathrm{NMR})^{\mathrm{b}}$ & $\mathrm{M}_{\mathrm{n}}(\mathrm{NMR})^{\mathrm{c}}$ & $\mathrm{M}_{\mathrm{n}}(\mathrm{GPC})^{\mathrm{d}}$ & $\mathrm{M}_{\mathrm{w}}(\mathrm{GPC})^{\mathrm{e}}$ & $\mathrm{PDI}^{\mathrm{f}}$ \\
\hline $\mathrm{PCL}_{55}-\mathrm{ONB}$ & 50 & 55 & 6600 & 12600 & 15900 & 1.26 \\
\hline $\mathrm{PMCL}_{55}-\mathrm{ONB}$ & 60 & 55 & 7350 & 10100 & 16200 & 1.6 \\
\hline $\mathrm{PMCL}_{23}-O N B$ & 20 & 23 & 3200 & 2900 & 3300 & 1.15 \\
\hline $\mathrm{PMCL}_{30}-O N B$ & 30 & 30 & 4200 & 3200 & 4100 & 1.26 \\
\hline $\mathrm{PMCL}_{105^{-}} \mathrm{ONB}$ & 100 & 105 & 13800 & 16700 & 22000 & 1.31 \\
\hline $\mathrm{PMCL}_{150^{-}} O N B$ & 200 & 150 & 19500 & 22500 & 33800 & 1.5 \\
\hline $\mathrm{PMCL}_{76}-\mathrm{ONB}$ & 100 & 76 & 10000 & 9100 & 10500 & 1.15 \\
\hline
\end{tabular}

${ }^{a}$ Targeted numbers of MCL (or CL) units. ${ }^{\mathrm{b}}$ Number of monomer repeat units in the PMCL (or PCL) block as determined by ${ }^{1} \mathrm{H}$-NMR spectroscopy. ${ }^{\mathrm{c}}$ Number-average molecular weight of polymers as determined by ${ }^{1} \mathrm{H}-\mathrm{NMR}$ spectroscopy. ${ }^{\mathrm{d}, \mathrm{e}}$ Number and weight average molecular weight of polymers as determined by GPC with polystyrene standards ${ }^{1} .{ }^{\mathrm{f}}$ Polydispersity index determined from GPC results.

\subsubsection{ATRP of tBA with PMCL-ONB macroinitiator}

\subsubsection{Introduction}

In the next step, the second block was grown using atom transfer radical polymerization. ATRP was used since it is a robust technique toward functionalities and a versatile living polymerization affording well defined copolymers. ${ }^{[113,114]}$

In ATRP, the radical $(\mathrm{R} \bullet)$ is generated by the abstraction of the halogen atom from an organic halide $(\mathrm{RX})$, catalyzed by a transition metal compound such as cuprous halide $(\mathrm{CuBr})$. The formed reactive radical initiates polymerization. The advantage of ATRP over other radical polymerization arises from the rapid and reversible deactivation of propagating species by the

\footnotetext{
${ }^{1}$ The molecular weights determined by GPC are not absolute molecular weights. They are calculated from a calibration curve obtained with polystyrene standards, and therefore they differ from values obtained by ${ }^{1} \mathrm{H}-\mathrm{NMR}$ spectroscopy.
} 
persistent transition metal complex $\mathrm{CuBr}_{2}(\mathrm{~L})$, maintaining low radical concentration and minimizing normal termination reactions. At all time during the reaction (i.e. monomer $\mathrm{M}$ conversion), active radicals $\left(\mathrm{RM}_{\mathrm{n}} \bullet\right)$ are converted to so-called dormant species $(\mathrm{RM}-\mathrm{Br})$, ensuring control over growth rate and subsequently over molecular weight distribution.

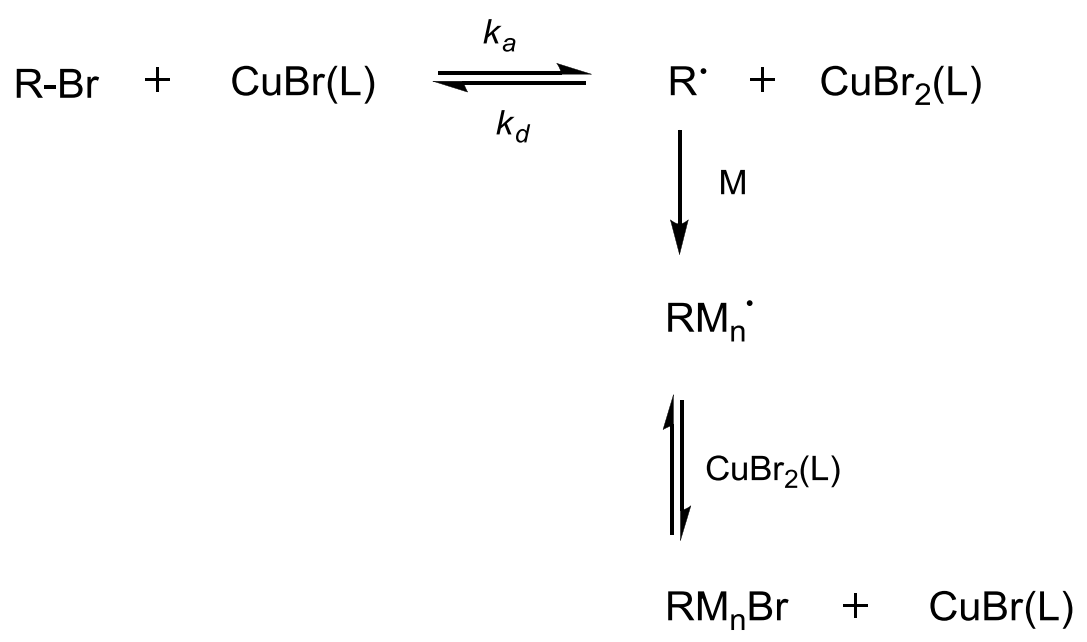

Figure 16: ATRP mechanism catalyzed by cuprous bromide. ${ }^{[106]}$

Although $\mathrm{CuBr}$ is the most frequently used metal compound (Figure 16), a variety of transition metals (copper, iron, ruthenium, nickel) and halides (bromide, chloride, or idodide) can be applied to ATRP.

ATRP is a multi-component system of initiator (RX), activator, deactivator, ligand, and solvent. Therefore the reaction can be affected by a number of parameters, including transition metal, ligand, solvent, and temperature. Depending on the monomer and catalyst, it is necessary to adjust the system and reaction conditions to attain the polymerization conditions mentioned above (i.e. low radical concentration and suppression of normal termination).

Here, the ATRP was conducted with $\mathrm{ONB}$ as the $\mathrm{RX}, \mathrm{CuBr}$ as metal halide, and with $\mathrm{N}, \mathrm{N}, \mathrm{N}$ ',N',N', 'pentamethyldiethylenetriamine (PMDETA) as ligand. Although other ligands were tested (HMTETA and bPy), PMDETA was found to favor solubilization of copper in toluene solution. 


\subsubsection{Results}

The completion of the polymerization is evidenced via ${ }^{1} \mathrm{H}$ NMR with the appearance of the characteristic signals belonging to the acrylate backbone protons ( $\mathrm{m}$ superimposed with $\mathrm{f}, \delta=$ $2.28 \mathrm{ppm}$, and $1, \delta \approx 1.8 \mathrm{ppm})$ and the appearance of the methyl protons from the $\mathrm{tBA}(\mathrm{n}, \delta=$ $1.47 \mathrm{ppm}$, Figure 17).

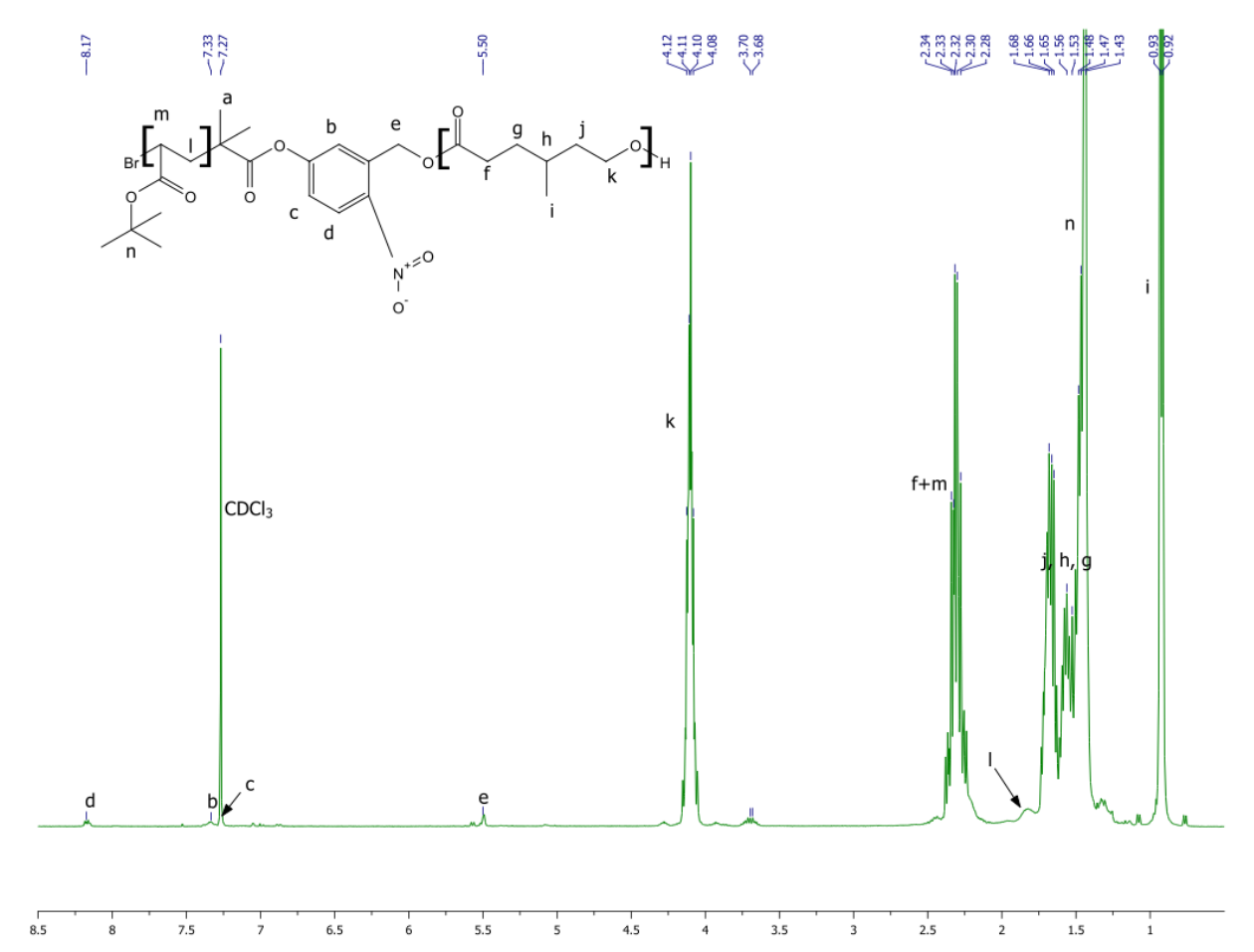

Figure 17: Typical ${ }^{1} \mathrm{H}-\mathrm{NMR}$ spectrum of a PMCL-ONB-PtBA block copolymer dissolved in $\mathrm{CDCl}_{3}$.

The degree of polymerization was calculated from the integral of the PMCL backbone protons $\mathrm{k}$, and the integral of the PtBA backbone proton $\mathrm{m}$ (peak assigned to $\mathrm{f}+\mathrm{m}$ protons).

Although no kinetic study was performed on the ATRP of tBA in the presence of the PMCL-ONB macroinitiator, we can see from the GPC data (Figure 18) that the polymerization 
was well controlled; the diblock copolymer traces are unimodal and symmetrical, no tailing in the low molecular weight region is observed, and the molecular weight distributions are quite narrow (Table 3). The degree of polymerization of the acrylate block was tuned by varying the reaction time. The GPC chromatograms of the copolymer reveal a clear shift toward higher MW when compared to the PMCL-ONB macroinitiator trace.

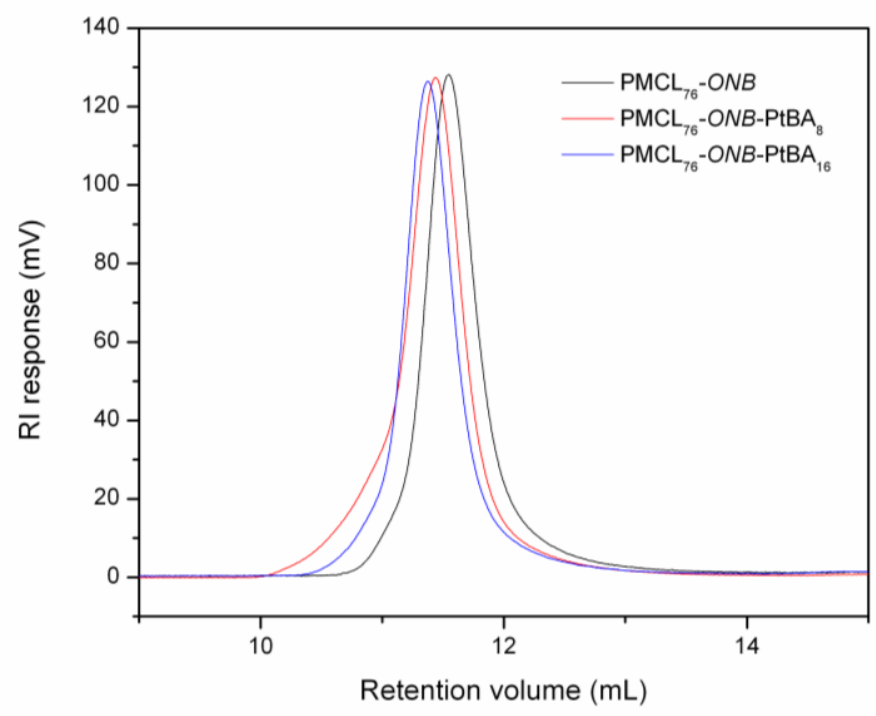

Figure 18: Typical GPC chromatograms showing a PMCL-ONB macroinitiator and two PMCLONB-PtBA diblock copolymers with different molecular weights generated from it.

Table 3: Molecular characteristics of synthesized PMCL-ONB-PtBA copolymers.

\begin{tabular}{lccccc}
\hline Polymer & $\mathrm{N}_{\mathrm{tBA}}(\mathrm{NMR})^{\mathrm{a}}$ & $\mathrm{M}_{\mathrm{n}}(\mathrm{NMR})^{\mathrm{b}}$ & $\mathrm{M}_{\mathrm{n}}(\mathrm{GPC})^{\mathrm{c}}$ & $\mathrm{M}_{\mathrm{w}}(\mathrm{GPC})^{\mathrm{d}}$ & $\mathrm{PDI}^{\mathrm{e}}$ \\
\hline $\mathrm{PCL}_{55}$-ONB-PtBA & 8 & 7600 & 14900 & 20400 & 1.36 \\
$\mathrm{PCL}_{55}$-ONB-PtBA & & 10400 & 19300 & 30800 & 1.59 \\
$\mathrm{PCL}_{55}$-ONB-PtBA & 30 & 7900 & 10300 & 13100 & 1.27
\end{tabular}




\begin{tabular}{|c|c|c|c|c|c|}
\hline $\mathrm{PMCL}_{55^{-}} \mathrm{ONB}-\mathrm{PtBA}_{10}$ & 10 & 8600 & 16000 & 23000 & 1.44 \\
\hline $\mathrm{PMCL}_{23}-\mathrm{ONB}-\mathrm{PtBA} \mathrm{A}_{20}$ & 20 & 5800 & 3900 & 5000 & 1.27 \\
\hline $\mathrm{PMCL}_{30^{-}} \mathrm{ONB}-\mathrm{PtBA}_{6}$ & 6 & 4900 & 3650 & 5100 & 1.39 \\
\hline $\mathrm{PMCL}_{30^{-}} O N B-\mathrm{PtBA}_{10}$ & 10 & 5400 & 3900 & 5500 & 1.39 \\
\hline $\mathrm{PMCL}_{105}-O N B-\mathrm{PtBA}_{30}$ & 30 & 17600 & 23000 & 28000 & 1.21 \\
\hline $\mathrm{PMCL}_{150}-O N B-\mathrm{PtBA}_{10}$ & 10 & 20800 & 22500 & 33200 & 1.48 \\
\hline $\mathrm{PMCL}_{150^{-}} O N B-\mathrm{PtBA}_{23}$ & 23 & 22500 & 24000 & 34900 & 1.45 \\
\hline $\mathrm{PMCL}_{76^{-}} \mathrm{ONB}-\mathrm{PtBA}_{41}$ & 41 & 15290 & 13400 & 19100 & 1.42 \\
\hline $\mathrm{PMCL}_{76^{-}} \mathrm{ONB}-\mathrm{PtBA} \mathrm{A}_{16}$ & 16 & 12350 & 11400 & 14800 & 1.29 \\
\hline $\mathrm{PMCL}_{76^{-}} \mathrm{ONB}-\mathrm{PtBA}_{8}$ & 8 & 11050 & 11300 & 13300 & 1.28 \\
\hline
\end{tabular}

${ }^{a}$ Number of monomer repeat units in the PtBA block as determined by ${ }^{1} \mathrm{H}-\mathrm{NMR}$ spectroscopy. ${ }^{\mathrm{b}}$ Number-average molecular weight of block copolymers as determined by ${ }^{1} \mathrm{H}-\mathrm{NMR}$ spectroscopy. ${ }^{\mathrm{c}, \mathrm{d}}$ Number and weight average molecular weight of block copolymers as determined by GPC with polystyrene standards. ${ }^{\mathrm{e}}$ Polydispersity index determined from GPC results.

\subsubsection{Deprotection of the PtBA block}

To obtain PMCL-ONB-PAA block copolymers, the tert-butyl groups of tBA units are cleaved according to an established method, based on mild acidolysis of the ester bond with an excess of trifluoroacetic acid (TFA, 15 eq./tBA unit). ${ }^{[15,116]}$ The success of the reaction was evidenced by ${ }^{1} \mathrm{H}$ NMR spectroscopy, which shows the disappearance of the tert-butyl signal (n, Figure 17) and the presence of the signal for the acid protons from the deprotected groups at $\delta=$ $10.83 \mathrm{ppm}$ (Figure 19). The polyacrylate backbone protons are also shifted upon deprotection (m from $\delta=2.28 \mathrm{ppm}$ to $\delta=2.46 \mathrm{ppm}$, and 1 from $\delta=1.8 \mathrm{ppm}$ to $\delta=1.96 \mathrm{ppm}$ ). 

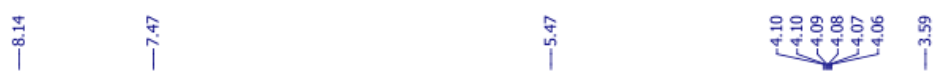

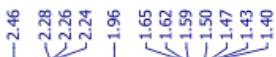
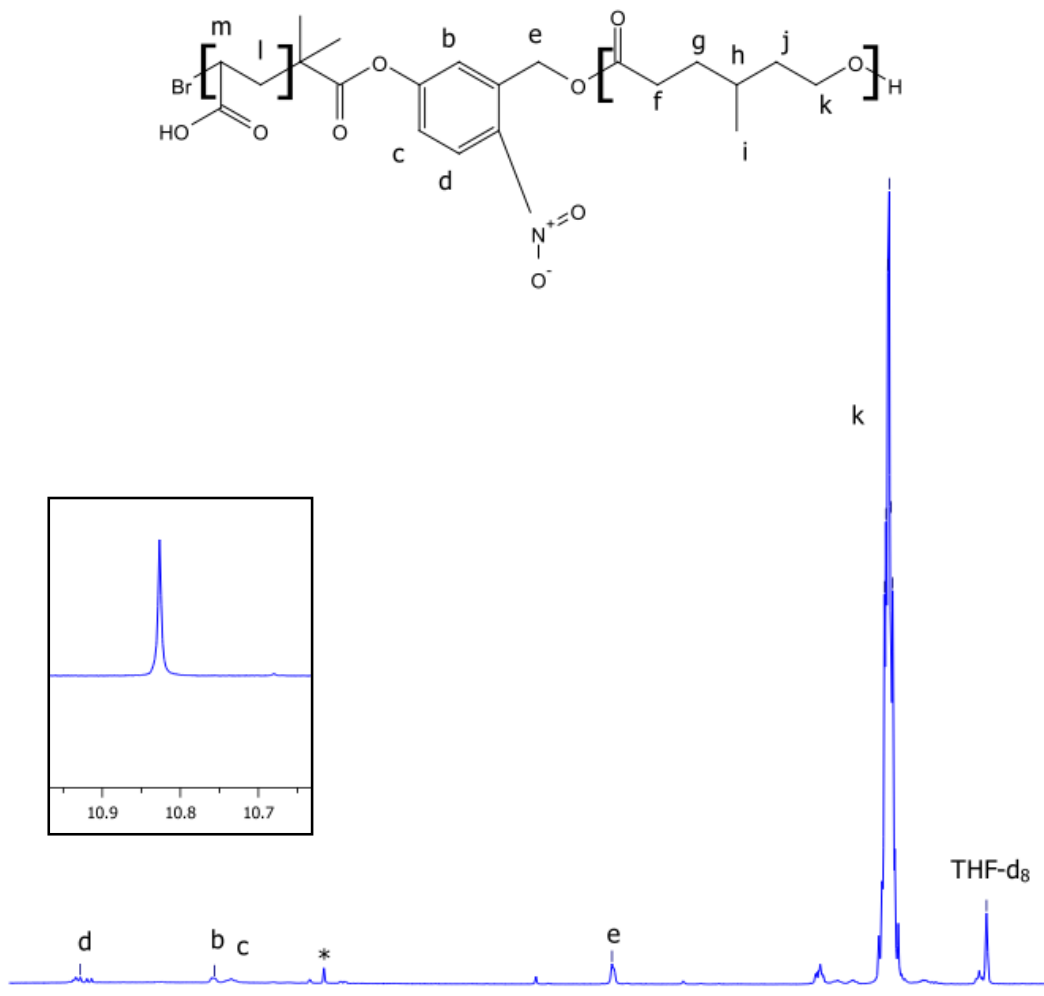

i
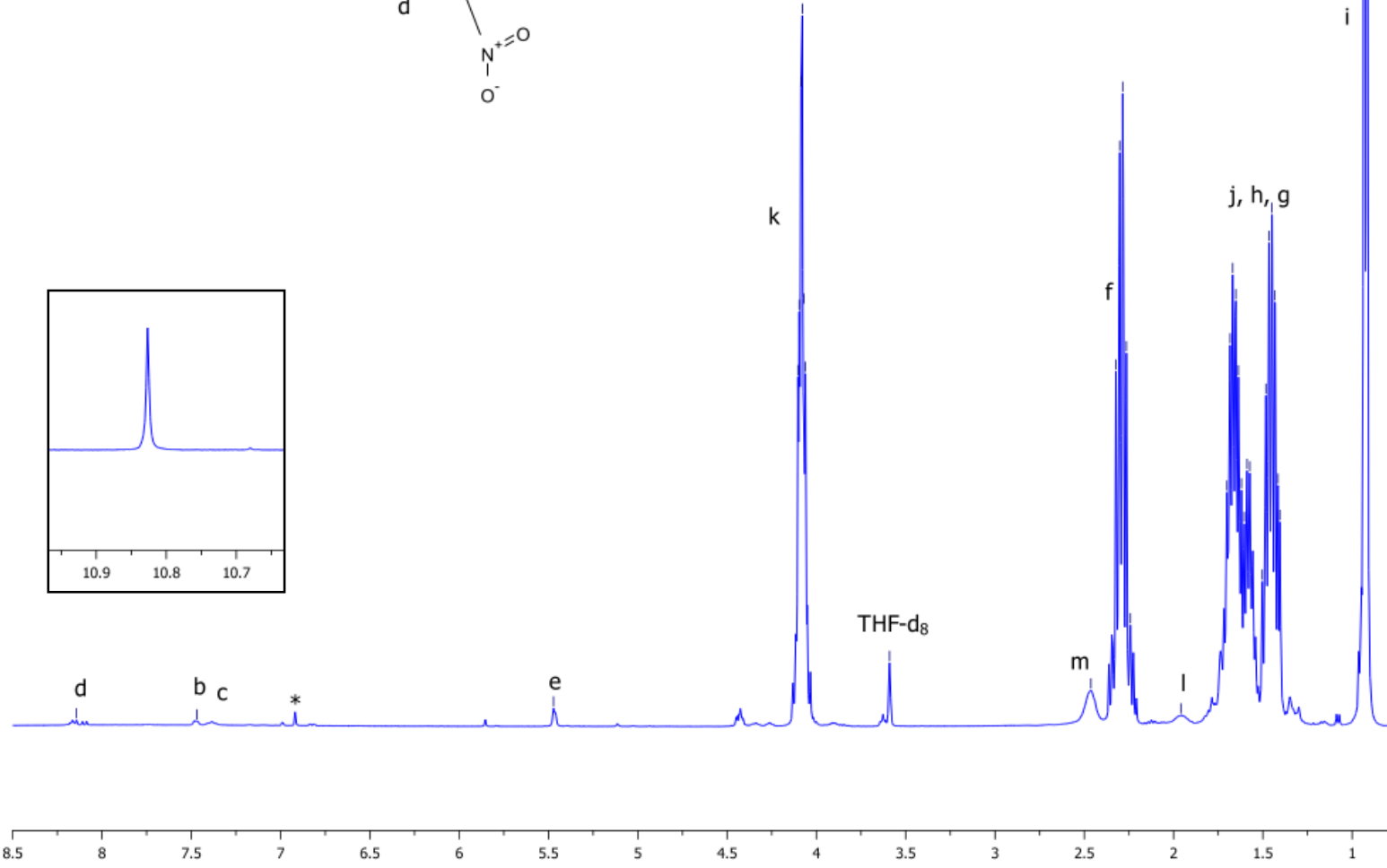

Figure 19: Typical ${ }^{1} H$-NMR spectrum of a PMCL-ONB-PAA block copolymer in deuterated THF. The insert shows the peak associated to the acid protons of the PAA block.

The deprotection was also followed by Infrared spectroscopy. The appearance of a broad peak ranging from 2400 to $3600 \mathrm{~cm}^{-1}$ in the FT-IR spectrum attributed to the $\mathrm{O}-\mathrm{H}$ stretching (Figure 20) confirmed the presence of carboxylic groups as well. Additionally, the $\mathrm{C}=\mathrm{O}$ stretching absorbance band of PMCL-ONB-PAA is shifted to lower wavenumbers (ca. $1700 \mathrm{~cm}^{-}$ $\left.{ }^{1}\right)$ compared to that of PMCL-ONB-PtBA $\left(1730 \mathrm{~cm}^{-1}\right)$. This also results in a broadening of the peak since both signals from PMCL ester carbonyls and PAA acid carbonyls are superimposed. 


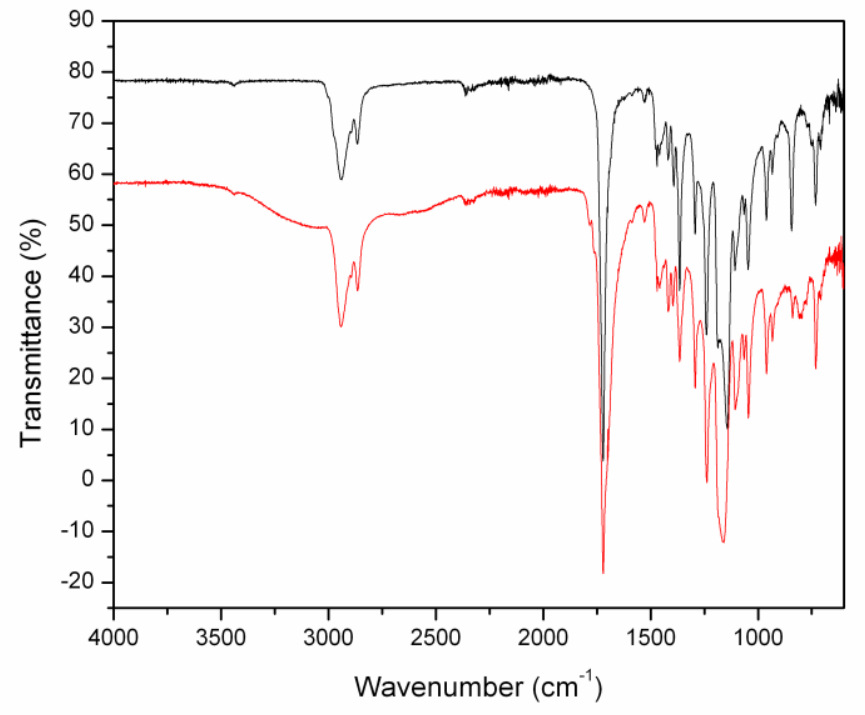

Figure 20: IR spectra of PMCL-ONB-PtBA (black) and its deprotected counterpart, PMCLONB-PAA (red).

Finally, the solubility of the diblock changed during the course of the reaction, thus indicating a change in polarity due to effective deprotection of the PtBA block. It has been reported earlier that under the described deprotection method, no degradation of the polyester block occurred, ${ }^{[117]}$ which is confirmed by ${ }^{1} \mathrm{H}$ NMR. 


\section{Photodegradation of polymer chains}

\subsection{UV-irradiation setup}

The UV irradiation was operated using a Hamamatsu UV spot light source, generating a UV beam of $3500 \mathrm{~mW} / \mathrm{cm}^{2}$, with a radiant wavelength range from 240 to $400 \mathrm{~nm}^{2}$. The proper wavelength for ONB irradiation $(365 \mathrm{~nm})$ was selected using a set of UV filters (WG360 and UG11, Schott Glass), as shown in Figure 21.
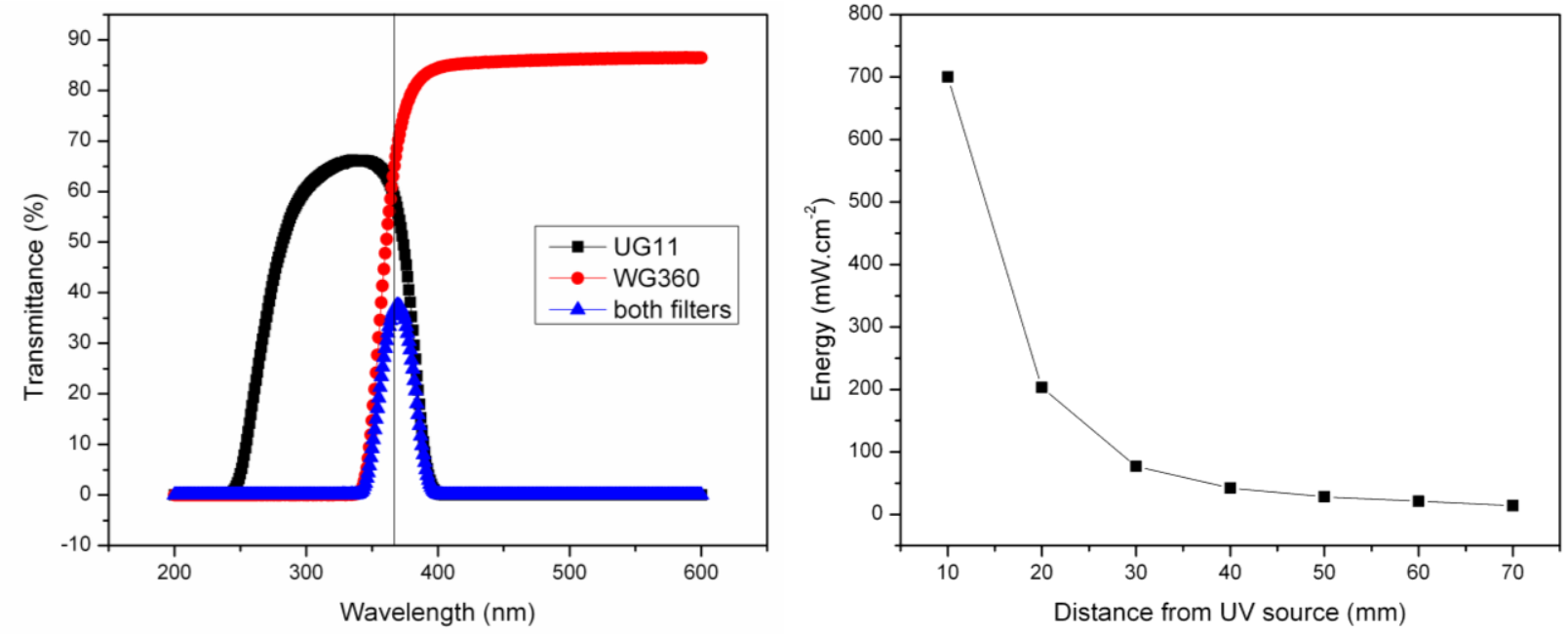

Figure 21: Left: UV transmittance with UV filters to isolate the $365 \mathrm{~nm}$ band. Right: estimated $U V$ intensity delivered to the samples as a function of distance from UV light source.

\footnotetext{
${ }^{2}$ To give an idea of UV energy scale, a few numbers are given here:

UVA + UVB solar radiation before atmosphere attenuation: $\sim 10 \mathrm{~mW} / \mathrm{cm}^{2} .118$. Frederick, J.E., H.E. Snell, and E.K. Haywood, SOLAR ULTRAVIOLET RADIATION AT THE EARTH'S SURFACE. Photochemistry and Photobiology, 1989. 50(4): p. 443-450.

UV solar radiation measured at noon (07.14.10), in the chemistry department courtyard of UniBasel,: $\sim 2 \mathrm{~mW} / \mathrm{cm}^{2}$ UV radiations used for water purification: 30 to $100 \mathrm{~mW} / \mathrm{cm}^{2}$
} 
The energy delivered to the sample was estimated from data provided by Hamamatsu, as a function of the distance in between the light output and the sample (Figure 21): at $1 \mathrm{~mm}$ the maximum UV energy used in this work was obtained $\left(700 \mathrm{~mW} / \mathrm{cm}^{2}\right)$, and the lowest energy used $\left(20 \mathrm{~mW} / \mathrm{cm}^{2}\right)$ at $6 \mathrm{~mm}$. A scheme of the UV setup is provided in Figure 22: the samples are placed in Quartz cuvettes to minimize UV absorption by common glass vials, at the desired distance from UV source.

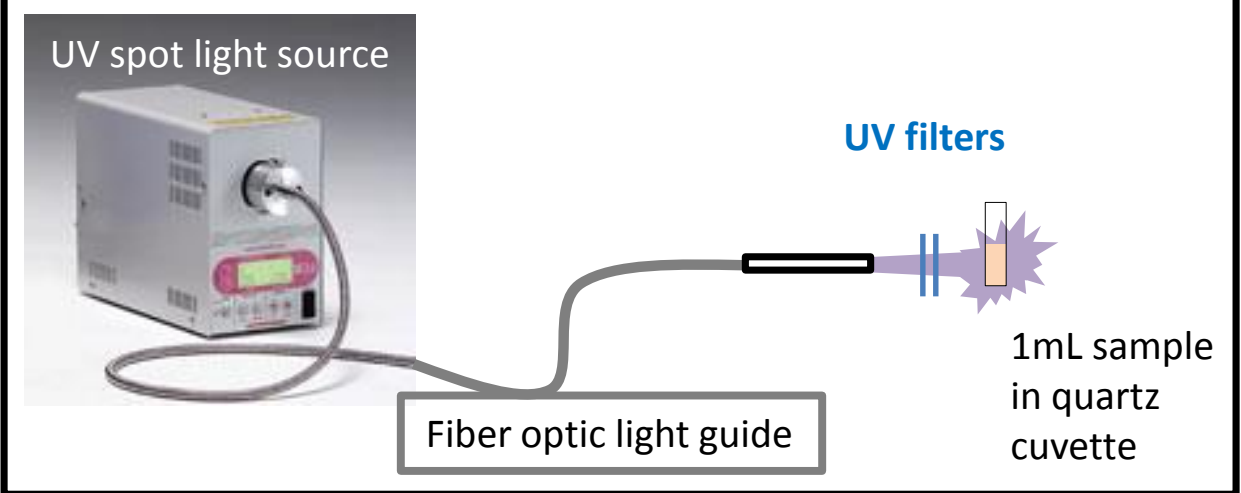

Figure 22: UV setup for UV irradiation experiments.

\subsection{Block copolymer photocleavage in solvent}

First, the cleavage of the block copolymers was investigated in solvents. To characterize the successful degradation of the linker, we followed the UV-Vis signal changes and the chemical modifications (with NMR spectroscopy) induced by UV irradiation. The subsequent separation of the two polymer segments was analyzed with GPC.

The degradation of O-nitrobenzyl moieties is well characterized. As mentioned before, it is an intramolecular process, i.e. it does not involve other chemical entities. Although the photochemistry of this compound is beyond the scope of this thesis, a schematic of its degradation, proposed by Blanc et al. ${ }^{[119]}$ is given in Figure 23. 


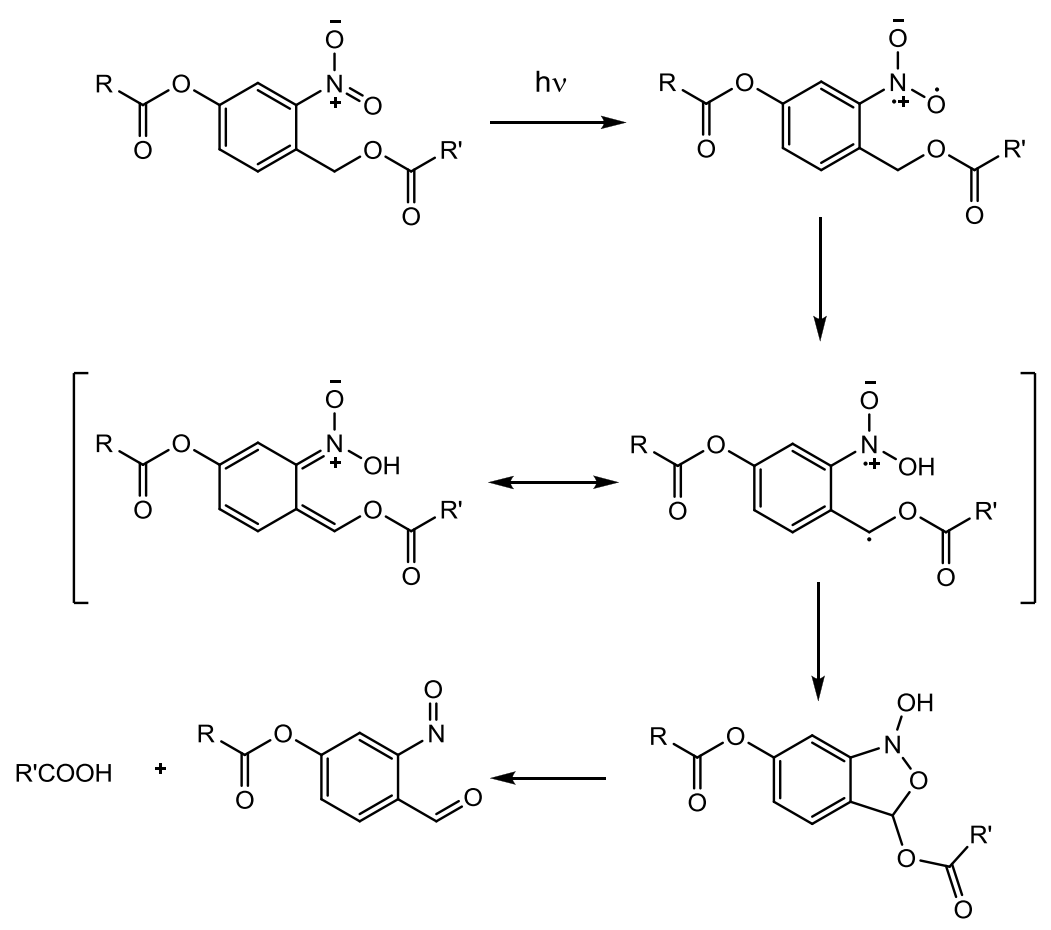

Figure 23: Degradation mechanism of the O-nitrobenzyl moiety, adapted from reference [119]. R: PAA block, and R': PMCL block.

According to this degradation mechanism, the rearrangement leads to the formation of an aldehyde functionality (which stays attached to the PAA segment), and a carboxylic acid group (on the PMCL block). As we will see, the presence of the - $\mathrm{COOH}$ ionisable group in the terminal positions of cleaved PMCL chains is playing a significant role in the degradation mechanism of the supramolecular structures.

\subsubsection{ONB photodegradation characterized by UV-Vis spectroscopy}

We investigated the photolytic degradation of the linker with UV-Vis spectroscopy, observing the spectral changes from a solution of copolymer over irradiation time. For this purpose we dissolved $\mathrm{PMCL}_{105}-\mathrm{ONB}-\mathrm{PAA}_{30}$ in inhibitor-free tetrahydrofuran (THF) (or dichloromethane, DCM) in quartz cuvettes, and irradiated the solutions with UV light ( 365 nm, 
$700 \mathrm{~mW} / \mathrm{cm}^{2}$ ) for a given amount of time. The UV-Vis spectra (Figure 24) show a clear shift over irradiation time.
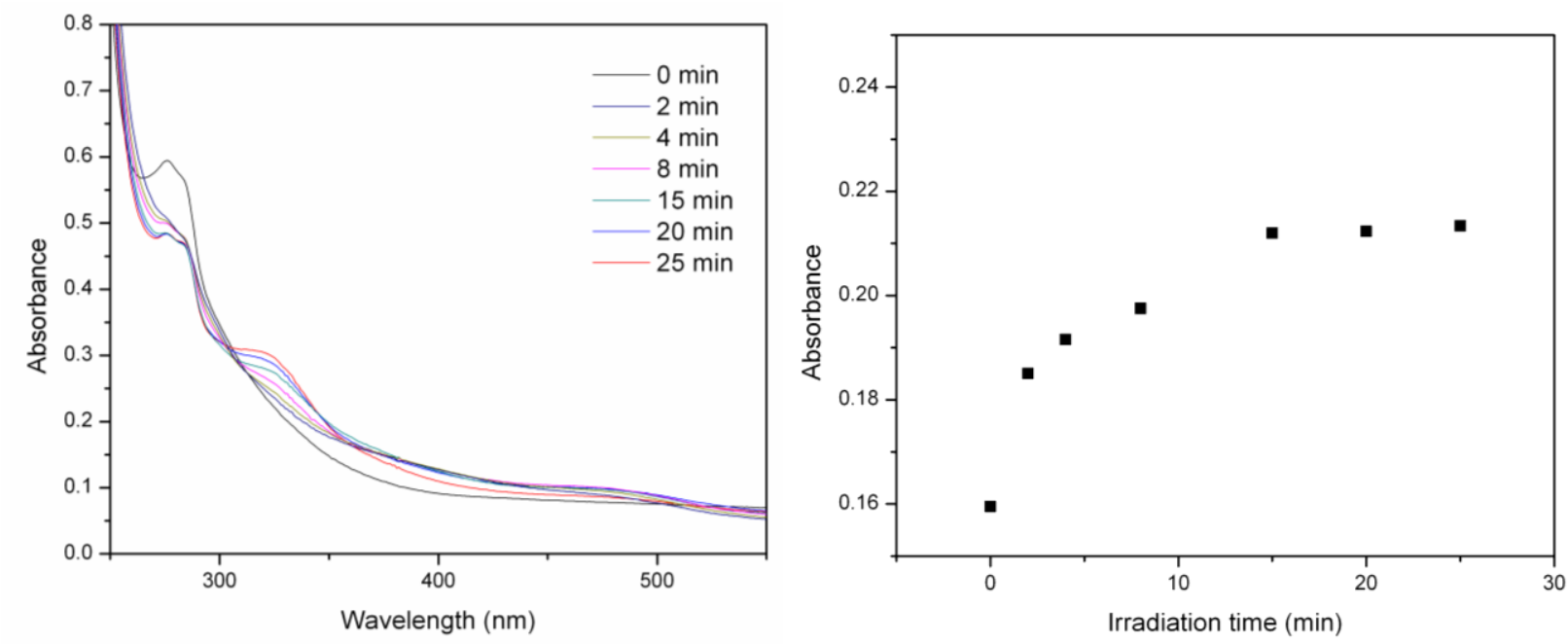

Figure 24: UV traces of PMCL-ONB-PtBA block copolymers after different irradiation times. Left: full spectra in DCM, right: absorbance increase at $\lambda=345 \mathrm{~nm}$. Irradiation: $700 \mathrm{~mW} / \mathrm{cm}^{2}$, with $\lambda=365 \mathrm{~nm}$.

The spectral changes reflect the typical red-shifted absorbance (bathochromic shift) observed for the transformation of the nitrobenzyl linker to the nitrosobenzaldehyde compound, and therefore points toward the photolysis of the ONB linker. ${ }^{[120]}$ It should be emphasized that the complete degradation of the linker is reached after only 20 minutes of irradiation. This can be understood by the increase of the intensity of the UV-Vis band at $345 \mathrm{~nm}$ as function of time, up to complete degradation (Figure 24). To prove that the bathochromic shift is solely resulting from the linker photodegradation, a control experiment was performed, where the UV-Vis spectra of a PMCL $38-\mathrm{PtBA}_{11}$ copolymer with no photocleavable linker were recorded over UV irradiation time. The spectra are given in Figure 25, and show that no red shift is observed in the region of interest, due to the absence of the ONB photolinker. 


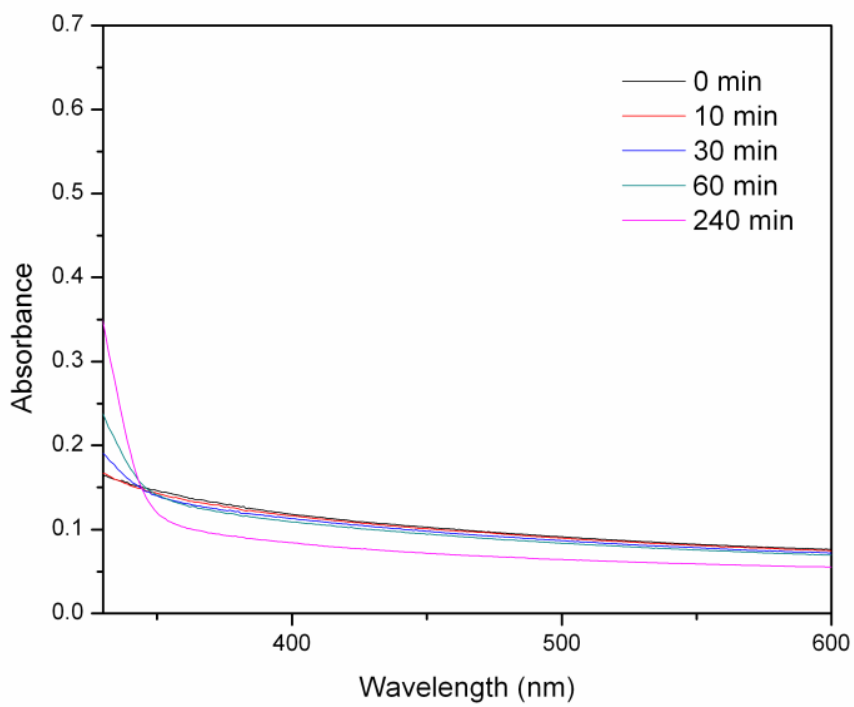

Figure 25: UV-Vis spectra of a PMCL-PtBA block copolymer inert toward UV irradiation. Irradiation: $700 \mathrm{~mW} / \mathrm{cm}^{2}, \lambda=365 \mathrm{~nm}$.

\subsubsection{ONB photodegradation followed by ${ }^{1} \mathrm{H}-\mathrm{NMR}$ spectroscopy}

Additionally, the photodegradation was followed by ${ }^{1} \mathrm{H}$ NMR. In Figure 26, significant changes in chemical shifts are observed. As described previously (Figure 23), the ester linkage between ONB and PMCL is converted to an aldehyde functionality upon degradation.

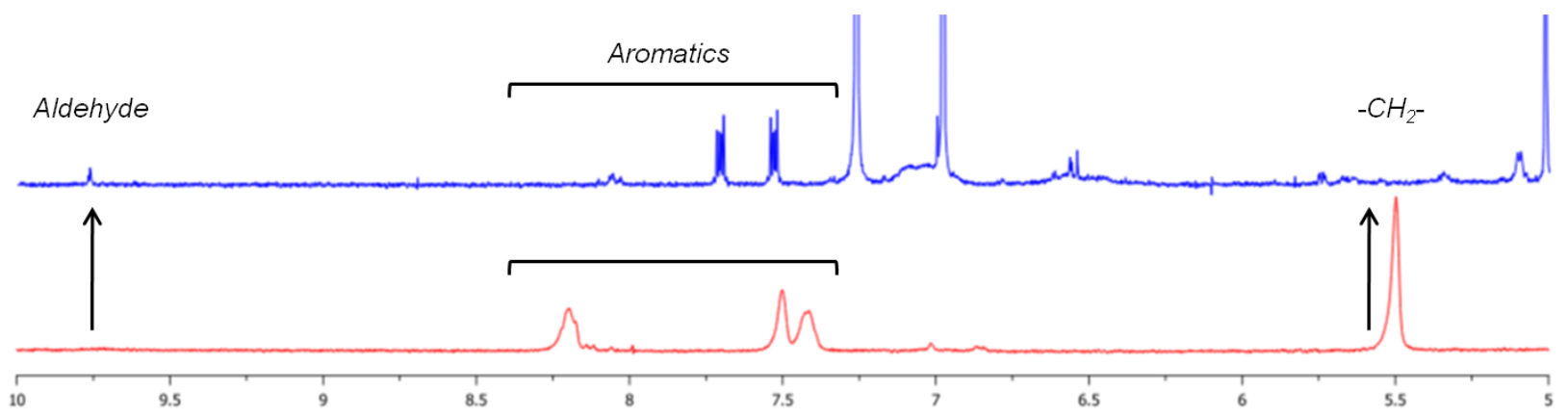

Figure 26: ${ }^{1} H$ NMR of a PMCL-ONB-PAA before UV irradiation (red), and after UV-induced cleavage (blue). 
On the ${ }^{1} \mathrm{H}-\mathrm{NMR}$ spectra, the $\mathrm{CH}_{2}$ signal at 5.5 ppm disappears and a peak corresponding to aldehydes appears (around $9.8 \mathrm{ppm}$ ), indicating that the photolysis took place. In addition, the aromatic signals are also affected: the ortho proton $\mathrm{d}$ is shifted from $\delta=8.2 \mathrm{ppm} \delta \approx 7.0 \mathrm{ppm}$. The nitroso group formed upon UV degradation has a smaller deshielding effect when compared to the nitro group. Therefore, the signal is shifted toward lower ppm values (higher fields). The effect is clearly visible on ortho protons, but less pronounced with meta and para protons. ${ }^{[121]}$

\subsubsection{GPC analysis: evidence of block cleavage}

To further prove the degradation of the copolymer into its two constituting blocks, the UV exposed polymer was analyzed with GPC. After UV irradiation, the copolymer peak is no longer observed, and the chromatogram shows the appearance of two new peaks (Figure 27). The main peak corresponds to the cleaved PMCL: its retention volume is slightly higher than the retention time of the diblock, indicating a decrease in molecular weight. As for the second peak, it represents the low molecular weight cleaved PAA chains. The intensity of this peak is extremely small when compared to the peak associated to PMCL cleaved chains. It can be explained both by the poor solubility of free PAA chains in THF, and by the weight proportion of the PAA vs. PMCL in the copolymer.

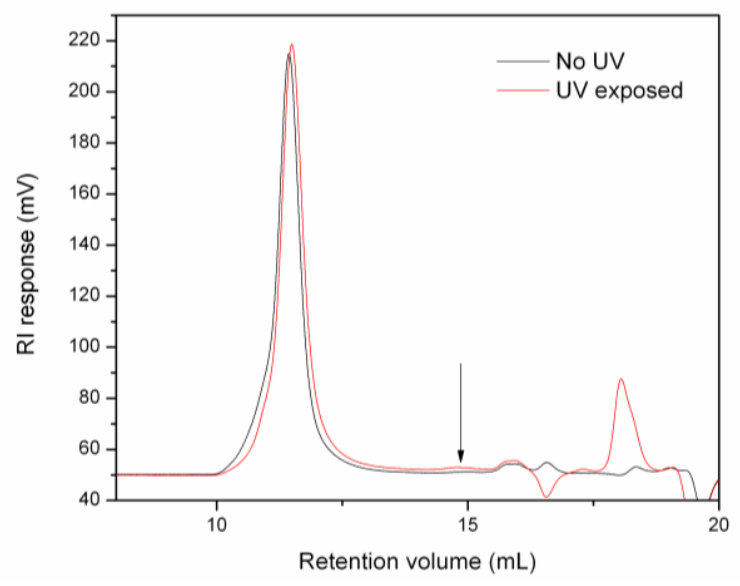

Figure 27: Refractive index (RI) response chromatograms of a PMCL-ONB-PAA copolymer before and after UV irradiation. 
However, this second peak can be visualized more easily when considering the UV signal obtained by GPC (Figure 28). In this second chromatogram, the high UV intensity of the PAA peak is due to the O-nitrosobenzene residue attached to the PAA chains after degradation (see Figure 23). The presence of this new peak associated to low molecular weight polymer chains with high UV absorbance, reveals the successful cleavage of the copolymer.
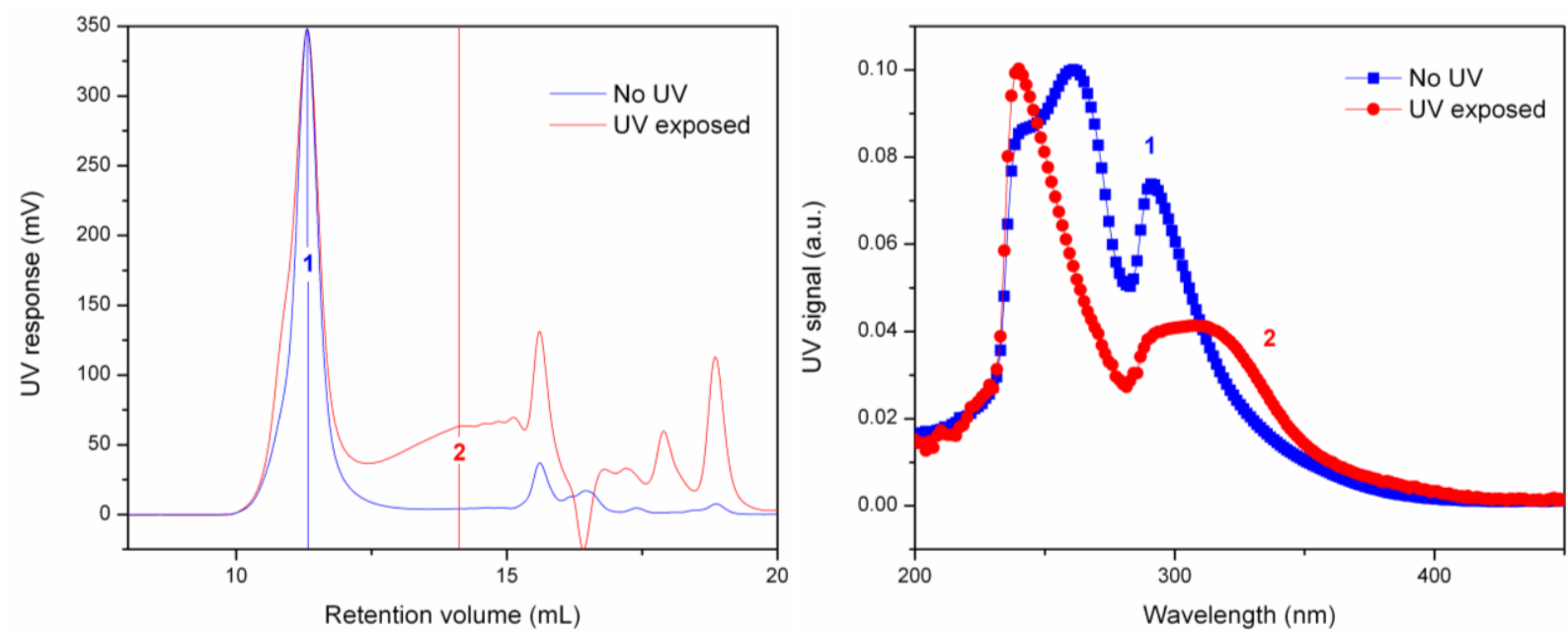

Figure 28: (Left )UV response chromatograms of a PMCL-ONB-PAA copolymer before and after UV irradiation, showing the formation of small molecular weight entities (2).(Right) $U V$ spectra of single retention volume 1 and 2 illustrating the red-shifted absorption of the degraded linker attached to low Mw PAA segments.

With the Viscotek GPC setup, it is possible to acquire the UV-Vis spectrum for a given volume fraction. Figure 28 shows the UV-Vis spectrum of the copolymer prior to UV exposure and the UV-Vis spectrum of the cleaved PAA fraction (retention volumes respectively labeled 1 and 2). These spectra show the significant red-shifted absorption associated with the degradation of the ONB linker to the O-nitrosobenzene residue (fraction 2) when compared with the intact copolymer (fraction 1), thus confirming the UV-induced cleavage of the copolymer. 


\subsection{Block copolymer photocleavage in water}

The cleavage of PMCL-ONB-PAA in aqueous environment was investigated similarly to solvent environment. Our aim was to prove that, despite this dramatic environmental change, the copolymer can still be efficiently cleaved.As stated before and described in detail in the next chapter, PMCL-ONB-PAA copolymer are self-assembling when hydrated, due to their amphiphilic nature. Solutions of polymer aggregates $(1 \mathrm{mg} / \mathrm{mL})$ in phosphate buffered saline (PBS, pH 7.4) were prepared. In the supramolecular structures formed, the polymer chains adopt new conformations (from random coil in solvent to stretched in membrane) and organize into ordered structures (interdigitated polymer membranes). As a result, the environment around the ONB linker is completely different: in organic solvent it is surrounded by solvent molecules, and in supramolecular structures it sits at the water-polymer interface in a segregated microdomain.

\subsubsection{UV-Vis spectroscopy}

When the solution of self-assembled structures is exposed to UV, a clear color change is observed, along with the red shifted absorption (max. at $\lambda=340 \mathrm{~nm}$ ) observed in experiments performed in solvent solutions (Figure 29).

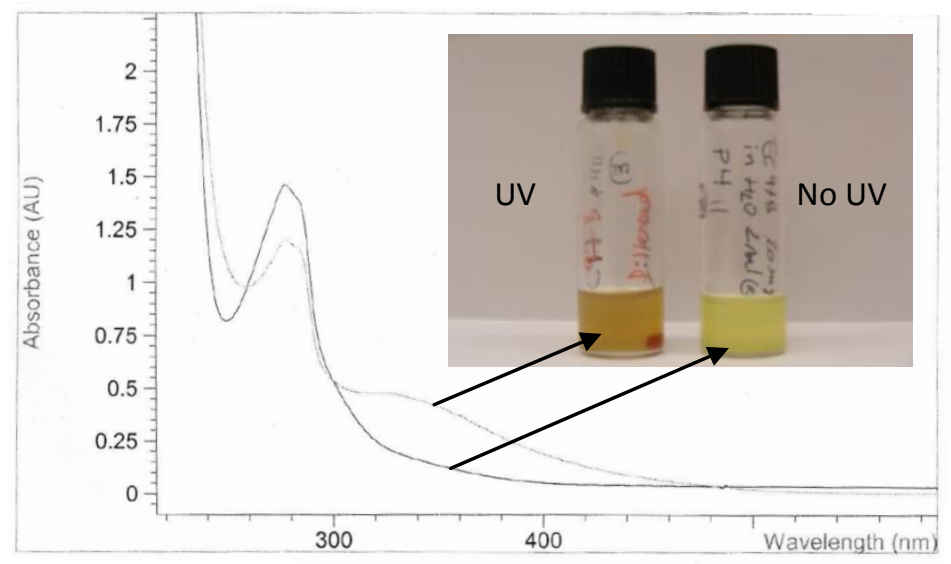

Figure 29: UV exposed PMCL-ONB-PAA solution in PBS and UV-Vis spectra associated. 
As shown in Figure 30, the absorbance in the red region is increasing with irradiation time, indicating that the ONB linker is progressively degraded. It is interesting to note that, at constant UV energy $\left(700 \mathrm{~mW} / \mathrm{cm}^{2}\right)$, the UV exposure times to achieve cleavage of the polymer both in organic solvent and aqueous environment are very similar ( $20 \mathrm{~min})$. This result is quite consistent with the fact that the ONB linker photodegradation is an intramolecular rearrangement (i. e. it is rather independent of the environment in which the photodegradation occurs). ${ }^{[64,119]}$ Hence the linker is degraded in a similar fashion, whether it sits in solvent or in aqueous environment.
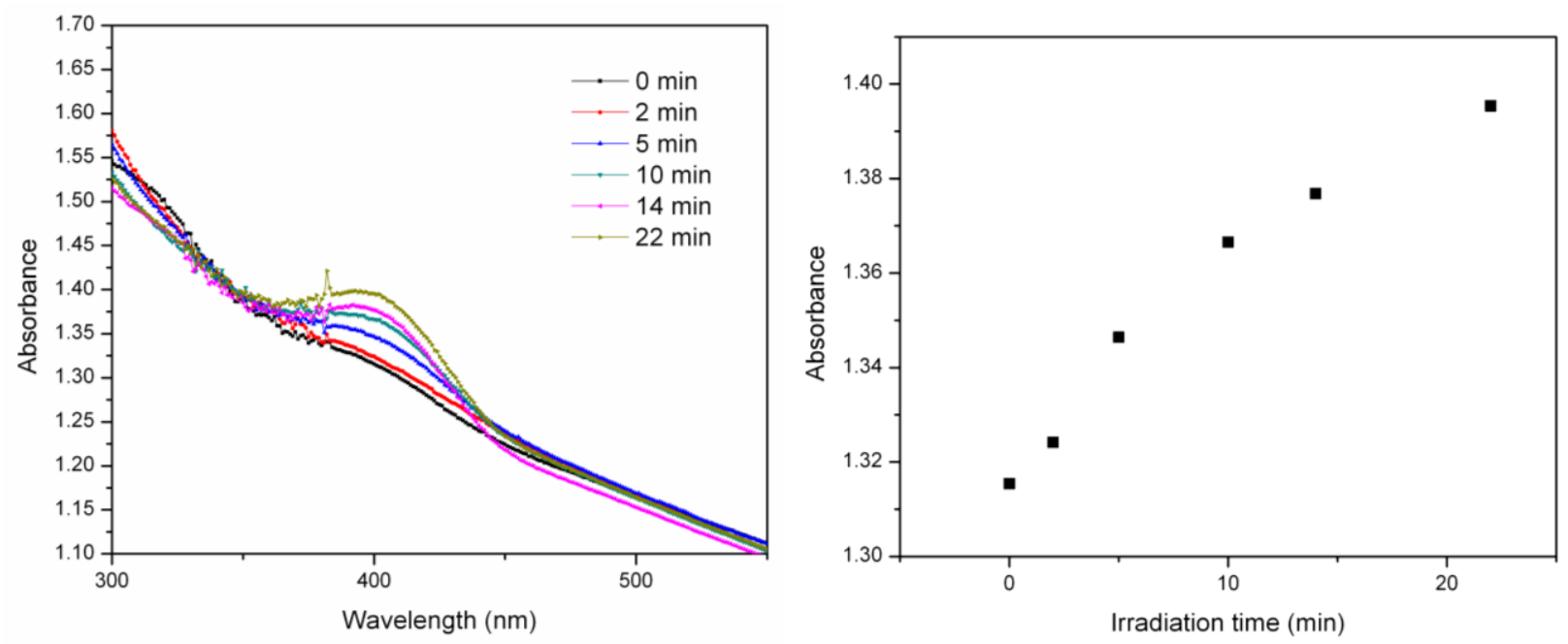

Figure 30: UV vis spectra of polymer degradation in PBS (left), and absorbance increase at $\lambda=$ 400nm vs. irradiation time (right). Irradiation: $700 \mathrm{~mW} / \mathrm{cm}^{2}, \lambda=365 \mathrm{~nm}$.

\subsection{2 $\quad{ }^{1}$ H-NMR spectroscopy}

In an effort to give further proof of the photoinduced cleavage of the copolymer in aqueous solutions, the UV-exposed polymer segments were isolated, and analyzed separately with ${ }^{1} \mathrm{H}$ NMR. After UV irradiation of a copolymer solution in PBS, the hydrophobic cleaved PMCL chains were extracted from PBS solution with dichloromethane, a good solvent for hydrophobic polymers, and a poor solvent for hydrophilic charged polymers (such as PAA). The 
NMR spectra of the PMCL-ONB macroinitiator, the intact PMCL-ONB-PAA copolymer, and the extracted cleaved PMCL segments are given in Figure 31.

A
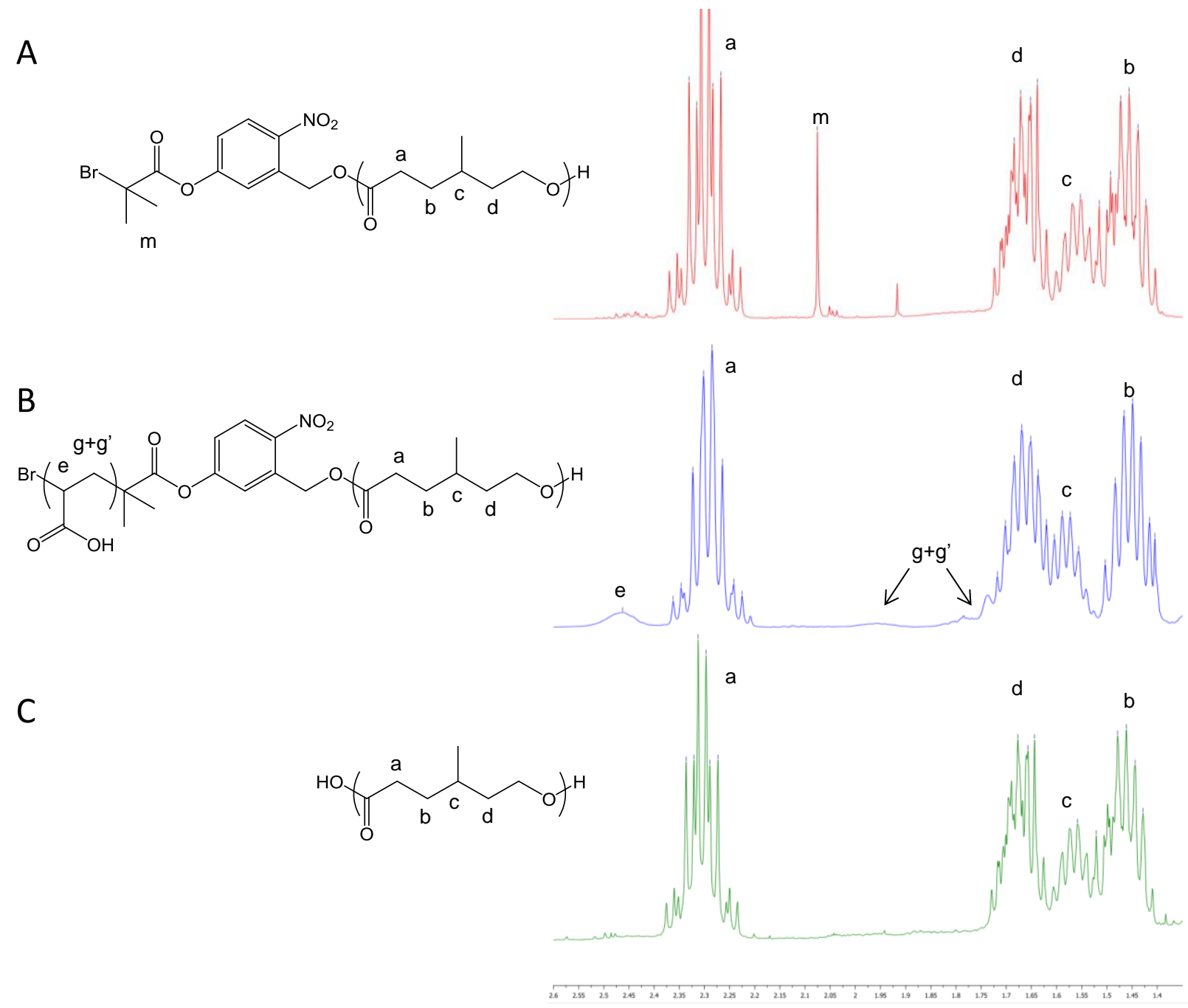

Figure 31: ${ }^{1} \mathrm{H} N M R$ spectra of: (A) $\mathrm{PMCL}_{30} \mathrm{ONB}$ macroinitiator in $\mathrm{CDCl}_{3}$; (B) $\mathrm{PMCL}_{30^{-}} \mathrm{ONB}$ $\mathrm{PAA}_{5}$ in $\mathrm{THF}-d_{8}$, and $(C) \mathrm{PMCL}_{30}$ block in $C D C l_{3}$ cleaved by $U V$ irradiation. The shift observed for the c peak is due to the different solvents used. Irradiation: $700 \mathrm{~mW} / \mathrm{cm}^{2}, \lambda=365 \mathrm{~nm}$.

As shown in spectrum $\mathrm{C}$ for cleaved polyester chains, the PAA peaks (see spectrum B, peaks e, $g$ and g') are not present anymore, indicating that the extracted block is purely PMCL, without copolymer. In addition, the methyl protons characterizing the tertiary bromine end of the 
PMCL-ONB macroinitiator (spectrum A, m signals) are not seen in the cleaved PMCL spectrum. This is another indication that the ONB residue stays attached to the PAA block upon cleavage, and it proves that spectrum $\mathrm{C}$ represents a cleaved PMCL, and not a non-polymerized macroinitiator.

\subsubsection{Further degradation}

Surprisingly, when the irradiation is continued (i.e. up to 2 hours or more), the red-shifted band observed in UV-Vis spectra starts to decrease (Figure 32).
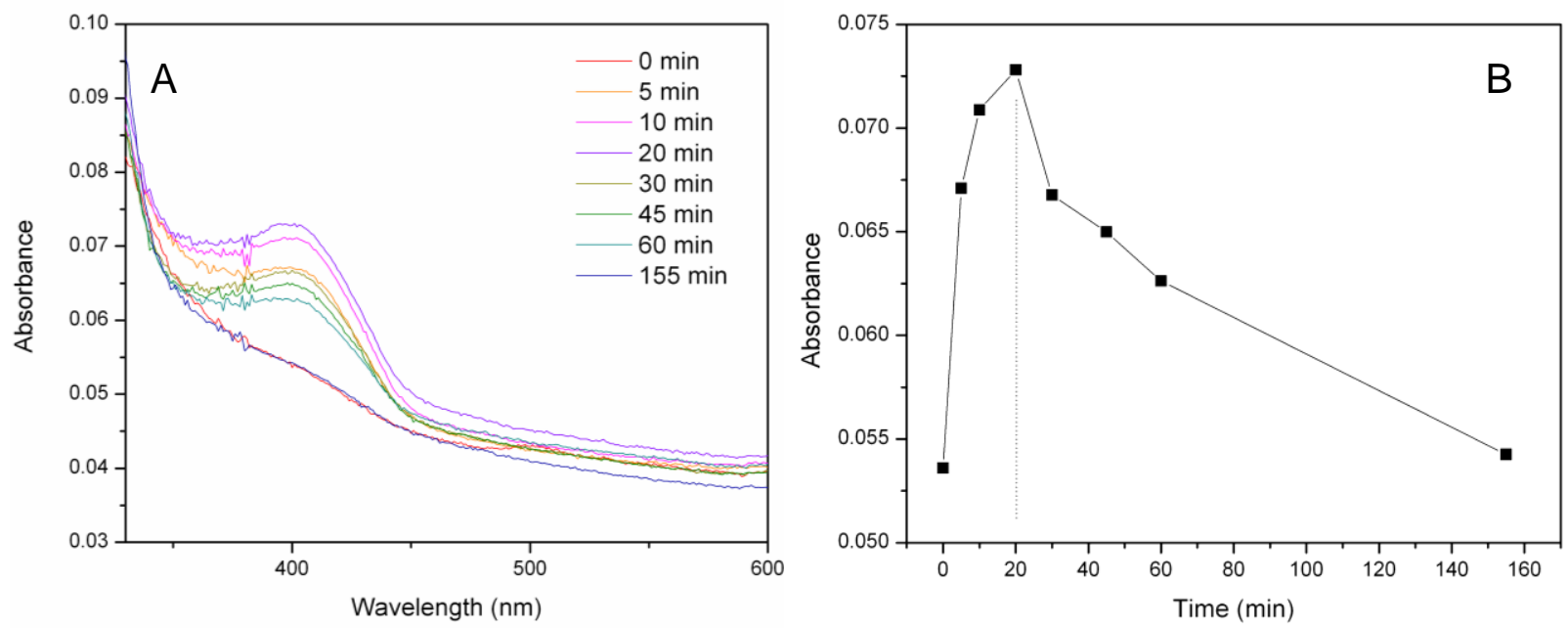

Figure 32: Prolonged irradiation time of a PMCL-ONB-PAA solution in PBS. (A) UV-Vis spectra recorded over time showing a progressive decrease of the absorbance after 20 minutes of $U V$ exposure; (B) Absorbance at $\lambda=400 \mathrm{~nm} v$ s. irradiation time. Irradiation: $700 \mathrm{~mW} / \mathrm{cm}^{2}, \lambda$ $=365 \mathrm{~nm}$.

The photochemistry of ONB structures is a complex phenomenon. The aldehyde group created upon UV irradiation is quite reactive and, according to literature, the nitrosobenzene compound can further react in the presence of a base, or with further exposition to UV-light. ${ }^{\text {[122] }}$ 
It then eventually results into the coupling of two nitrosobenzene structures to form a bis-aryl compound as described in Figure 33.

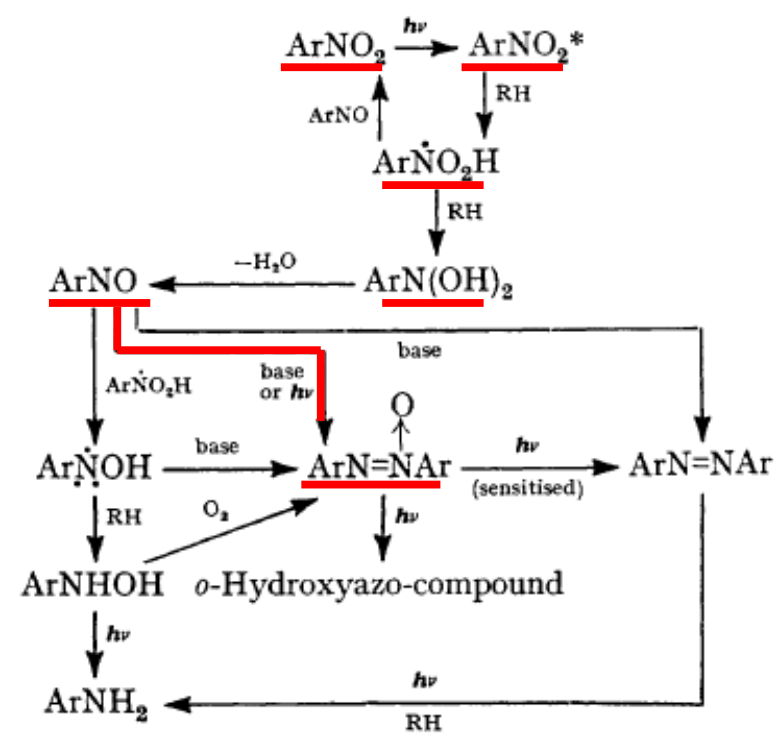

Figure 33: Degradation scheme of nitrobenzene species as proposed by Barltrop et al. ${ }^{[122]}$

In our case, the aldehyde functionality is actually a chain end-group on the PAA chain. Therefore the coupling of two entities would result in the formation of a long PAA chain. Interestingly, GPC measurements performed on a $\mathrm{PCL}_{55}-\mathrm{ONB}-\mathrm{PAA}_{10}$ copolymer after long exposure times, give further evidence to this hypothesis. In these chromatograms (Figure 34), a high molecular weight peak can be seen (with all detectors, i.e. refractive index (RI), UV-Vis, right angle light scattering (RALS), and viscosity). According to GPC calculations, this peak corresponds to a fraction with a Mw of $90000 \mathrm{~g} / \mathrm{mol}$. The copolymer itself has a total molecular weight of $10000 \mathrm{~g} / \mathrm{mol}$. In the case of coupled PAA chains, their molecular weight would reach a maximum of $2000 \mathrm{~g} / \mathrm{mol}$. Therefore, the high molecular weight fractions are probably resulting from aggregation of several polymer chains.

In this respect, it is interesting to see that in RALS signal, the fraction corresponding to this high Mw peak scatters more than the other fractions. 

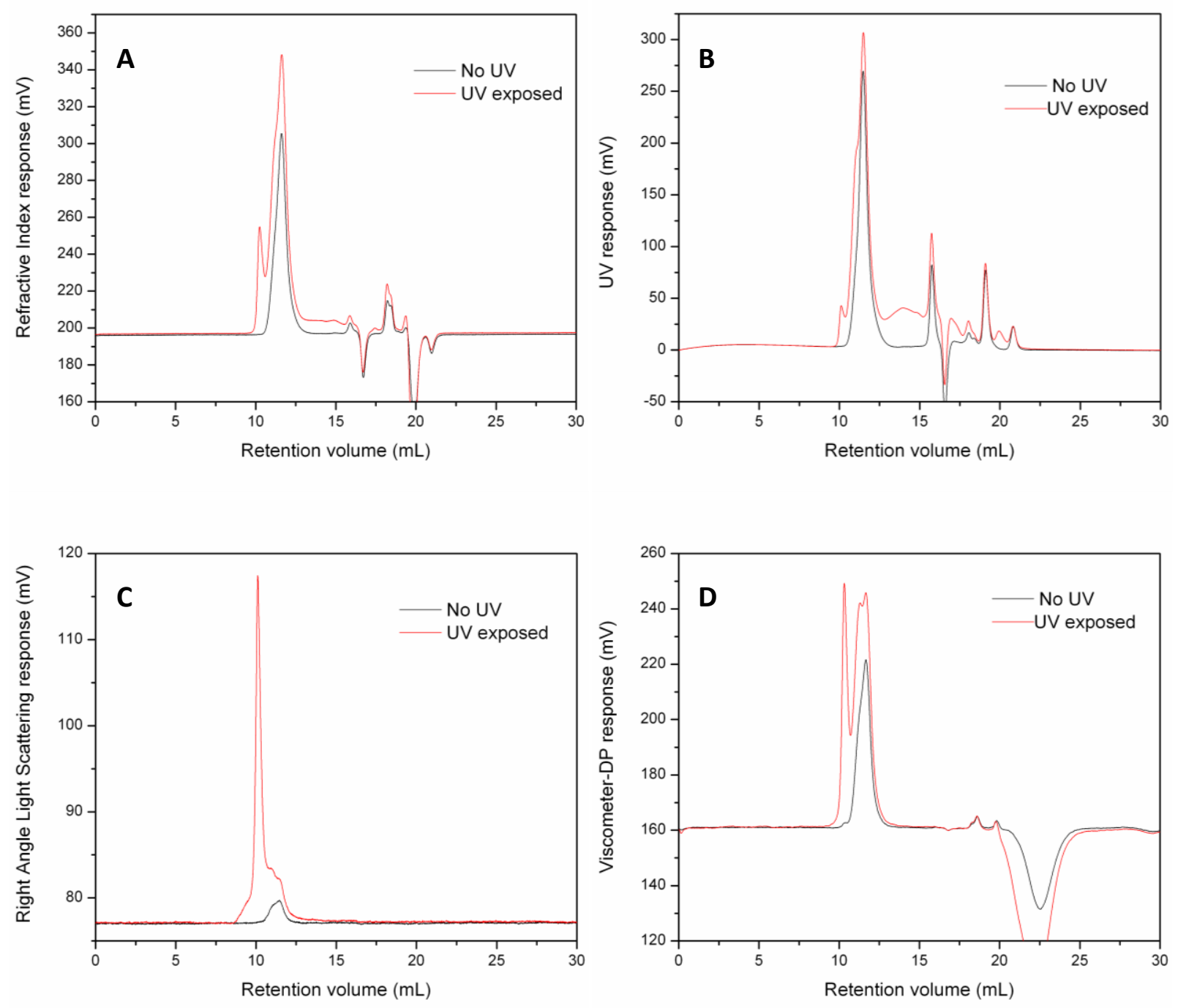

Figure 34: GPC chromatograms of PCL-ONB-PAA before and after UV degradation: (A) refractive index, (B) $U V$-Vis, (C) light scattering, (D) viscometry. Irradiation: $700 \mathrm{~mW} / \mathrm{cm}^{2}, \lambda=$ $365 \mathrm{~nm}$.

From the light scattering theory, we know that large objects scatter more. Therefore, consistently with the $\mathrm{Mw}$ calculated, we assume that this fraction is actually due to small aggregates rather than fully dissolved polymer chains in a coiled conformation. One explanation could be that these aggregates are constituted by long PAA chains resulting from coupling of two PAA segments via the ONB rearrangement. The formation of aggregates is plausible since newly 
created PAA chains are even less soluble than shorter PAA chains. This hypothesis is reinforced by the viscosity measurement from GPC: the high viscosity of the high molecular weight peak is due to these aggregates, behaving as "beads" in a fluid, which, from a rheological point of view, induce an increase of the viscosity.

As a conclusion, and according to all characterization techniques used (UV-Vis, NMR, and GPC), the PMCL-ONB-PAA copolymer is efficiently cleaved upon UV-irradiation, both in solvent and in aqueous solution, and yields two separated homopolymers, PMCL and PAA. 


\section{Self-assembled structures and their UV-triggered degradation}

In a first part, different supramolecular structures generated by the self-assembly of PMCL-ONB-PAA (and PCL-ONB-PAA) copolymers in aqueous solutions, are characterized by dynamic light scattering (DLS), static light scattering (SLS), and transmission electron microscopy (TEM). In a second part, the changes in size and morphology of the self-assembled superstructures induced by UV-irradiation are described. Based on these results, the third section suggests a plausible degradation mechanism at molecular level.

\subsection{Self-assembly of PMCL-ONB-PAA and PCL-ONB-PAA copolymers in PBS}

We investigated the formation of self- assembled structures of block copolymer PMCL$O N B$-PAA using the co-solvent method. The copolymers were dissolved in a minimum amount of solvent (THF), and buffer solution (PBS, pH 7.4) was slowly added, to reach a typical polymer concentration of $1 \mathrm{mg} / \mathrm{mL}$. When needed, the $\mathrm{pH}$ was adjusted to 7.4 with $0.5 \mathrm{M} \mathrm{NaOH}$ solution. The solutions were stirred for 3 days, allowing the system to equilibrate, and the small amount of solvent to evaporate.

\subsubsection{Preliminary studies with PCL-ONB-PAA and PMCL-ONB-PAA}

The molecular weights and $f$ ratios of copolymer used in this study are given in Table 4 .

Table 4: Characteristics of copolymers used for self-assembly.

\begin{tabular}{|c|c|c|}
\hline Polymer & $\mathrm{M}_{\mathrm{n}}(\mathrm{g} / \mathrm{mol})^{\mathrm{a}}$ & $f^{\mathrm{b}}$ \\
\hline $\mathrm{PCL}_{55}-\mathrm{ONB}-\mathrm{PAA}_{8}$ & 7200 & 0.09 \\
\hline $\mathrm{PCL}_{55^{-}} O N B-\mathrm{PAA}_{30}$ & 8900 & 0.26 \\
\hline $\mathrm{PCL}_{55}-O N B-\mathrm{PAA}_{10}$ & 7400 & 0.11 \\
\hline
\end{tabular}




\begin{tabular}{|c|c|c|}
\hline $\mathrm{PMCL}_{55}-O N B-\mathrm{PAA}_{10}$ & 8100 & 0.1 \\
\hline $\mathrm{PMCL}_{23}-O N B-\mathrm{PAA}_{20}$ & 4800 & 0.32 \\
\hline $\mathrm{PMCL}_{30}-O N B-\mathrm{PAA}_{6}$ & 4600 & 0.1 \\
\hline $\mathrm{PMCL}_{30^{-}} O N B-\mathrm{PAA}_{10}$ & 4900 & 0.16 \\
\hline $\mathrm{PMCL}_{105}-O N B-\mathrm{PAA}_{30}$ & 16100 & 0.15 \\
\hline $\mathrm{PMCL}_{150^{-}} O N B-\mathrm{PAA}_{10}$ & 20300 & 0.04 \\
\hline $\mathrm{PMCL}_{150^{-}} O N B-\mathrm{PAA}_{23}$ & 21350 & 0.08 \\
\hline $\mathrm{PMCL}_{76}-O N B-\mathrm{PAA}_{41}$ & 13250 & 0.24 \\
\hline $\mathrm{PMCL}_{76}-\mathrm{ONB}-\mathrm{PAA}_{16}$ & 11550 & 0.11 \\
\hline $\mathrm{PMCL}_{76}-\mathrm{ONB}-\mathrm{PAA}_{8}$ & 10650 & 0.06 \\
\hline
\end{tabular}

${ }^{a}$ Number-average molecular weight of block copolymers as determined by ${ }^{1} \mathrm{H}-\mathrm{NMR}$ spectroscopy. ${ }^{\mathrm{b}}$ Hydrophilic to hydrophobic weight ratio.

We used TEM to visualize aggregates formed upon self-assembly, and characterize their morphologies. A variety of structures can be formed with PCL-ONB-PAA and PMCL-ONBPAA diblocks, including micelles and vesicles.

Vesicles formed by PCL containing blocks have been reported before, especially with PEG-PCL diblocks, ${ }^{[123,124]}$ but only micelles were described using PCL-PAA copolymers. Indeed, with a copolymer PCL $_{55}-O N B-\mathrm{PAA}_{30}$ with a $f$ ratio of 0.26 , TEM micrographs revealed the presence of micelles (Figure 35, A and B). Those spherical structures are constituted by a PCL rich core and a PAA corona. 

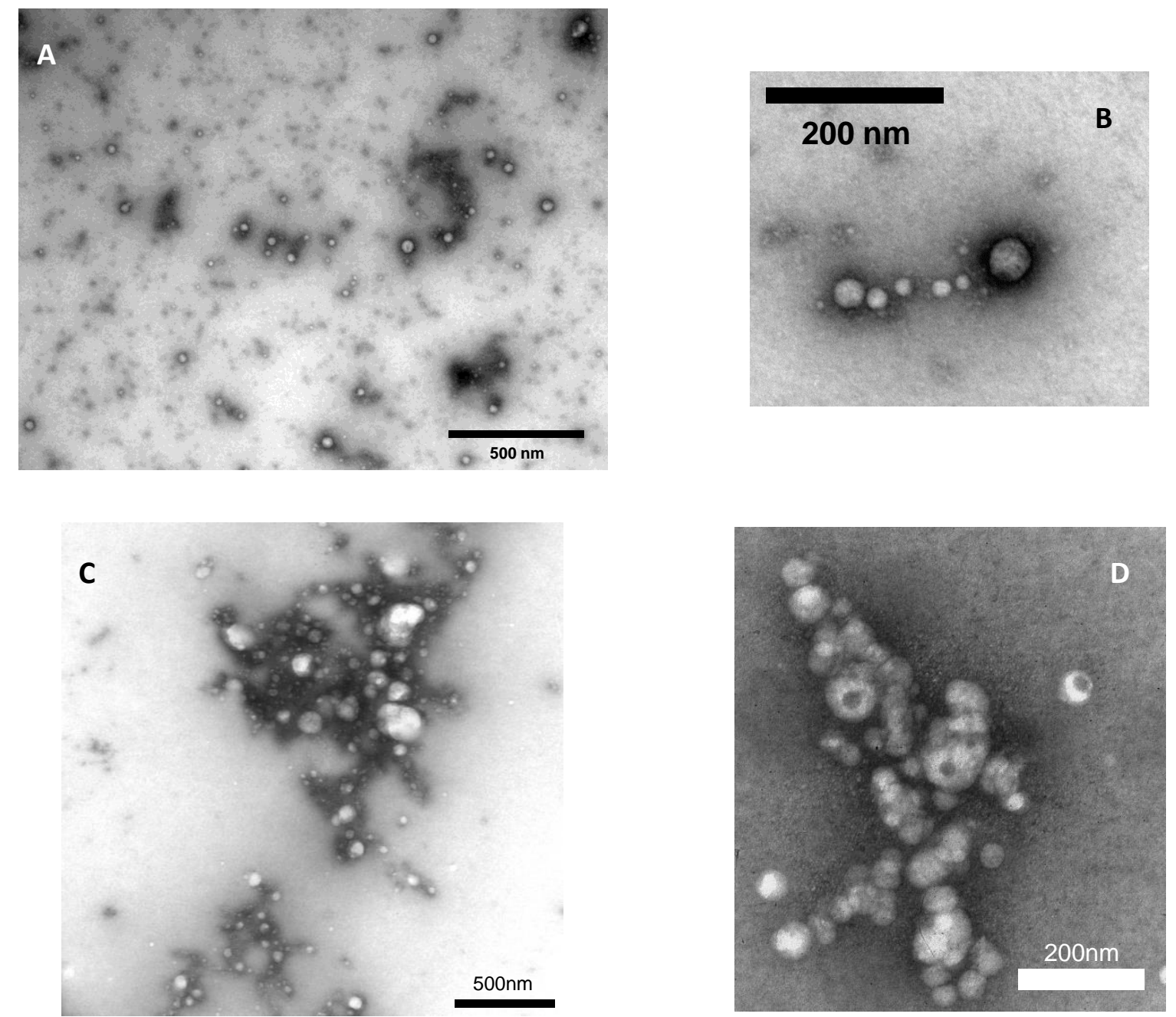

Figure 35: TEM pictures of superstructures obtained by self-assembly in PBS of: $(A)$ and (B) $P C L_{55}-O N B-P A A_{30}$; (C) and (D) PMCL $L_{76}-O N B-P A A_{41}$.

When a PMCL-ONB-PAA copolymer with similar $f$ ratio was used (PMCL $_{76}-\mathrm{ONB}$ $\mathrm{PAA}_{41}, f=0.25$ ), we observed aggregated clusters (Figure 35, C and D). The aggregation contrasts with the well defined and isolated micelles observed with the PCL based block copolymer. Presumably, the glassy nature of PCL provides stiffness to the micelles. Upon drying, the structures keep their shapes, and the electrostatic repulsions between PAA coronas are sufficient to prevent aggregation. But in the case of PMCL based self-assemblies, the flexible 
polymer imparts fluidity to the structures, which collapse completely upon drying, forming aggregates.

Dynamic light scattering provides information on size and size distribution of particles in solution. The size distribution histograms obtained by DLS confirm the presence of micelles for both copolymers, with radii around $15 \mathrm{~nm}$ for $\mathrm{PCL}_{55}-\mathrm{ONB}-\mathrm{PAA}_{30}$ and $30 \mathrm{~nm}$ for $\mathrm{PMCL}_{76}-\mathrm{ONB}$ PAA $_{41}$ (Figure 36). In PBS solution, the highly charged PAA corona chains are in a fully stretched conformation, due to charge repulsions inducing chain expansion. ${ }^{[125]}$ Therefore, the difference in size observed for the two copolymers may be explained by the length difference in between PAA chains. The histogram also reveals the presence of larger particles in the case of $\mathrm{PMCL}_{76^{-}} \mathrm{ONB}-\mathrm{PAA}_{41}$, which we assume to correspond to the micellar aggregates observed with TEM.

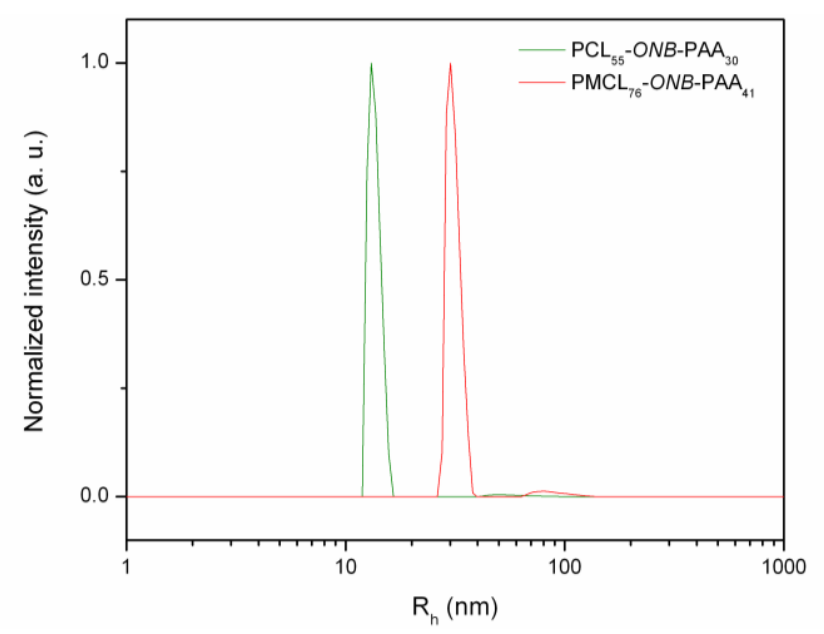

Figure 36: Typical DLS histograms for diblock copolymers with high f ratios. Number-averaged size distributions, $90^{\circ}$ measurements, CONTIN fit.

\subsubsection{Theory on $f$ ratio and polyelectrolytes}

The morphology and size of self-assembled structures is highly depending on the $f$ ratio. ${ }^{[8]}$ If the hydrophilic block is long with respect to the hydrophobic block, then the large volume occupied by the chains on the outer part is driving the self-assembly toward a highly 
curved structure, such as a micelle. With the size of the hydrophilic block decreasing (at a constant hydrophobic block length), aggregate's shape may evolve to rod-like micelles, and finally to vesicles. ${ }^{[126]}$

The influence of the $f$ ratio may actually be even more pronounced for the class of polymers used in this work, i.e. charged polymers. Due to electrostatic interactions, hydrophilic charged blocks are bulkier than commonly used blocks such as PEO. The corona forming polymer chains require a larger volume to minimize the strong electrostatic repulsions in between charges. Hence, such copolymers are more prone to form smaller objects with high curvature radii, i.e. micelles. ${ }^{[34]}$

Therefore, by shortening the lengths of hydrophilic blocks relative to the hydrophobic blocks we tried to favor the formation of vesicles.

\subsubsection{Polymers with low $f$ ratios forming vesicles}

With copolymers of lower $\mathrm{f}$ ratio $\left(\mathrm{PCL}_{55}-\mathrm{ONB}-\mathrm{PAA}_{10}, f=0.11 ; \mathrm{PMCL}_{105}-\mathrm{ONB}-\mathrm{PAA}_{30}, f\right.$ $=0.15 ; \mathrm{PMCL}_{30}-O N B-\mathrm{PAA}_{6}, f=0.1$ ), micelles are still observed in large proportions. However, the vesicles with diameters around 200nm were also revealed (Figure 37). According to the TEM micrographs, the amount of vesicles (versus micelles) is significantly higher in these samples, when compared to copolymers with $f$ ratios around 0.25 . It is interesting to note that, due to the high glass transition temperature $\left(\mathrm{T}_{\mathrm{g}}\right)$ of PCL and its crystalline nature, most of the observed vesicles are collapsed or even burst. 

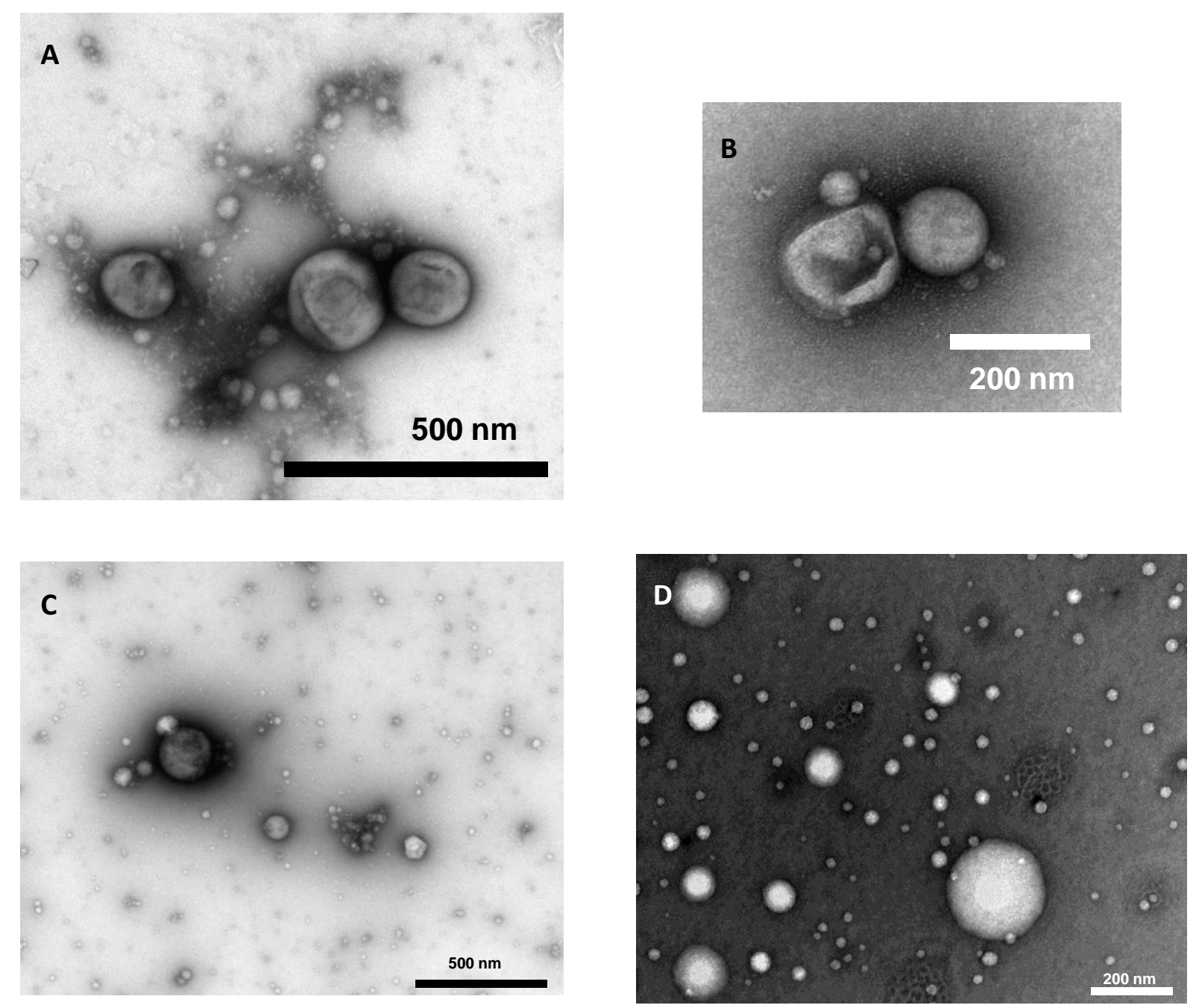

Figure 37: TEM micrographs showing vesicles and micelles from self-assembled copolymers in PBS: (A) and (B) $P C L_{55^{-}} O N B-P A A_{10}$; (C) PMCL ${ }_{105}-O N B-P A A_{30}$; (D) $P M C L_{30}-O N B-P A A_{6}$. 


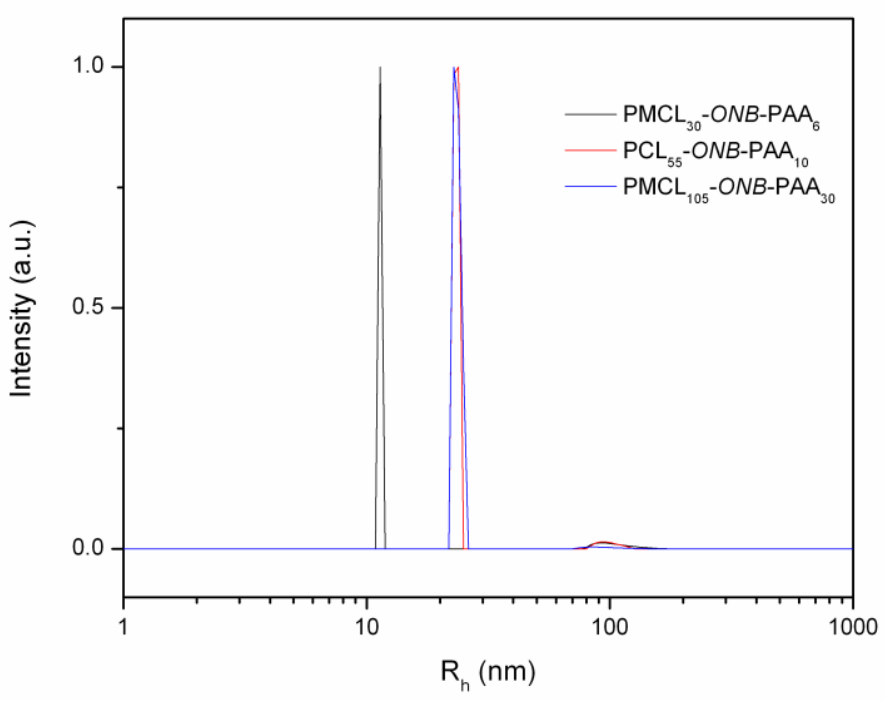

Figure 38: Typical DLS histograms for diblock copolymers with low $f$ ratios. Number-averaged size distributions, $90^{\circ}$ measurements, CONTIN fit.

DLS histograms for copolymers with lower $f$ ratios show two populations, micelles (with diameters around 10 and 20nm) and vesicles with diameters around 180nm. For the copolymers with the lower $\mathrm{f}$ ratios $(0.1$ and 0.11$)$, the peak associated to the polymersomes $\left(R_{h}=90 \mathrm{~nm}\right)$ accounts for 3 to $5 \%$ (number-average) of the total number of particles, while it represents $1 \%$ for the copolymer with a $f$ ratio of 0.15 . Such a result was expected, and is due to the long PAA block, driving the self assembly toward smaller aggregates.

Although those polymers formed vesicles, the fraction of micelles was still largely superior. According to literature, the total copolymer length also influences the formation of polymersomes with respect to other structures. It has been shown that the formation of vesicles is easier with long chain copolymers compared to short chain copolymers. ${ }^{[127,128]}$

We have seen that $\mathrm{PMCL}_{105}-O N B-\mathrm{PAA}_{30}$ and $\mathrm{PMCL}_{30}-O N B-\mathrm{PAA}_{6}$ diblock copolymers formed polymersomes. However, in the case of $\mathrm{PMCL}_{105}-O N B-\mathrm{PAA}_{30}$, it seems that the $f$ ratio is too large, and in the case of $\mathrm{PMCL}_{30}-O N B-\mathrm{PAA}_{6}$, even though the $f$ ratio is more favorable, the total length of the copolymer may be too short. 
Therefore, based on this hypothesis, a PMCL $76^{-} \mathrm{ONB}-\mathrm{PAA}_{16}$ copolymer with intermediate $\mathrm{Mw}$, and a $f$ ratio of 0.11 was synthesized. This polymer gave the most promising results when analyzed by TEM and right angle DLS. It appears that the formation of vesicles versus micelles is favored. We thoroughly characterized this diblock using TEM, DLS, and SLS, and used it later to explore the UV-induced degradation of the vesicles.

It should be noted that micelles can be separated from vesicles via size exclusion chromatography (SEC), and that the vesicles size can be homogenized by extrusion through filters with defined pore sizes. In the case of $\mathrm{PMCL}_{76^{-}} \mathrm{ONB}-\mathrm{PAA}_{16}$ for instance, we were able to isolate polymersomes with relatively homogeneous sizes. For this copolymer, TEM micrographs reveal the presence of spherical structures of diameters ranging from 100 to $150 \mathrm{~nm}$, and only a few larger (diameter up to 500 nm, Figure 39).
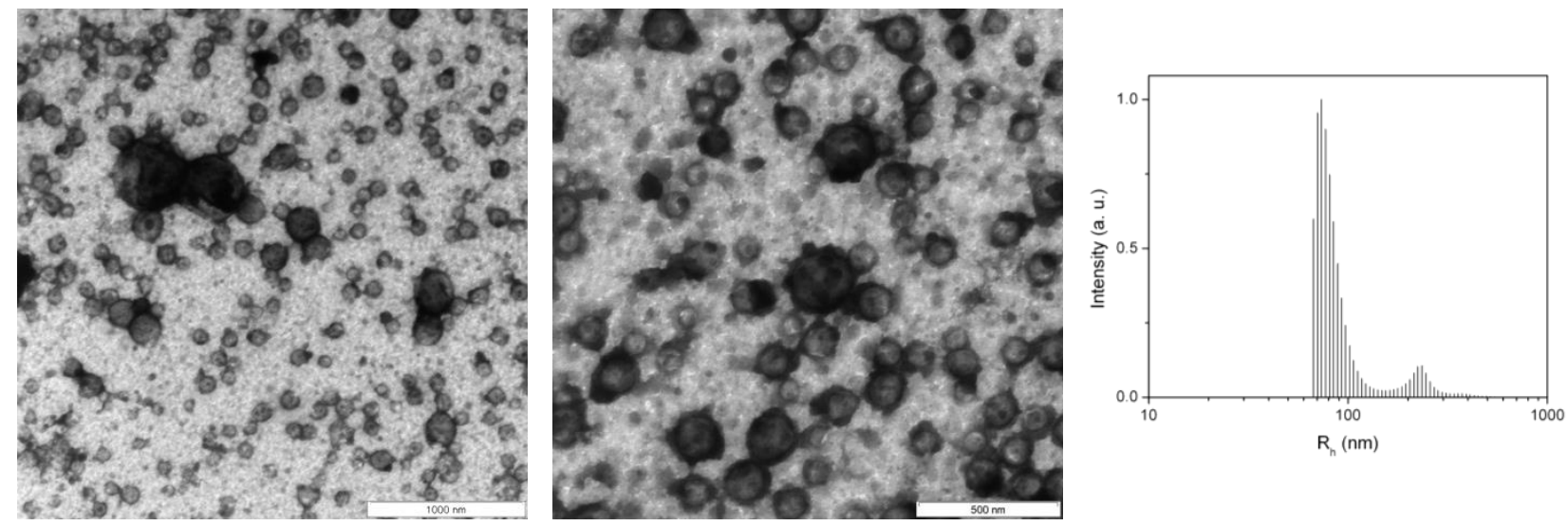

Figure 39: Size distribution (from DLS), and TEM micrographs showing vesicles formed by selfassembly of $P M C L_{76}-O N B-P A A_{16}$ in $P B S, p H$ 7.4. Divergence in size of the objects observed by TEM and DLS is attributed to the difference in size between hydrated corona chains in water (DLS), and collapsed corona chains in the dry state (TEM). TEM scale bars: 1000 and $500 \mathrm{~nm}$.

The size distribution histograms, obtained by DLS, indicate that the initial solution of self-assembled structures contains a major population with a hydrodynamic radius $\left(R_{h}\right)$ of $80 \mathrm{~nm}$. 
The major population and a secondary population with a $R_{h}$ around $200 \mathrm{~nm}$ reveal the presence of large structures that could be either vesicles or micellar aggregates (Figure 39).

Additional information on the morphology of these large structures can be gained from static light scattering. $\mathrm{PMCL}_{76}-O N B-\mathrm{PAA}_{16}$ copolymer solutions with several concentration (from 0.01 to $1 \mathrm{mg} / \mathrm{mL}$ ) were analyzed by SLS (angles from 40 to 140), to obtain the radius of gyration $\left(R_{g}\right)$. This quantity was then used to calculate the $R_{g} / R_{h}$ ratio, which is a variable used to describe the morphology of the particles in solutions. We calculated a ratio of 0.94 between the radius of gyration $\left(R_{g}\right)$ and the hydrodynamic radius $\left(R_{h}\right)$, using Cumulant analysis and Berry

plots. This value is comparable to the theoretical value given for hollow spheres $\left(R_{g} / R_{h}=1.0\right),{ }^{[129]}$ (when micelles typically have a $R_{g} / R_{h}$ ratio of 0.77 ) and represents an indication that the two populations are vesicles.

To conclude, the combination of DLS, SLS, and TEM demonstrate that, when hydrated, $\mathrm{PMCL}_{76^{-}} \mathrm{ONB}-\mathrm{PAA}_{16}$ copolymer preferentially self-assemble into vesicular structures. The next subchapter is focusing on the UV-induced modification altering the polymersome structure.

\subsection{Photodegradation of self assembled structures}

We investigate the effect of UV-irradiation on polymersome structure. We observed their changes in size and morphology, in order to understand how the system responds to the external light stimulus and to propose a plausible degradation mechanism.

\subsubsection{Experimental data}

\subsubsection{DLS}

Number-averaged size distributions of a solution of vesicles measured after different irradiation times indicate that, during the irradiation process, vesicle size decreases (Figure 40). 

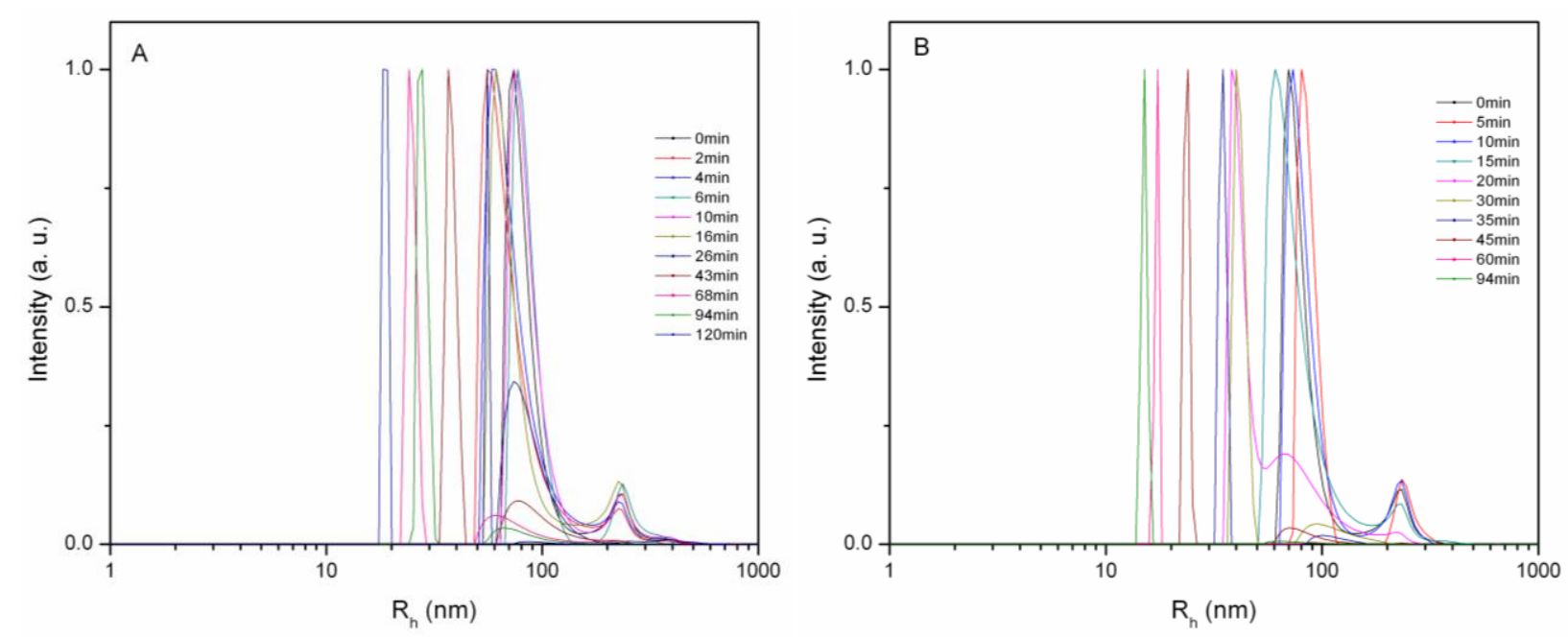

Figure 40: Number-averaged size distributions obtained by dynamic light scattering (DLS). A) $20 \mathrm{~mW}_{\mathrm{cm}} \mathrm{cm}^{-2}$; B) $200 \mathrm{~mW} . \mathrm{cm}^{-2}$. Three measurements at $90^{\circ}$ for 300 seconds each were performed on samples irradiated for different amounts of time. The correlation curves were fitted using the CONTIN method.

After 20 minutes of irradiation with a dose of $200 \mathrm{~mW} . \mathrm{cm}^{-2}$, both populations of initially observed intact vesicles (radii of 80 and $200 \mathrm{~nm}$, Figure 41, A) were significantly reduced, and a distinct population of smaller entities appeared (Figure 41, B). Conjecturably, those newly formed species, with a $R_{h}$ of around $40 \mathrm{~nm}$, are fragments of degraded vesicles. There were relatively few intact vesicles after 45 minutes of irradiation, and the major peak was associated with fragments of vesicles (Figure 41, C). In turn, these entities finally yield micellar-like structures, represented by the sharp peak with a $R_{h}<20 \mathrm{~nm}$ (Figure 41, D). Interestingly, even after 60 minutes of UV irradiation, a very small peak (accounting for 1\% of the aggregates) can still be seen at $80 \mathrm{~nm}$, indicating that a fraction of the vesicles is not destroyed or that micellar aggregates are formed (insert in Figure 41, D). 

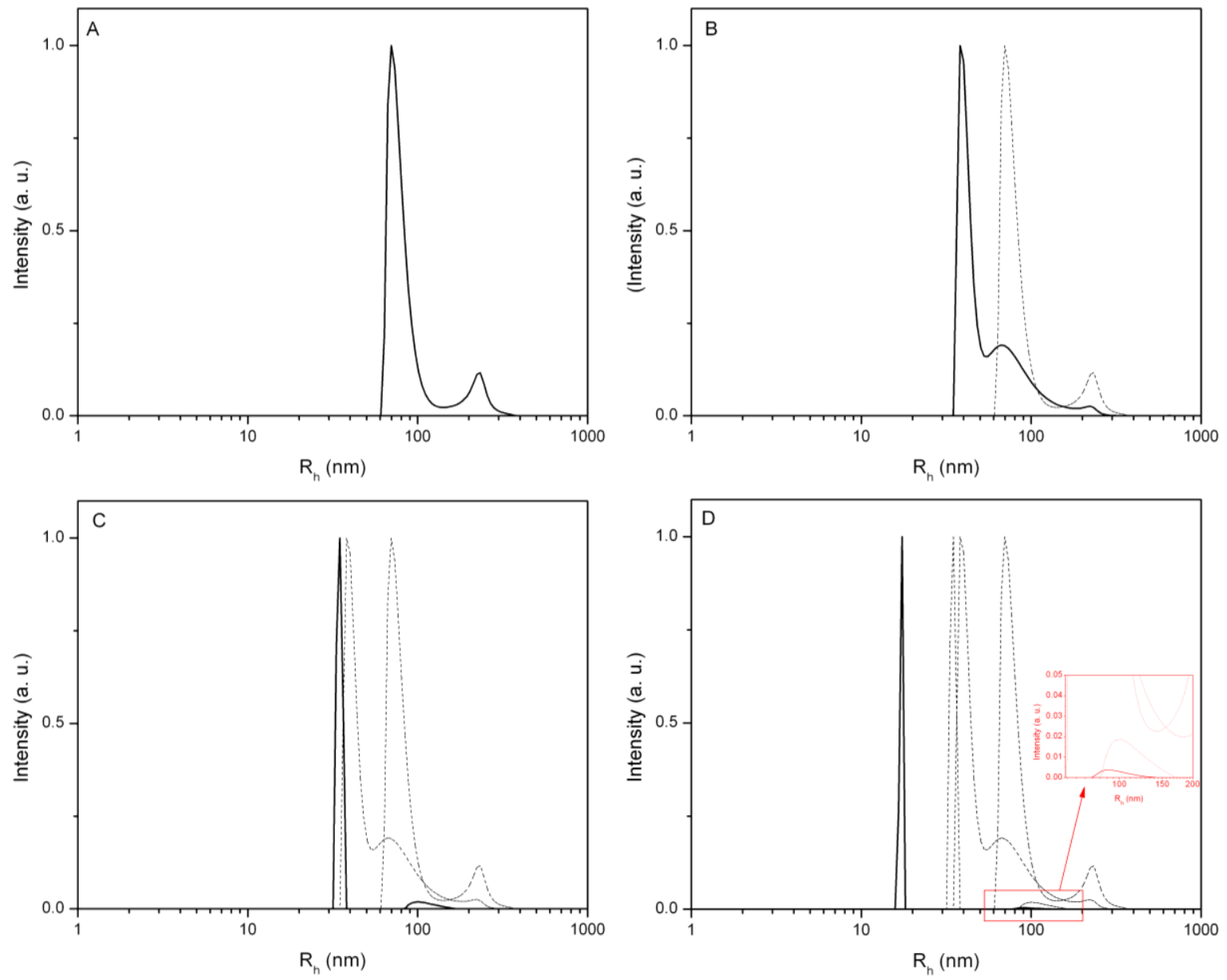

Figure 41: Number-averaged size distributions obtained by dynamic light scattering (DLS). Three measurements at $90^{\circ}$ for 300 seconds each were performed on samples irradiated (200 $\mathrm{mW} . \mathrm{cm}^{-2}$ ) for different amounts of time. The correlation curves were fitted using the CONTIN method. A) 0 minutes, B) 20 minutes $U V, C) 45$ minutes $U V$, and D) 60 minutes $U V$.

A similar degradation behavior was observed when a lower UV irradiation dose (20 $\mathrm{mW} . \mathrm{cm}^{-2}$ ) was applied to the same solution.

In addition, a significant decrease in the mean count rate during the DLS experiment was observed (Figure 42), in agreement with previously reported photodegradable micelles, ${ }^{[64]}$ and redox sensitive micelles. ${ }^{[130]}$ In general, the intensity decay is due to a lower number of aggregates in solution (correlated with the destruction of the initial particles and material precipitation), a decrease in their size, or a combination of both. Surprisingly, the UV exposure 
of the self-assembled PAA-ONB-PMCL chains is not accompanied by precipitation. This proves that, in this case, the observed decrease in mean count rate is solely associated with the disruption of vesicles to smaller particles.

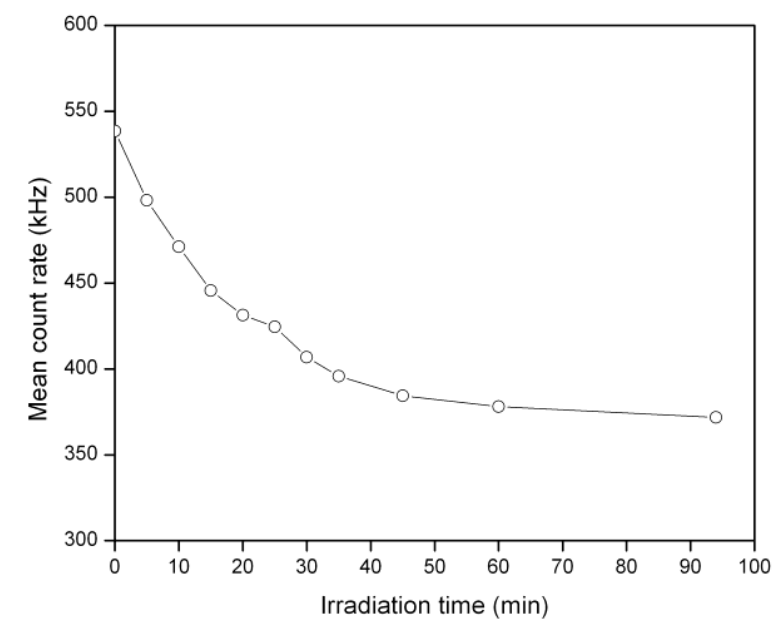

Figure 42: Plot of mean count rate vs. irradiation time $\left(200 \mathrm{~mW}^{\mathrm{cm}} \mathrm{cm}^{-2}\right)$ for DLS experiment using $A B 2$ vesicles in PBS. Values averaged over three measurements.

\subsubsection{SLS}

We performed SLS on a vesicle solution after 94 minutes of UV irradiation to gain further insight into the morphology of the degraded structures. The Berry and cumulant plots used for calculations are given in Figure 43. The $R_{g} / R_{h}$ ratio dropped from 0.94 for intact vesicles, to 0.88 , a value that is higher than the typical ratio of 0.77 , which is specific for micellar structures. The weak effect observed on the ratio indicates that we have a mixture of micelles and other bigger aggregates after UV treatment: the presence of still intact vesicles in solution, or micellar aggregates, is likely to explain this ratio. 

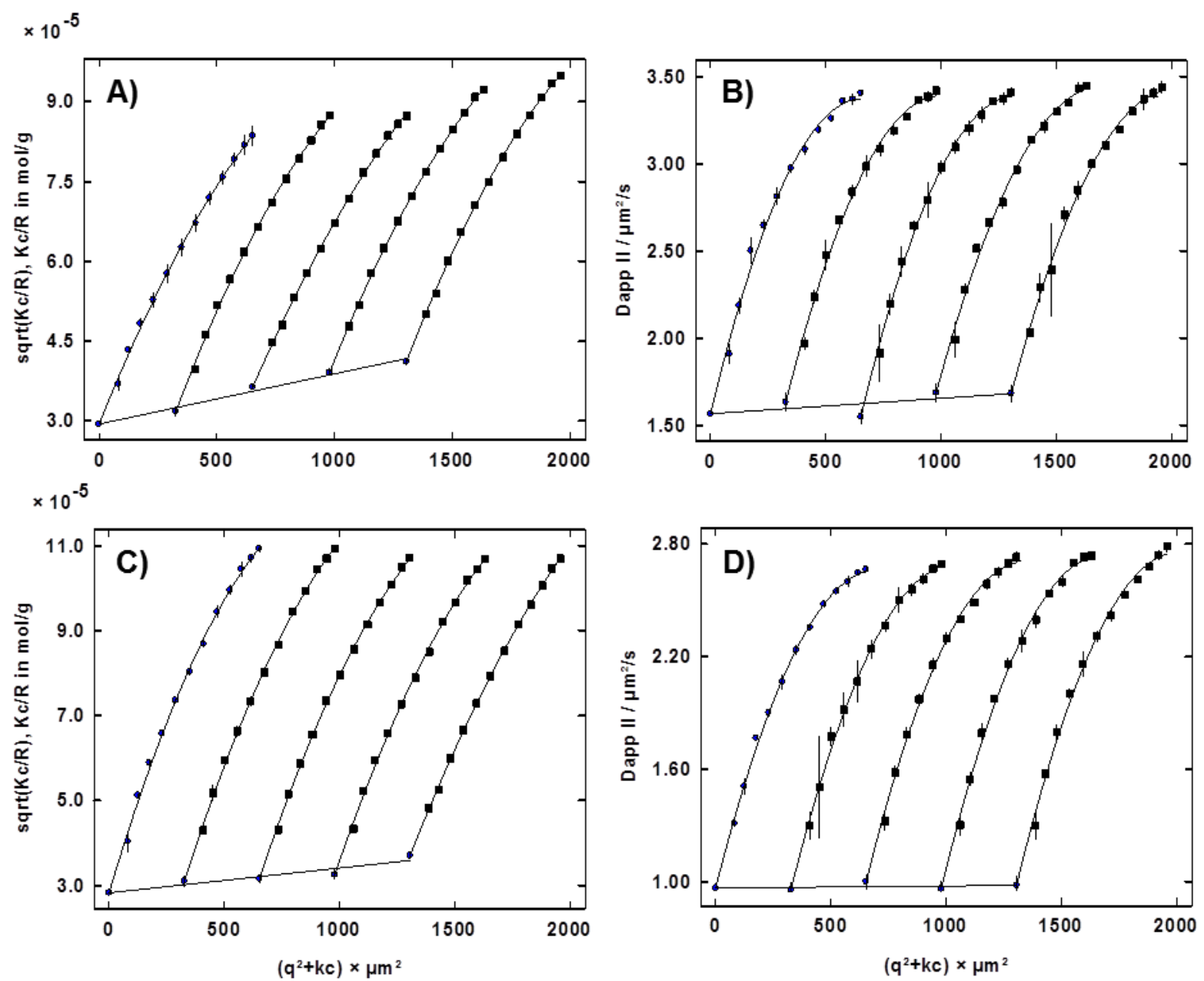

Figure 43: Berry and cumulant plots for a solution of PMCL $L_{76^{-}} \mathrm{ONB}-\mathrm{PA} A_{16}$ vesicles before and after UV exposure: A) Berry plot before $U V, B)$ cumulant analysis before $U V, C)$ Berry plot after $U V$, and D) cumulant analysis after UV. UV irradiation with $200 \mathrm{~mW} . \mathrm{cm}^{-2}$.

\subsubsection{TEM}

We applied TEM to visually observe the morphology changes upon UV irradiation. The TEM micrographs show three different morphologies, corresponding to different stages of photodegradation: vesicles, broken vesicles, and finally micellar structures (Figure 44). 


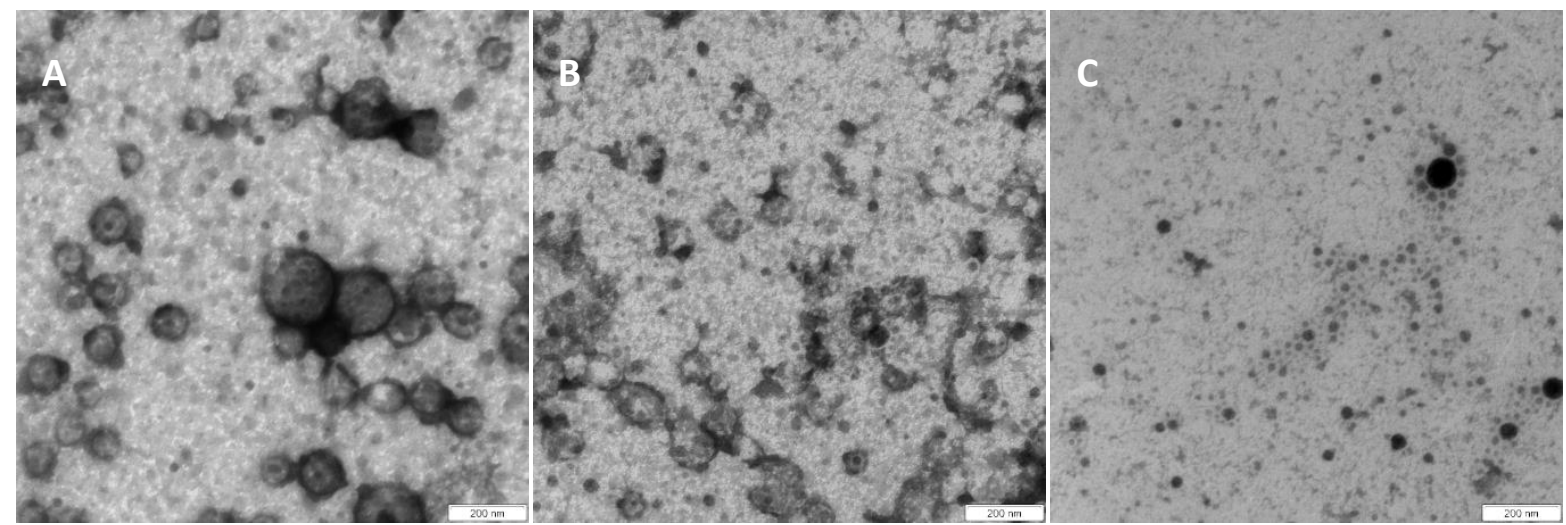

Figure 44: Morphology changes observed by TEM: (A) before UV, vesicles with diameter around 100nm, (B) 25min UV, broken vesicles and shapeless particles, and (C) 90min UV, micelles and micellar aggregates. Scale bars: $200 \mathrm{~nm}$.

This degradation sequence, based on observable morphology changes, is in good agreement with the decrease in size observed by DLS. It is also interesting to see that, according to Figure $53 \mathrm{C}$, the small fraction of big structures observed after 60 minutes of UV-irradiation (fraction with $\mathrm{Rh}=80 \mathrm{~nm}$, figure $50 \mathrm{D}$, and $\mathrm{R}_{\mathrm{g}} / \mathrm{R}_{\mathrm{h}}$ ratio of 0.88 ), results from aggregation of small micelles rather than intact vesicles. This observation supports the hypothesis of a UVinduced transition from polymersomes to micelles, which possibly aggregate.

To conclude, DLS, SLS, and TEM characterization techniques show that the UV-irradiation of PMCL-ONB-PAA polymersomes caused an unambiguous change both in size and morphology, to finally yield micellar structures.

\subsubsection{Kinetics}

The kinetics of vesicle degradation can be investigated by the change in size induced by UV irradiation over time and observed in DLS experiments (20 and $200 \mathrm{~mW} . \mathrm{cm}^{-2}$, Figure 45). When the lowest irradiation intensity was used, the decrease in size took place rather slowly. As expected, the process is faster when a higher intensity of UV is applied. This behavior correlates to the increased ONB degradation rate for higher UV intensities, ${ }^{[131]}$ and can be used to tune the release rate of encapsulated compounds. The progressive change in size and morphology is 
correlated with the destruction of the initial vesicles and with the formation of micelles, as proposed in the summary included in Figure 45.

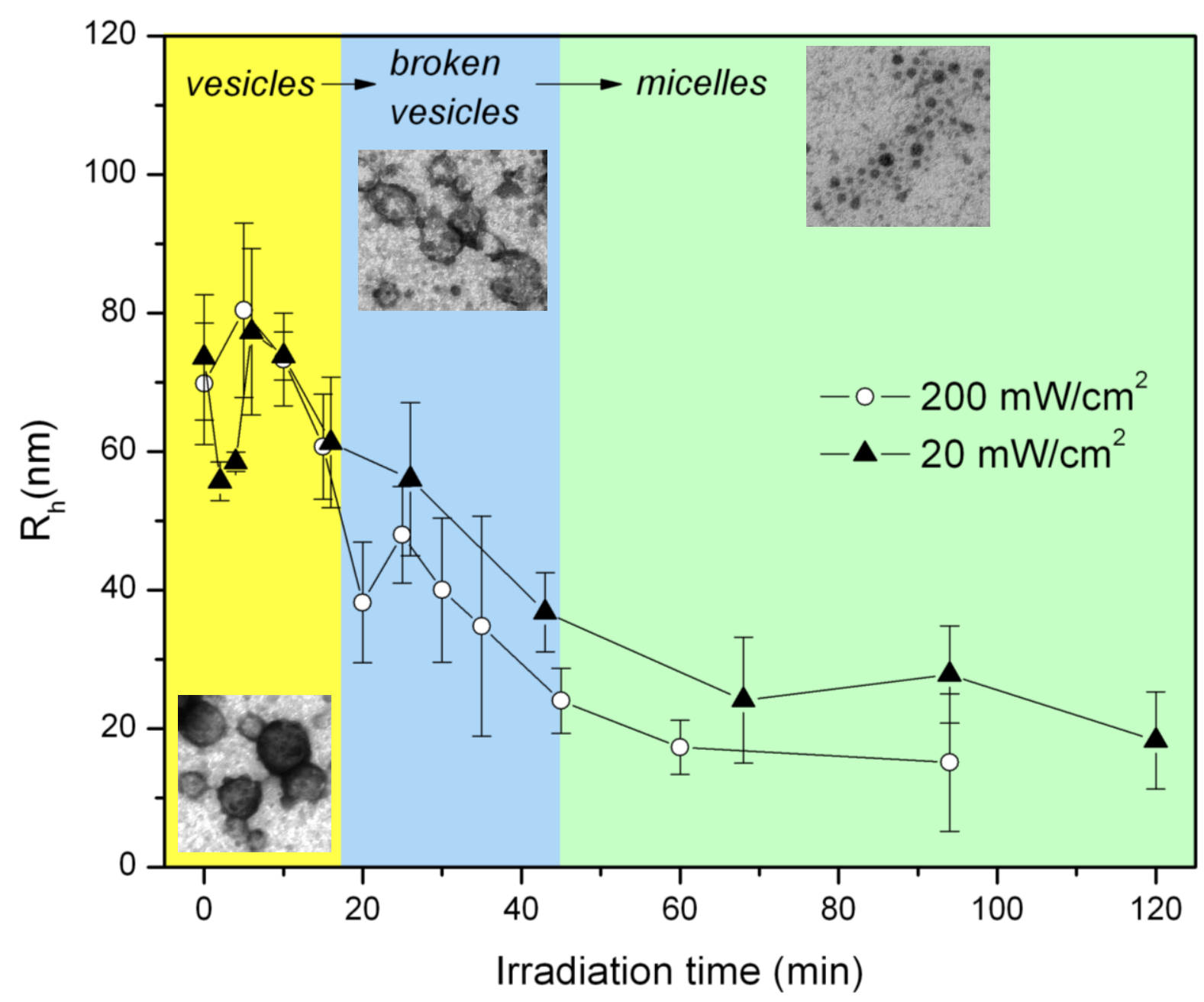

Figure 45: Evolution of $R_{h}$ vs. irradiation time, showing the transition from initially mostly vesicles, to a mixture with intermediate compounds with lower sizes, and finally to micelles.

\subsection{Explanation of mechanism}

In this section, our goal is to present a satisfactory explanation of the transition from vesicles to micelles evidenced experimentally. If a nanocarrier system is designed for in vivo 
applications, then its content must be released or made accessible, either by disruption of the vesicle, or by a permeabilisation mechanism.

\subsubsection{Common degradation mechanism for responsive polymersomes}

There are various models to describe the response of polymersomes to different stimuli, the most common being vesicle destruction and membrane permeability increase.

Although a triggered degradation mechanism of responsive polymersomes is not often discussed in detail, it is generally accepted that the destruction of vesicular structures is due to a loss of membrane integrity. ${ }^{[42]}$ In the majority of stimuli-responsive vesicles, the membrane is destabilized via the alteration or modification of one of the polymer blocks. In the most common systems, hydrophobic segments are fractionated by acidolysis. ${ }^{[8]}$ In this case, the stimulus causes a dramatic change in the hydrophilic/hydrophobic ratio $f$ (this ratio increases as the hydrophobic block is shortened), ${ }^{[132]}$ and vesicles disintegrate to form micellar structures, a transition favored by this new hydrophilic/hydrophobic balance. In other systems (triggered by $\mathrm{pH}$ or temperature), the hydrophilicity of a block can be tuned to obtain a fully hydrophilic copolymer chain, leading to dissolution of the membrane, ${ }^{[48,52,53,133]}$ and causing the vesicle's destruction.

The payload may also be released upon an increase in the permeability of the membrane, i.e. without necessarily breaking the vesicles. Such systems are actually mostly used as nanoreactors, from which a substrate is released. The permeability of the membrane may be tuned by introducing membrane proteins (such as $\mathrm{OmpF},{ }^{[134]}$ and aquaporine ${ }^{[135]}$ ), or by using polypeptides adopting helical structures in membranes. However, very few purely synthetic and triggerable polymersomes forming semi-permeable membranes have been reported. Examples generally involve the selective degradation of polymers inserted in a crosslinked membrane, thereby forming pores. ${ }^{[136,137]}$

In our approach, the degradation mechanism is not based on selective alteration of one or two blocks (whether it is degradation or hydrophilicity change), but on the physical cleavage of the diblock, at the hydrophilic/hydrophobic junction. To our knowledge, the work of Katz and coworkers, ${ }^{[79]}$ represents the only detailed example of a polymersome for which destabilization 
is induced by cleavage at the hydrophilic/hydrophobic interface. A diblock copolymer of poly(ethylene oxide) (PEO), which represents the hydrophilic part and with poly(caprolactone) (PCL) as the hydrophobic part and joined by a photocleavable moiety, self-assembled into vesicular structures. UV exposure induced a rearrangement of the cleaved PCL chains inside the membrane, resulting in a release of content. However, the vesicular structure was retained because the membrane was stabilized by the remaining, uncleaved PEO chains. After sufficient irradiation time, when all PEO chains were cleaved, the aggregates collapsed, and a precipitation occurred.

\subsubsection{Proposed mechanism for our system}

As the architecture of our diblock copolymer is similar to that of Katz, ${ }^{[79]}$ one would expect a comparable degradation mechanism. However, we observed a completely different degradation behaviour after UV irradiation: no polymer precipitation occurs, and instead micellar structures are formed. These structures, consisting of purely hydrophobic blocks, are not conventional polymeric micelles, ${ }^{3}$ but rather "shaved micelles". The existence of such polymeric superstructures, due to electrostatic stabilization in water, was reported previously. In particular, aggregates such as unimolecular micelles ${ }^{[138]}$ and dendrimers, ${ }^{[139]}$ which have a hydrophobic core, can be electrostatically stabilized in aqueous solutions by functionalization with charged carboxylic head groups. Recently, Benaglia et al. ${ }^{[140]}$ reported the formation of micelles composed of a completely hydrophobic poly(styrene)-poly(methyl acrylate) block copolymer bearing a terminal carboxylic end group; the negatively charged corona of the micelles stabilized the particles in an aqueous environment. In a similar fashion, the self assembly of linear polystyrene chains bearing a carboxylic end group into stable colloidal particles was previously reported by Liu et al. ${ }^{[141]}$

In the case of our diblock copolymer, the degradation pathway is based on the photolysis of the O-nitrobenzyl linker, generating a carboxylic end group on the cleaved poly(methyl

\footnotetext{
${ }^{3}$ I.e. micelle formed by amphiphilic diblock copolymers with a hydrophobic core and a hydrophilic corona.
} 
caprolactone) chain (Figure 8). These terminal weak acids $(\mathrm{pKa} \sim 4.5)$ are negatively charged under the conditions that we use (i.e. PBS buffer, $\mathrm{pH}=7.4$ ).

Hence, directly after irradiation, there is a strong electrostatic repulsion in the outer part of the membrane (polymer-water interface) due to the ionic groups generated by cleavage of the ONB linker. A rearrangement takes place in the membrane in order to minimize this repulsion, due to an increase of the surface area available for negative charges. Because the area available for ionic head groups is proportional to $1 / R$ (where $R$ is the radius of the spherical particle), ${ }^{[141]}$ the morphology will evolve from almost flat bilayers (extremely low curvature, high $R$ value) to more favorable micellar structures (small $R$, high curvature).

Therefore, we propose that the supramolecular structures resulting from UV irradiation of PMCL-ONB-PAA vesicles are micelle-like particles formed by aggregated PMCL chains, stabilized by an ionic corona of charged carboxylic end groups (see Figure 46). Additionally, uncleaved PMCL-ONB-PAA chains contribute to the stabilization of these aggregates in the early stage of the degradation process, explaining the transition structures observed by TEM and DLS. The size of the structures (approx. $20 \mathrm{~nm}$ in hydrodynamic radius) clearly indicates that they are formed by several PMCL chains. A putative scheme of the degradation mechanism is summarized in Figure 46. 

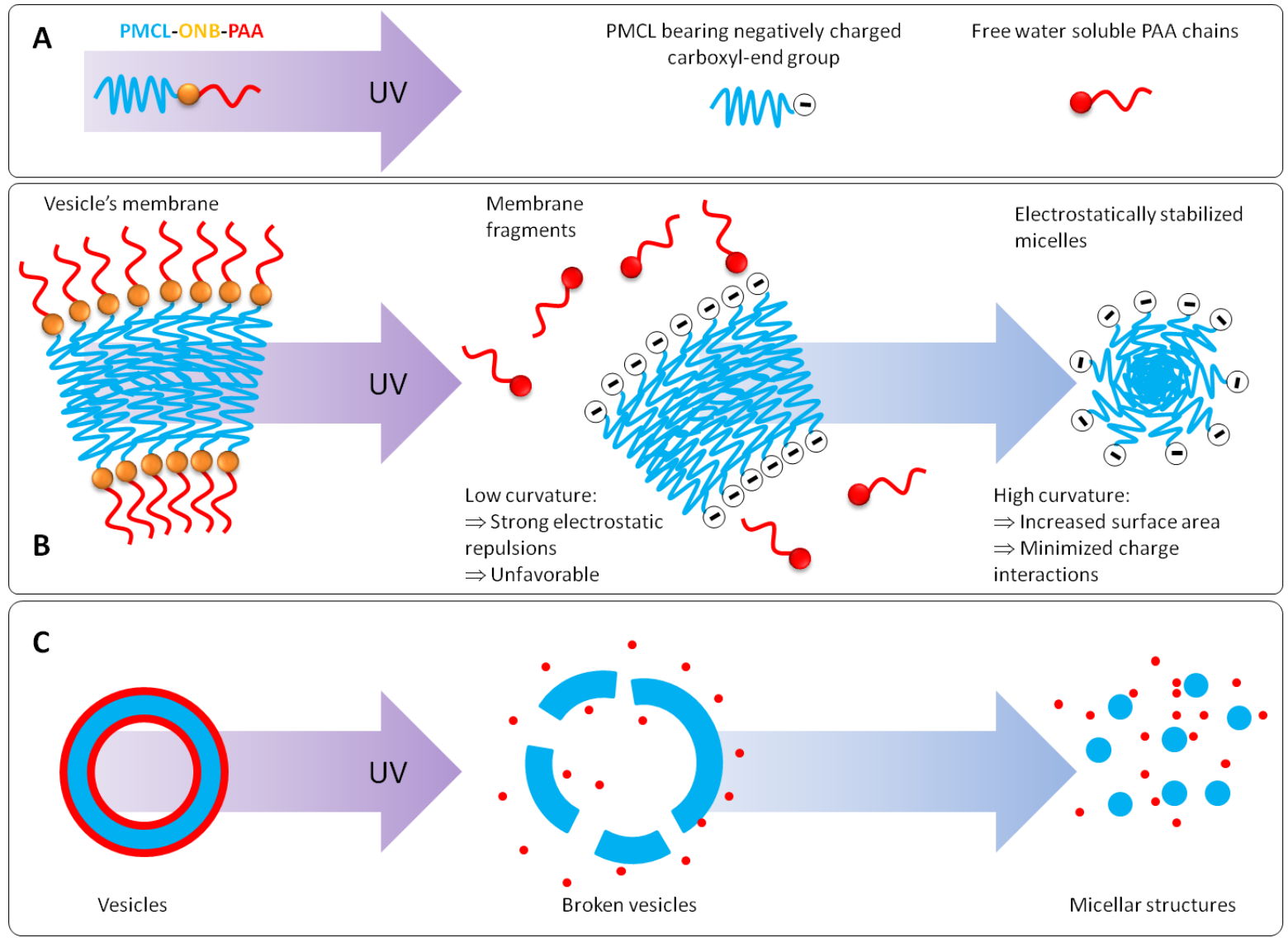

Figure 46: Graphical summary depicting the proposed mechanism of polymersome degradation. A) molecular level: the diblock copolymer is rapidly cleaved upon UV irradiation; B) supramolecular level: as a result of chain scission, packing of the PMCL chains forming the membrane is progressively destabilized and evolves into a more favorable arrangement; $C$ ) aggregate morphology: from vesicles to broken vesicles to stabilized micellar structures.

The different degradation behaviors of our diblock copolymer compared to that reported by Katz et $a l .{ }^{[43]}$ is due to the chemical nature of the photosensitive linker: O-nitrobenzyl linker yields a $\mathrm{COOH}$ residue upon cleavage, whereas the $\mathrm{N}$-nitrobenzyl linker yields an amide residue. ${ }^{[142]}$ As the amide is uncharged at physiological $\mathrm{pH}$, there is no driving force toward the formation of micellar structures. In addition, poly(caprolactone), as a hydrophobic block, ${ }^{[43]}$ is a crystalline polymer with reduced chain mobility, limiting a complete reorganization of the hydrophobic segments after cleavage. ${ }^{[3]}$ In the case of PMCL, a totally amorphous homopolymer, ${ }^{[11]}$ the flexibility of the chains is such that it provides fluidity to the membrane, 
which is able to adopt an alternate conformation and evolve toward a newly formed supramolecular structure. 


\section{Encapsulation and release of hydrophilic molecules}

\subsection{Encapsulation of low molecular weight molecules}

\subsubsection{Fluorescein release}

In order to test whether this system can be used as a phototriggered nanocarrier, we studied the encapsulation of small hydrophilic molecules inside the aqueous cavity, and their release upon UV irradiation.

To make sure that fluorescein is a suitable dye resistant to the conditions we use, measurements were carried out on a $1 \mu \mathrm{M}$ fluorescein solution in PBS, with the harshest irradiation conditions employed $\left(700 \mathrm{~mW} / \mathrm{cm}^{2}\right)$. Even if fluorescein is subjected to photobleaching over a period of 60 minutes of irradiation, the decrease in fluorescence is minimal, and should not interfere with qualitative experiments.

We also studied the fluorescence emission of the empty vesicles, to study their potential fluorescence due to the presence of the photoactive ONB linker in the polymer chains. The fluorescence of the polymer self-assemblies without any cargo was first studied, using the excitation wavelength of Fluorescein $(498 \mathrm{~nm})$. We observed that the fluorescence emission intensity is actually decreasing when UV light is applied.

Due to the relative difficulty to find molecules suitable for our encapsulation experiments (i.e. easily detected and inert toward UV irradiation), and assuming that the combined effects of fluorescein photobleaching and fluorescence decrease from the polymer itself were not dramatic, we pursued the preliminary release experiments with this dye.

The loading of polymersomes with fluorescein and its release as a result of the exposure of polymersomes to UV was followed by fluorescence spectroscopy at different UV energies (Figure 47). A significant increase in fluorescence intensity over time was obtained for the solutions of loaded vesicles exposed to UV, which was associated with the release of fluorescein (Figure 47, A - C). The solution of loaded vesicles that was kept in the dark showed no 
fluorescence increase over time, indicating that there was no leakage without UV irradiation (Figure 47, D).
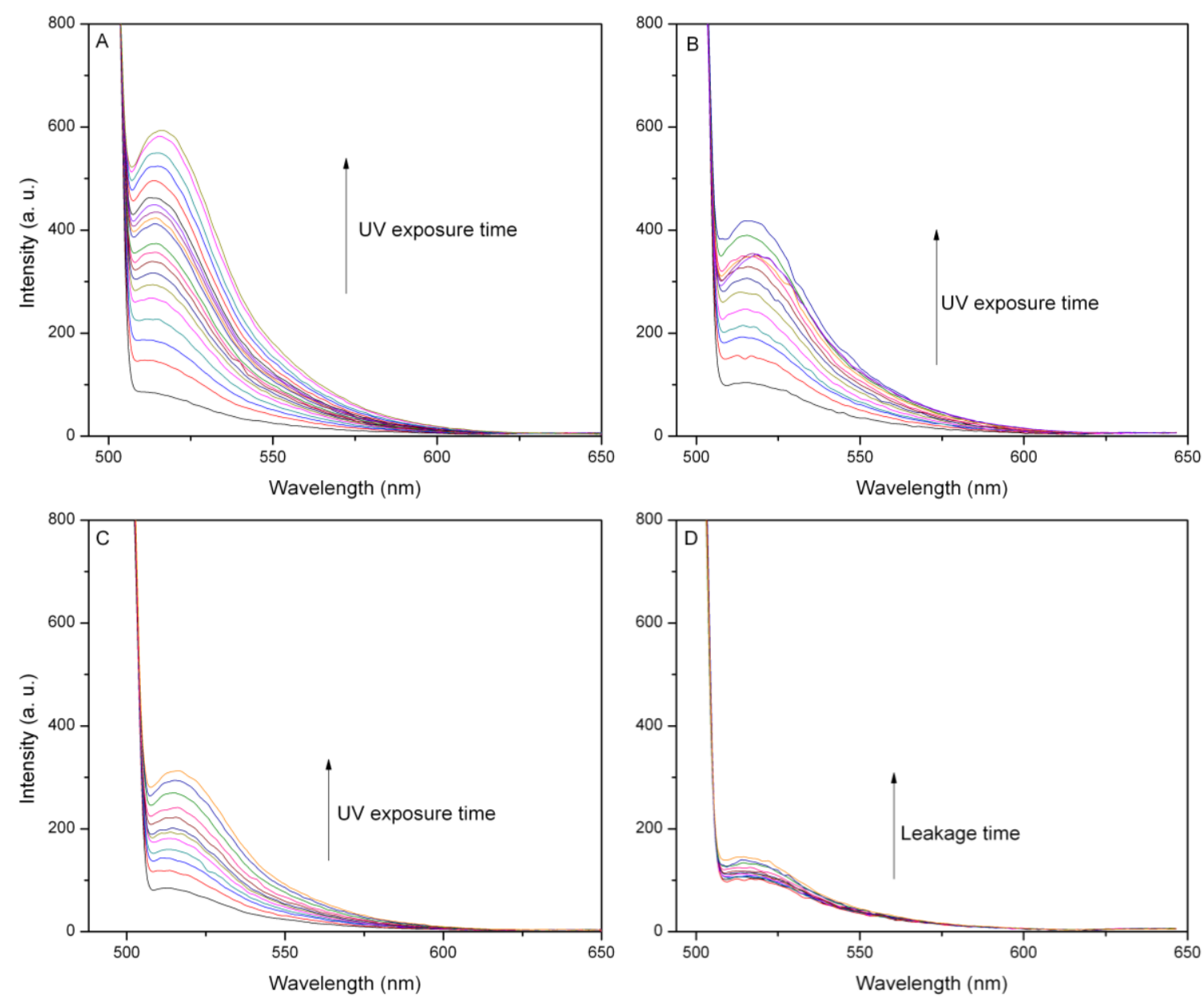

Figure 47: Fluorescence spectra of fluorescein-loaded polymersomes exposed to different UV doses, showing the increase in fluorescence over time (from 0 to 311 minutes) due to release of the dye from a state of high concentration inside vesicles (self-quenching regime) to free dye in water (unquenched regime). A) Irradiation with $700 \mathrm{mW.cm}$; B) irradiation with $200 \mathrm{~mW} . \mathrm{cm}^{-2}$;

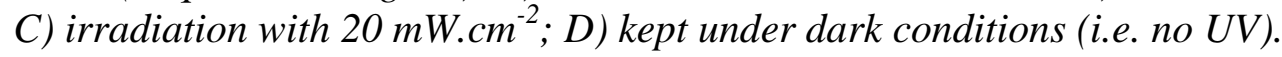

In addition, release rates depended on the UV intensity: by increasing the UV intensity, a more rapid release was obtained (Figure 47 and Figure 48, A). While 200 minutes were necessary to release about $40 \%$ of the encapsulated fluorescein by irradiation with an intensity of 
$20 \mathrm{~mW} . \mathrm{cm}^{-2}$, only 20 minutes were necessary to release a similar fraction when an intensity of $700 \mathrm{~mW} . \mathrm{cm}^{-2}$ was used (Figure 48, B). The fact that the UV triggered release from PMCL-ONBPAA vesicles is highly dependent on the intensity of the irradiation can serve to control the release process.
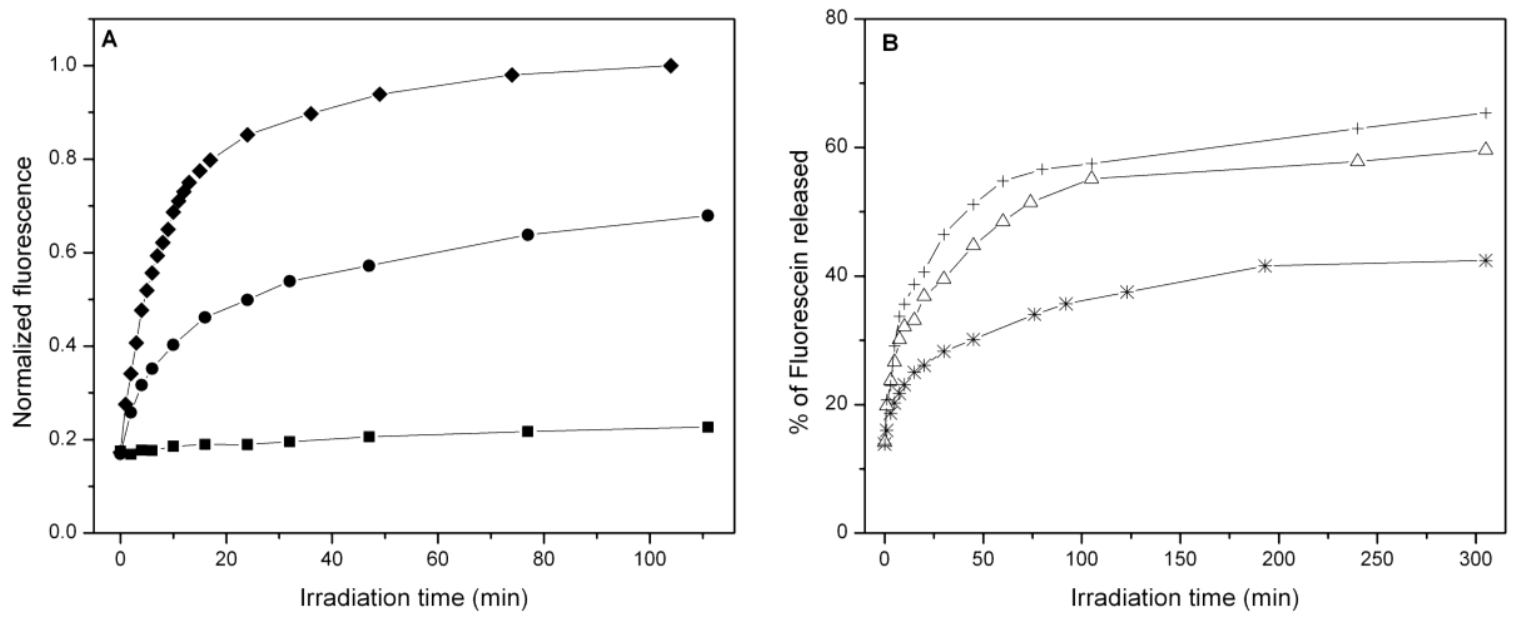

Figure 48: (A) plot of normalized fluorescence (maximum emission intensity at $\lambda=513 \mathrm{~nm}$ ) vs. irradiation time for fluorescein-loaded polymersomes: ( $\boldsymbol{( \boldsymbol { C } )}$ no irradiation, ( $\boldsymbol{O}$ ) irradiation with $20 \mathrm{~mW} . \mathrm{cm}^{-2}$, and ( irradiation with $700 \mathrm{~mW}^{-\mathrm{cm}^{-2}}$. (B) percentage of Fluorescein released vs. irradiation time for fluorescein-loaded polymersomes exposed to different $U V$ energies, showing energy dependent release kinetics: (*) $20 \mathrm{~mW} . \mathrm{cm}^{-2}$, ( $\triangle$ ) $200 \mathrm{~mW} . \mathrm{cm}^{-2}$, and (+) $700 \mathrm{~mW} . \mathrm{cm}^{-2}$.

\subsubsection{Release of ATTO655 followed by fluorescence correlation spectroscopy (FCS)}

We used FCS to gain further insight into release behavior from PMCL-ONB-PAA polymersomes because it is a very sensitive method, up to single molecule detection. In FCS, the fluctuations in the emission signal in time are decrypted using autocorrelation functions. When a persistent pattern caused by different physical parameters is found, essential information on

\footnotetext{
${ }^{4}$ The percentage of dye released was estimated from comparison with the maximum fluorescence emission intensity obtained after mechanical destruction of the vesicles kept under dark conditions. Due to measurement approximations (fluorescence signal affected by the background signal from polymer, and possibly by photobleaching of the dye), the percentage of fluorescein released should not be taken at face value.
} 
molecular dynamics, such as diffusion properties, can be derived from the autocorrelation curves. FCS provides diffusion time, number, and brightness of freely diffusing fluorescent molecules through a confocal volume of around $1 \mathrm{fL}$. The diffusion time can be used to determine the hydrodynamic radius of the particles by using the Stokes-Einstein equation. ${ }^{[62]} \mathrm{We}$ used FCS both to determine the amount of released dye from polymersomes as a function of irradiation time, and to calculate the size of the polymer superstructures present in solution. For encapsulation, we used ATTO655 dye instead of fluorescein, because it is more photostable while having a similar molecular weight (Figure 49).
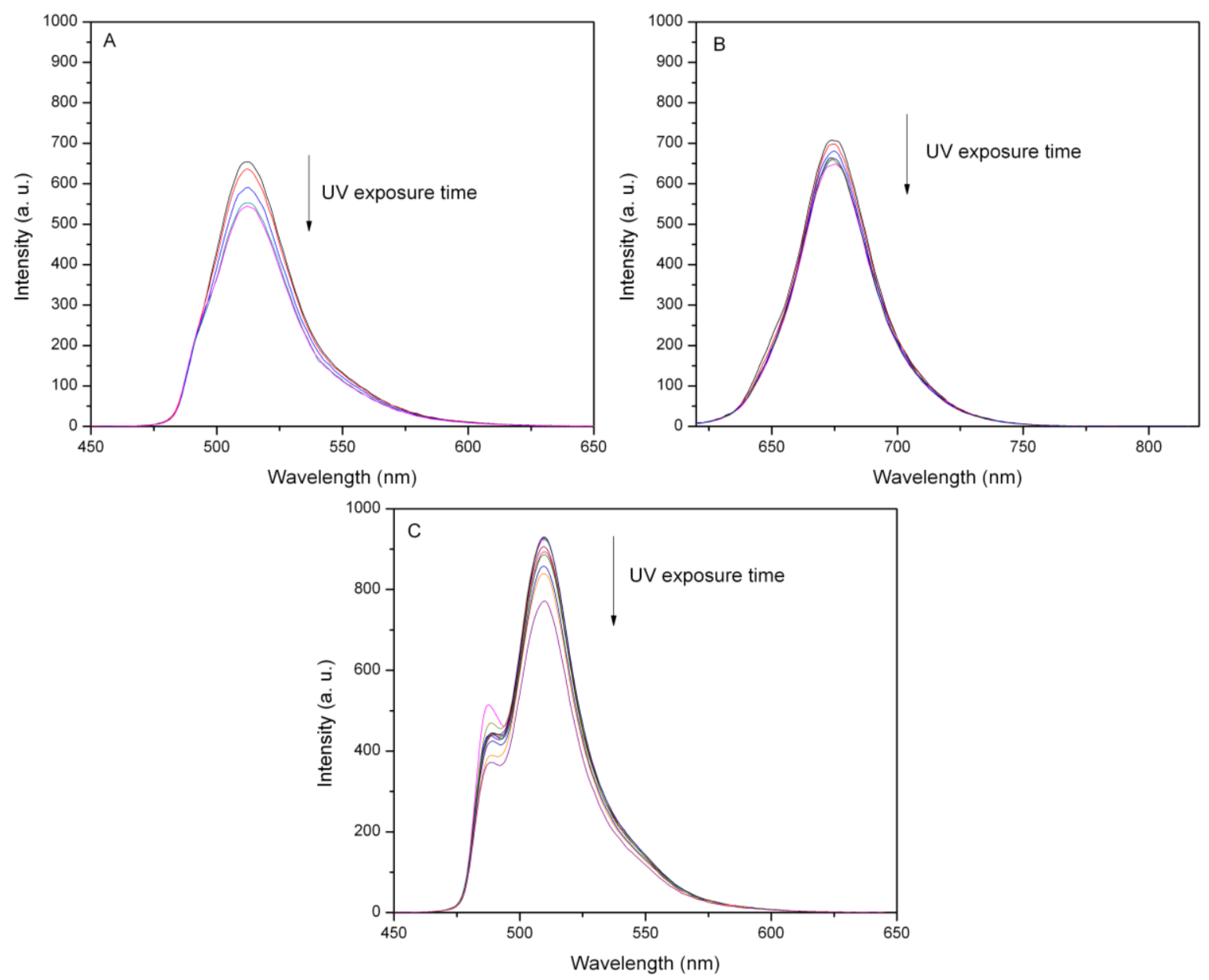

Figure 49: Photobleaching of encapsulated species: A) fluorescein, B) ATTO655, and C) eGFP. All experiments were performed for 0 to 100 minutes of irradiation $\left(200 \mathrm{~mW}^{\mathrm{cm}} \mathrm{cm}^{-2}\right.$. 


\section{Encapsulation and release of hydrophilic molecules}

We analyzed the autocorrelation curves using the least-squares fitting for two populations: free dye and polymersomes (Figure 50). ${ }^{[143]}$ The average diffusion time $\left(\tau_{D}\right)$ of the free dye was $44 \mu \mathrm{s}$, while the $\tau_{D}$ for encapsulated dye inside polymersomes was $15.64 \mathrm{~ms}$. Nearly $70 \%$ of dye was released after 15 minutes of UV irradiation, (Table 5).

Table 5: FCS results for vesicles encapsulating ATTO655 dye.

\begin{tabular}{|c|c|c|c|c|c|c|}
\hline Sample & $\mathrm{F}_{1}[\%]^{\mathrm{a}}$ & $\tau_{\mathrm{D}, 1}[\mu \mathrm{s}]^{\mathrm{b}}$ & $\mathrm{R}_{\mathrm{h}, 1}[\mathrm{~nm}]^{\mathrm{c}}$ & $\mathrm{F}_{2}[\%]^{\mathrm{a}}$ & $\tau_{\mathrm{D}, 2}[\mu \mathrm{s}]^{\mathrm{b}}$ & $\mathrm{R}_{\mathrm{h}, 2}[\mathrm{~nm}]^{\mathrm{c}}$ \\
\hline ATTO655, 52nM in PBS & 100 & 44 & 0.5 & NA [d] & NA [d] & NA [d] \\
\hline Loaded vesicles, $\mathrm{UV}=0 \mathrm{~min}$ & 0.01 & 44 & 0.5 & 99.9 & 15645 & 171.9 \\
\hline Loaded vesicles, $\mathrm{UV}=15 \mathrm{~min}$ & 70.1 & 44 & 0.5 & 29.9 & 5015 & 55.1 \\
\hline Loaded vesicles, $\mathrm{UV}=25 \mathrm{~min}$ & 96.4 & 44 & 0.5 & 3.6 & 1486 & 16.3 \\
\hline Loaded vesicles, $\mathrm{UV}=45 \mathrm{~min}$ & 97.9 & 44 & 0.5 & 2.1 & 950 & 10.4 \\
\hline
\end{tabular}

The fraction of free dye in solution increased with longer UV irradiation, and after 45 minutes ATTO655 was completely released (Figure 50). 

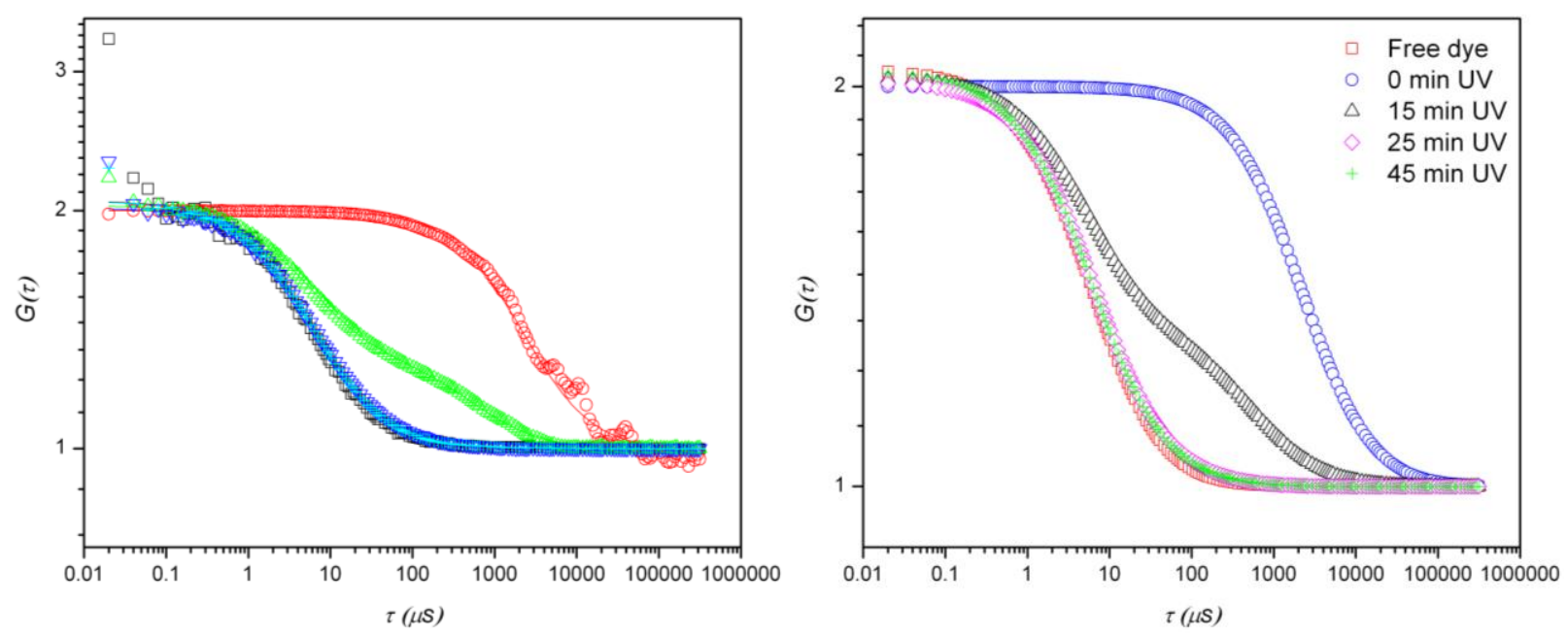

Figure 50: Left: experimental FCS autocorrelation curves (symbols) and fitted curves (plain lines) for ATTO655 release experiment. Right: FCS autocorrelation curves obtained for a solution of free ATTO655 in PBS ( $\square$ ), a solution of polymersomes loaded with ATTO655 under dark conditions ( $O)$, the same solution exposed to $U V(15$ minutes) $(\triangle)$, to 25 minutes $U V(\diamond)$, and to 45 minutes $U V(+)$. All curves were normalized to 2. UV energy: $200 \mathrm{~mW} . \mathrm{cm}^{-2}$.

The diffusion times were used to estimate the $R_{h}$ of loaded polymersomes and degraded supramolecular structures, respectively (see Table 5). Upon UV irradiation, the hydrodynamic radius of the supramolecular structures progressively decreased from 172 to $10 \mathrm{~nm}$, in agreement with dynamic light scattering. Thus, UV irradiation triggers the disassembly of the polymersomes $\left(R_{h}\right.$ of $\left.172 \mathrm{~nm}\right)$ to yield micellar structures $\left(R_{h}\right.$ of $\left.10 \mathrm{~nm}\right)$.

\subsection{Encapsulation of protein enhanced green fluorescent protein (eGFP)}

To show the versatility of our system in respect with large molecules such as DNA and proteins, we investigated the encapsulation and release of enhanced green fluorescent protein with FCS.

The diffusion time for eGFP is $98.5 \mu \mathrm{s}$, while it is $10.4 \mathrm{~ms}$ for the encapsulated proteins inside polymersomes (Table 6). The diffusion time of free proteins corresponds to a hydrodynamic radius of $2.2 \mathrm{~nm}$, in good agreement with the reported radius of $2.3 \mathrm{~nm} .^{[144]}$ 
Table 6: FCS evaluation of polymersomes loaded with eGFP.

\begin{tabular}{lrrrrrr}
\hline Sample & $\mathrm{F}_{1}[\%]^{\mathrm{a}}$ & $\tau_{\mathrm{D}, 1}[\mu \mathrm{s}]^{\mathrm{b}}$ & $\mathrm{R}_{\mathrm{h}, 1}[\mathrm{~nm}]^{\mathrm{c}}$ & $\mathrm{F}_{2}[\%]^{\mathrm{a}}$ & $\tau_{\mathrm{D}, 2}[\mu \mathrm{s}]^{\mathrm{b}}$ & $\mathrm{R}_{\mathrm{h}, 2}[\mathrm{~nm}]^{\mathrm{c}}$ \\
\hline eGFP, 60nM in PBS & 100 & 98.5 & 2.2 & $\mathrm{NA}[\mathrm{d}]$ & $\mathrm{NA}[\mathrm{d}]$ & $\mathrm{NA}[\mathrm{d}]$ \\
Loaded vesicles, UV = 0 min & 0 & 98.5 & 2.2 & 100 & 10384 & 231 \\
Loaded vesicles, UV = 5 min & 61 & 98.5 & 2.2 & 39 & 10096 & 224 \\
Loaded vesicles, UV = $10 \mathrm{~min}$ & 69 & 98.5 & 2.2 & 31 & 5691 & 126 \\
Loaded vesicles, UV = 23 min & 79 & 98.5 & 2.2 & 21 & 2439 & 54 \\
Loaded vesicles, UV = 43 min & 77 & 98.5 & 2.2 & 23 & 550 & 12 \\
Loaded vesicles, UV = 60 min & 83 & 98.5 & 2.2 & 17 & 694 & 15 \\
\hline${ }^{a}$ Fraction of component n. ${ }^{\mathrm{b}}$ Average diffusion time of fraction n. ${ }^{\mathrm{c}}$ Hydrodynamic radius of fraction n. ${ }^{\mathrm{d}}$ Not \\
applicable.
\end{tabular}

The solution of encapsulated eGFP kept in dark conditions did not present free protein (Table 6). After UV irradiation, the autocorrelation curve can be fitted by taking into account the presence of two populations, labeled $F_{1}$ and $F_{2}$, respectively free and encapsulated protein (Figure 51). As the fraction of free eGFP in solution ( $\mathrm{F}_{1}$, associated with $\left.\tau_{D}=98.5 \mu \mathrm{s}\right)$ is rapidly increasing from 0 to $80 \%$ with UV exposure time, it indicates that the protein is gradually release in response to the UV trigger. 

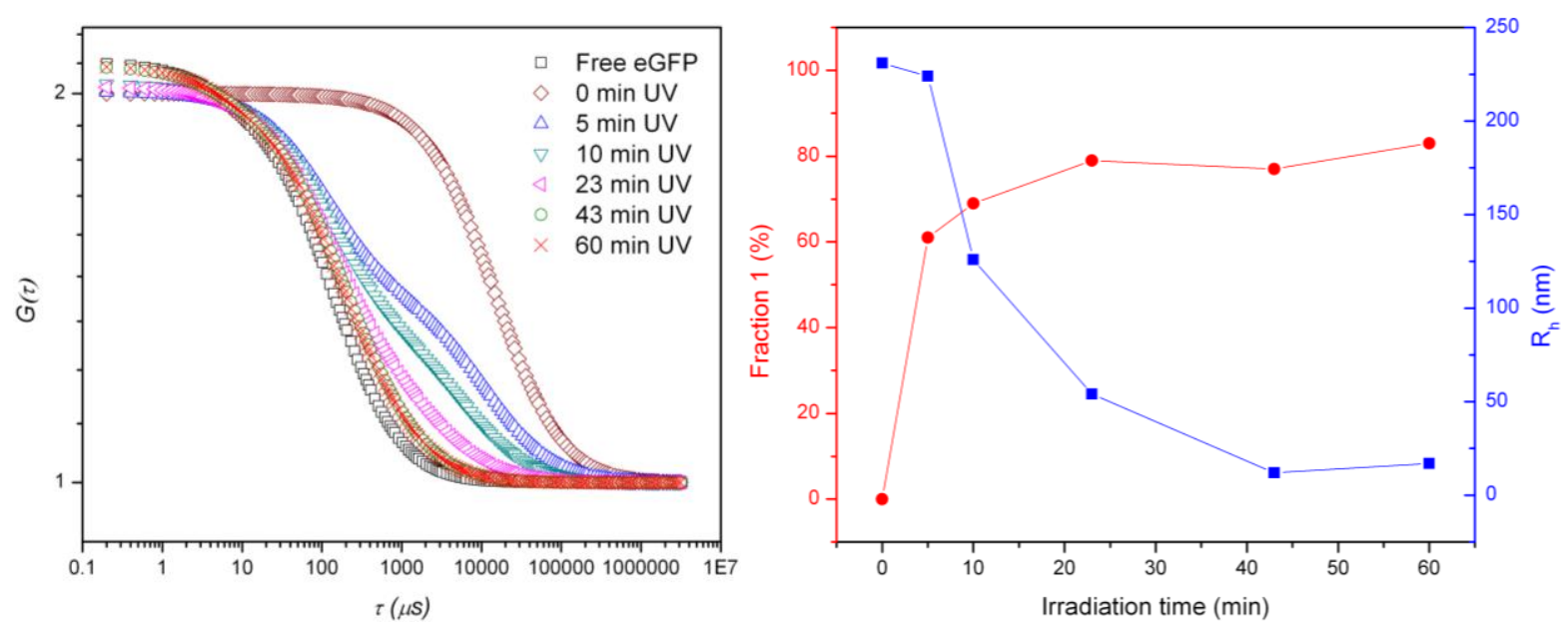

Figure 51: Left: fitted FCS correlation curves of free eGFP and polymersomes loaded with eGFP irradiated with increasing amount of time. Right: percentage of eGFP free in solution (fraction 1), and hydrodynamic radius $\left(R_{h}\right)$ of supramolecular structures plotted as a function of irradiation time. All curves were normalized to 2. UV energy: $200 \mathrm{~mW} . \mathrm{cm}^{-2}$.

In addition, the size of self-assembled structures dramatically changed upon UV irradiation. The fraction of eGFP released in solution correlates to the size decrease of the structures (Figure 51). The change of the hydrodynamic radius upon UV exposure is in good agreement with the values obtained by dynamic light scattering, and support the process of UVmediated transition from vesicles to micelles, with a simultaneous release of the protein.

The release profiles of different payloads, ranging from small molecular weight compounds up to proteins, correlate with the time scale associated with the disassembly process of the vesicles. They indicate an immediate and graded response (as a function of UV intensity) of the polymersomes to the stimulus (UV light). When the light is switched on, both the transition from the vesicle to micelle and the release start simultaneously. It shows that our system is sensitive enough to respond to the UV stimulus even if there is still a fraction of polymer chains uncleaved. Vesicles' membranes are destabilized and are rendered permeable for the encapsulated molecules in the very first minutes of irradiation. 


\section{Conclusions \& Outlook}

The goal of this thesis was to design smart polymeric nanocarriers for the encapsulation and triggered release of hydrophilic molecules. Apart from a few examples of micelles and micrometer-size vesicles, the lack of photoresponsive vesicles with ideal characteristics to be used as DDS motivated this work. In this thesis we proposed a simple bottom-up approach to fabricate polymeric vesicles obtained by the self assembly of light-sensitive building blocks (amphiphilic photocleavable block copolymers), which are able to encapsulate hydrophilic compounds, and to release them in a controlled manner.

We started with the synthesis of a new photodegradable linker, ONB, designed to initiate the independent growth of two polymer chains, using living polymerization techniques, ROP and ATRP. This synthesis route allowed us to prepare $A B$ block copolymers comprising a photocleavable moiety connecting A to $\mathrm{B}$, with well defined molecular weights and narrow molecular weight distributions. We worked with biocompatible and biodegradable PMCL (A) and PAA (B), but the versatility of our synthesis route allows various other combinations for different applications. Actually in our case, the technique allows a convenient preparation of "multi"-responsive block copolymers and superstructures with the use of $\mathrm{pH}$-sensitive hydrophilic block (PAA).

Upon UV exposure, with energies as low as $20 \mathrm{~mW} / \mathrm{cm}^{-2}$, PMCL-ONB-PAA block copolymers were effectively cleaved into PMCL and PAA homopolymers after light-induced degradation of the ONB linker. The degradation was shown to be both rapid and quantitative.

In buffer solution at physiological $\mathrm{pH}$, the amphiphilic block copolymers were shown to self-assemble into polymeric vesicles, with diameters below 200nm, and stable over several days indicating kinetically trapped structures. The vesicles undergo dramatic structural changes after a few minutes of exposition to UV-irradiation. A clear transition from vesicular structures to "shaved" micelles was evidenced, on the basis of size and morphology changes, and according to an original degradation mechanism. 
We exploited the complete degradation of the vesicles upon UV-trigger to show that they can be used as efficient DDS. We demonstrated the ability of the vesicles to encapsulate payloads ranging from small molecular weight hydrophilic molecules (Fluorescein and ATTO655) up to proteins (eGFP), supporting the versatility of our system in various therapeutic applications. The payloads were released within minutes of UV-light irradiation. In addition, the release rates were shown to be dependent on UV intensity, indicating that the inner content of the photodegradable polymersomes can be released in a controlled manner, supporting the system as an efficient stimuli-responsive drug delivery system.

Several modifications could be envisaged to further improve or tune our system. Concerning drug delivery, one possibility to adjust the release kinetics could be to blend an inert copolymer with the photocleavable copolymer to form the membrane. It has been shown that for blends of inert and hydrolysable copolymers forming vesicles, rates of release are linearly dependent on the amount of degradable copolymer blended in the membrane, in turn providing tunable release to the system. ${ }^{[145]}$ In a similar fashion, we expect that by mixing a PMCL-ONBPAA copolymer with a PMCL-PAA copolymer inert toward UV irradiation, the degradation of the vesicles would be slower. The release rates could even be correlated to the number of photocleavable moieties, since PMCL-ONB-PAA copolymers comprise a single linker per chain.

Another way to control the release rates would be to strengthen the corona of the polymersomes, by crosslinking the corona forming chains. ${ }^{[48,146]}$ Such a technique is easily applicable to PAA chains, either by amidation with diamines, ${ }^{[36,116]}$ or via thermally induced anhydride formation. ${ }^{[147]}$ As an example, stiff capsules were produced by hydrolyzing the polyester core of PCL-PAA micelles with crosslinked PAA, and the remaining crosslinked PAA shell was shown to be permeable to small molecules. ${ }^{[36]}$ In our system, by controlling the amount of PAA crosslinking, we could tune the extent of membrane degradation, hence retarding the vesicle disruption, providing control over release rate.

Finally, the degradation of the ONB linker induced by light with other wavelength was not investigated in this thesis. In particular, since ONB is sensitive to 2-photon IR, it would be of 
90 | Conclusions

high interest for biomedical applications to study the encapsulation and release of compounds with near-IR irradiation (700 to $1000 \mathrm{~nm}$ ). 


\section{Experimental part}

\subsection{Materials}

Synthesis: All commercially available solvents and reagents were used as received unless specified otherwise. 5-Hydroxy-2-nitrobenzyl alcohol (Aldrich, 97\%), a-bromoisobutyryl bromide $\left(\mathrm{BiBB}\right.$, Aldrich, 97\%), triethylamine $\left(\mathrm{Et}_{3} \mathrm{~N}\right)$, copper bromide $(\mathrm{CuBr})$, tin(II) 2ethylhexanoate $\quad\left[\mathrm{Sn}(\mathrm{Oct})_{2}\right], \quad$ trifluoroacetic acid (TFA), $\mathrm{N}, \mathrm{N}, \mathrm{N}$, $, N^{\prime}, \mathrm{N}^{\prime}$ ', pentamethyldiethylenetriamine (PMDETA), dichloromethane (DCM), tert-butyl acrylate (tBA), ethyl acetate (EtOAc), and Dulbecco's phosphate-buffered saline (PBS) were purchased from Aldrich. $\gamma$-methyl-e-caprolactone was synthesized according to a protocol described elsewhere.[23] $\mathrm{Sn}(\mathrm{Oct})_{2}$ was dried under high vacuum at $80{ }^{\circ} \mathrm{C}$ overnight before use. Toluene was distilled twice from $\mathrm{CaH}_{2}$. tBA was passed over basic alumina prior to use.

Encapsulation: Fluorescein (Aldrich), THF (inhibitor free, Aldrich), ATTO 655 (ATTO-TEC $\mathrm{GmbH}$ ), enhanced green fluorescent protein in PBS buffer (EGFP, from Biovision) were used as received.

\subsection{Polymers}

\subsubsection{Synthesis}

\subsubsection{Synthesis of difunctional photocleavable initiator $\mathrm{ONB}$}

In a round bottom flask, 5-hydroxy-2-nitrobenzyl alcohol $(0.818 \mathrm{~g}, 4.8 \mathrm{mmol})$ was dissolved in anhydrous ethyl acetate $(20 \mathrm{~mL})$. Triethylamine $(0.44 \mathrm{~mL}, 0.326 \mathrm{~g}, 4.8 \mathrm{mmol})$ was added under stirring and nitrogen flow. $\alpha$-bromoisobutyryl bromide $(0.39 \mathrm{~mL}, 0.741 \mathrm{~g}, 3.22 \mathrm{mmol})$ diluted in ethyl acetate $(5 \mathrm{~mL})$ was added dropwise via syringe pump over 90 minutes and let to react for 24 hours at room temperature. The precipitate was filtered off and washed twice with ethyl 
acetate. The combined organic phases were extracted with $0.1 \mathrm{M} \mathrm{NaHCO}_{3}(2 \mathrm{x} 50 \mathrm{~mL})$, brine $(2 \mathrm{x}$ $50 \mathrm{~mL})$ and water $(2 \mathrm{x} 50 \mathrm{~mL})$, then dried over $\mathrm{MgSO}_{4}$ and the solvent was distilled under reduced pressure. Further purification was performed using flash chromatography (TLC: $R_{f}=$ $0.45, \Delta \mathrm{R}_{\mathrm{f}}=0.17$; separation on silica gel column; 20-80\% EtOAc in hexane) to yield the desired compound as a clear orange oil; ${ }^{1} \mathrm{H}$ NMR $\left(\mathrm{CDCl}_{3}, 400 \mathrm{MHz}\right) \delta_{\mathrm{H}}(\mathrm{ppm}): 2.08\left(6 \mathrm{H}, \mathrm{s}, 2 \mathrm{xCH}_{3}\right)$, $5.06\left(2 \mathrm{H}, \mathrm{d}, \mathrm{CH}_{2}\right), 7.25$ (1H, dd, aromatic), $7.61\left(1 \mathrm{H}, \mathrm{d}\right.$, aromatic), 8.22 (1H, d, aromatic); ${ }^{13} \mathrm{C}$ NMR $\left(\mathrm{CDCl}_{3}, 400 \mathrm{MHz}\right) \delta_{\mathrm{C}}(\mathrm{ppm}): 30.8\left(2 \mathrm{xCH}_{3}\right), 55.1(\mathrm{C}-\mathrm{Br}), 62.5\left(\mathrm{CH}_{2}\right), 121.2(\mathrm{C} 3), 122.2$ (C5), 127.4 (C6), 140.4 (C2), 144.9 (C1), 155.3 (C4), $169.9(\mathrm{C}=\mathrm{O})$.

\subsubsection{Synthesis of PMCL-ONB-PAA block copolymer}

PMCL block: Ring opening polymerization was carried out under inert atmosphere and water free conditions using standard Schlenk techniques. $\mathrm{Sn}(\mathrm{Oct})_{2}(127 \mathrm{mg}, 314 \mu \mathrm{mol})$ was placed in a $25 \mathrm{~mL}$, two-neck, and round bottom flask equipped with a gas inlet and a septum. The catalyst was dried overnight under high vacuum at $80^{\circ} \mathrm{C}$. To this flask, a solution of ONB initiator (200 $\mathrm{mg}, 620 \mu \mathrm{mol})$ in $5 \mathrm{~mL}$ of dry toluene was added, followed by 3 freeze-pump-thaw cycles. Then the solution was allowed to react for $30 \mathrm{~min}$ at $90^{\circ} \mathrm{C}$. Freshly distilled $\gamma$-methyl-e-caprolactone $(8.05 \mathrm{~g}, 62 \mathrm{mmol})$ was then added to the reaction flask via syringe. The reaction mixture was then let to react for 10 hours at $90^{\circ} \mathrm{C}$. The resulting polymer solution was diluted with a small amount of THF, precipitated in $250 \mathrm{~mL}$ of cold methanol $\left(-30^{\circ} \mathrm{C}\right)$, and washed with $4 \times 50 \mathrm{~mL}$ of cold methanol. The polymer was dried overnight under high vacuum.

PtBA block: For the ATRP of tBA under oxygen free conditions, PmCL-ONB macroinitiator $\left(\mathrm{MW}=13750 \mathrm{~g} \cdot \mathrm{mol}^{-1}, \mathrm{PDI}=1.31,1.0 \mathrm{~g}, 72.7 \mu \mathrm{mol}\right)$, tert-butyl acrylate $(1.58 \mathrm{~mL}, 1.40 \mathrm{~g}, 10.9$ $\mathrm{mmol}), \mathrm{CuBr}(10.4 \mathrm{mg}, 72.7 \mu \mathrm{mol})$ and toluene $(5 \mathrm{~mL})$ were added to a three-neck, round bottom flask equipped with a refrigerant, a gas inlet, and a septum. The solution was degassed with 3 freeze-pump-thaw cycles. PMDETA $(12.6 \mathrm{mg}, 72.7 \mu \mathrm{mol})$ dissolved in $2 \mathrm{~mL}$ toluene was bubbled with $\mathrm{N}_{2}$ for 1 hour before addition via gas-purged syringe to the reaction flask. The flask was then placed in an oil bath at $75^{\circ} \mathrm{C}$. After a given reaction time the resulting polymer solution 
was exposed to air and diluted with non-degassed THF. The polymer solution was passed through a basic alumina column. After concentration, the polymer was precipitated in $100 \mathrm{~mL}$ of cold methanol/water mixture (9/1 volume) and dried under high vacuum.

Deprotection of PtBA block: In the last step, the PtBA polymer units were deprotected under mild acidolysis. In a round bottom flask, PmCL-ONB-PtBA (MW = $22000 \mathrm{~g} \cdot \mathrm{mol}^{-1}$, PDI = 1.21, $0.81 \mathrm{~g}, 1.2 \mathrm{mmol}$ of tBA units) was dissolved in dry dichloromethane $(50 \mathrm{~mL})$ and TFA (15 eq/tBA unit, $2.2 \mathrm{~g}, 19.3 \mathrm{mmol}$ ) was added. The reaction was stirred for 18 hours at room temperature, then the polymer was precipitated in $250 \mathrm{~mL}$ of cold diethyl ether. Traces of TFA were removed under high vacuum.

\subsubsection{Characterization}

\subsubsection{NMR}

Organic structures and polymers were analyzed on a bruker $400 \mathrm{MHz}$. Most of the compounds were dissolved in $\mathrm{CDCl} 3$, with TMS as an internal reference (128 scans). In the case of the amphiphilic diblock copolymers, d-THF was used.

\subsubsection{2 $\quad G P C$}

Polymer molecular weights and PDIs were estimated using a Viscotek GPCmax system equipped with a PLgel $10 \mathrm{~mm}$ column, THF as eluent (flow rate $=1 \mathrm{~mL} \cdot \mathrm{min}^{-1}$ ) at $30^{\circ} \mathrm{C}$ and using refractiveindex (RI), UV, viscosimetry, and $90^{\circ}$ angle light scattering for detection; the calibration was carried out using polystyrene standards.

\subsubsection{IR}

Fourier-transform infrared (FTIR) spectra were recorded on a Shimadzu 8400S instrument. 


\subsection{UV degradation}

Samples were exposed to UV light provided by a Hamamatsu UV spot light source LC4 (200W mercury-xenon lamp, spectral range 240 to $400 \mathrm{~nm}$ ), equipped with a fiber optic light guide. The required irradiation wavelength $(365 \mathrm{~nm})$ was isolated using a set of UV filters (UG11 and WG 360, Schott Glass, see UV CO spectra in Figure 21). Samples were placed in Quartz cuvettes, preferred to prevent any UV absorption from common glass vials. Several irradiation intensities were obtained as a function of light source to sample distance. Samples were irradiated using 20 , 200 , or $700 \mathrm{~mW} / \mathrm{cm}^{2}$, for a given amount of time.

\subsection{Self assembled structures}

\subsubsection{Preparation}

Preparation of self assembled structures for morphology studies: polymer was dissolved in a minimum amount of inhibitor free THF. We used inhibitor free THF to avoid UV light absorption by butylated hydroxytoluene (BHT). Then phosphate buffered saline (PBS) was slowly dropwise added. The $\mathrm{pH}$ of the solution was adjusted to 7.4 (physiological $\mathrm{pH}$ ) with $0.5 \mathrm{M}$ $\mathrm{NaOH}$ solution (or $\mathrm{HCl} 0.5 \mathrm{M}$ ) if needed. The solutions were allowed to equilibrate in the dark for 3 days. Samples were extruded through $1 \mu \mathrm{m}, 0.4 \mu \mathrm{m}$ and $0.2 \mu \mathrm{m}$ filters to get rid of large size aggregates. Vesicles were isolated from micellar structures via Size Exclusion Chromatography (Sepharose 2B, eluent PBS). We evaluated the final polymer concentration (i.e. after extrusion and SEC) using a fluorescence calibration curve. The fluorescence signal from the ONB linker was used to build this calibration.

\subsubsection{Characterization}

\subsubsection{DLS}

The hydrodynamic radii and size distribution of the self-assembled copolymers were measured at room temperature by dynamic light scattering (scattering angle: $90^{\circ}, 3$ measurements of $300 \mathrm{~s}$ 
each) with a commercial goniometer (ALV/CGS-8F, ALV Langen) equipped with a He:Ne linear polarized laser (JDS Uniphase, wavelength $=632.8 \mathrm{~nm}$ ). An ALV-5000/60X0 correlator was used to calculate the correlation function of the scattered light intensity, which was analyzed using the CONTIN algorithm.

\subsubsection{SLS}

For static light scattering measurements, we used solutions with different polymer concentrations (from 0.1 to $1.0 \mathrm{mg} \cdot \mathrm{mL}^{-1}$ ), at scattering angles from 40 to $150^{\circ}$ with $10^{\circ}$ angular steps. For each angle, three measurements of 100 seconds each were performed. For DLS and SLS data processing we used the ALV static \& dynamic fit and plot software (version 4.31 10/01). SLS data were processed according to the Berry-model, and cumulant analysis.

\subsubsection{TEM}

Transmission Electron Microscopy (TEM) was employed as imaging technique to visualize the self-assembled copolymer structures. 0.1 to $1.0 \mathrm{mg} \cdot \mathrm{mL}^{-1}$ solutions of polymer were placed on a copper grid (300 mesh), treated with glow discharge to make them hydrophilic. A drop of solution was placed on the grid, and the excess liquid was blotted. Then the grids were stained ( $2 \%$ uranyl acetate), followed by a rinsing step. In some cases, the rinsing step was avoided, in order to make sure that material was not lost during this operation. TEM micrographs were acquired with a Philips Morgagni 268D instrument, operated at $80 \mathrm{kV}$.

\subsection{Encapsulation and release}

\subsubsection{Preparation of loaded vesicles}

Preparation of vesicles encapsulating Fluorescein: a solution of Fluorescein in PBS buffer $(25 \mathrm{mM})$ was prepared. It was added drop by drop to a polymer solution in THF. The solution was allowed to equilibrate for 3 days in the dark. Following equilibration time, free fluorescein 
was removed by dialysis against fresh PBS buffer (for $2 \mathrm{~mL}$ of solution, 2 x800mL buffer exchange, then $800 \mathrm{~mL}$ overnight, using a $100000 \mathrm{MWCO}$ dialysis membrane). Traces of free fluorescein were removed via SEC (Sepharose 2B, PBS).

ATTO655: Similar encapsulation procedure as above, using a $25 \mu \mathrm{M}$ solution in PBS buffer. Extrusion with 1 and $0.4 \mu \mathrm{m}$ filters prior to dialysis, but without performing SEC.

$\underline{e G F P}$ : Similar encapsulation procedure, with a $3 \mu \mathrm{M}$ eGFP solution in PBS buffer. Extrusion with 1 and $0.4 \mu \mathrm{m}$ filters prior to dialysis (with $300000 \mathrm{MWCO}$ membrane), but without performing SEC.

\subsubsection{Fluorimetry}

Release of encapsulated compound: The amount of released dye was followed at room temperature using fluorescence spectroscopy (on a Perkin Elmer, LS55). Irradiated samples were placed in Quartz cuvettes and directly analyzed. The excitation wavelength was set at 494nm and the emission spectra were recorded from 450 to $650 \mathrm{~nm}$. The instrument was used in scan mode, with excitation slit set to $10 \mathrm{~nm}$ and emission slit set to $3 \mathrm{~nm}$.

\subsubsection{FCS}

Fluorescence correlation spectroscopy (FCS) was performed with an inverted confocal fluorescence laser scanning microscope LSM 510 META/Confocor2 (Zeiss, Germany) equipped with an argon laser (for 405, 458, 477, 488 and $514 \mathrm{~nm}$ ), and two helium-neon lasers (543 and $633 \mathrm{~nm}$ ) as excitation sources. For FCS, $15 \mu \mathrm{L}$ solution were applied to the glass surface of a cover glass (Huber \& Co. AG, Switzerland) positioned on the xy-stage of the microscope. The excitation laser beam and the fluorescence emission passed through the same objective. The fluorescence signal was detected by highly sensitive avalanche photo diodes. Fluorescence intensity fluctuations were processed in terms of an autocorrelation function. 


\section{References}

1. Förster, S. and T. Plantenberg, From Self-Organizing Polymers to Nanohybrid and Biomaterials. Angewandte Chemie International Edition, 2002. 41(5): p. 688-714.

2. Tanford, C., The hydrophobic effect and the organization of living matter. Science, 1978. 200(4345): p. 1012-1018.

3. Hayward, R.C. and D.J. Pochan, Tailored Assemblies of Block Copolymers in Solution: It Is All about the Process. Macromolecules, 2010. 43(8): p. 3577-3584.

4. Choucair, A. and A. Eisenberg, Control of amphiphilic block copolymer morphologies using solution conditions. The European Physical Journal E: Soft Matter and Biological Physics, 2003. 10(1): p. 37-44.

5. Holder, S.J. and N.A.J.M. Sommerdijk, New micellar morphologies from amphiphilic block copolymers: disks, toroids and bicontinuous micelles. Polymer Chemistry, 2011.

6. Antonietti, M. and S. Förster, Vesicles and Liposomes: A Self-Assembly Principle Beyond Lipids. Advanced Materials, 2003. 15(16): p. 1323-1333.

7. Guida, V., Thermodynamics and kinetics of vesicles formation processes. Advances in Colloid and Interface Science, 2010. 161(1-2): p. 77-88.

8. Discher, D.E. and F. Ahmed, POLYMERSOMES. Annual Review of Biomedical Engineering, 2006. 8(1): p. 323-341.

9. Luo, L. and A. Eisenberg, Langmuir, 2001. 17: p. 6804.

10. Terreau, O., L. Luo, and A. Eisenberg, Effect of Poly(acrylic acid) Block Length Distribution on Polystyrene-b-Poly(acrylic acid) Aggregates in Solution. 1. Vesicles. Langmuir, 2003. 19(14): p. 5601-5607.

11. Kita-Tokarczyk, K. and W. Meier, Biomimetic Block Copolymer Membranes. CHIMIA International Journal for Chemistry, 2008. 62: p. 820-825.

12. Uneyama, T., Density functional simulation of spontaneous formation of vesicle in block copolymer solutions. The Journal of Chemical Physics, 2007. 126(11): p. 114902-17.

13. Du, J. and Y. Chen, Preparation of Organic/Inorganic Hybrid Hollow Particles Based on Gelation of Polymer Vesicles. Macromolecules, 2004. 37(15): p. 5710-5716.

14. Adams, D.J., et al., Impact of mechanism of formation on encapsulation in block copolymer vesicles. Journal of Controlled Release, 2008. 128(2): p. 165-170.

15. Discher, D.E. and A. Eisenberg, Polymer Vesicles. Science, 2002. 297(5583): p. 967-973.

16. Meng, F., Z. Zhong, and J. Feijen, Stimuli-Responsive Polymersomes for Programmed Drug Delivery. Biomacromolecules, 2009. 10(2): p. 197-209.

17. Napoli, A., et al., Oxidation-responsive polymeric vesicles. Nat Mater, 2004. 3(3): p. 183189. 
18. Onaca, O., et al., Stimuli-Responsive Polymersomes as Nanocarriers for Drug and Gene Delivery. Macromolecular Bioscience, 2009. 9(2): p. 129-139.

19. Bermudez, H., et al., Molecular Weight Dependence of Polymersome Membrane Structure, Elasticity, and Stability. Macromolecules, 2002. 35(21): p. 8203-8208.

20. Battaglia, G. and A.J. Ryan, Bilayers and Interdigitation in Block Copolymer Vesicles. Journal of the American Chemical Society, 2005. 127(24): p. 8757-8764.

21. Discher, B.M., et al., Polymersomes: Tough Vesicles Made from Diblock Copolymers. Science, 1999. 284(5417): p. 1143-1146.

22. LoPresti, C., et al., Polymersomes: nature inspired nanometer sized compartments. Journal of Materials Chemistry, 2009. 19(22): p. 3576-3590.

23. Kukula, H., et al., The Formation of Polymer Vesicles or "Peptosomes" by Polybutadiene-block-poly(l-glutamate)s in Dilute Aqueous Solution. Journal of the American Chemical Society, 2002. 124(8): p. 1658-1663.

24. Zhang, L. and A. Eisenberg, Multiple Morphologies of "Crew-Cut" Aggregates of Polystyrene-b-poly(acrylic acid) Block Copolymers. Science, 1995. 268(5218): p. 17281731.

25. Nardin, C., et al., Polymerized ABA Triblock Copolymer Vesicles. Langmuir, 2000. 16(3): p. 1035-1041.

26. Wittemann, A., T. Azzam, and A. Eisenberg, Biocompatible Polymer Vesicles from Biamphiphilic Triblock Copolymers and Their Interaction with Bovine Serum Albumin. Langmuir, 2007. 23(4): p. 2224-2230.

27. Zhao, W., et al., ABC Triblock Copolymer Vesicles with Mesh-Like Morphology. ACS Nano, 2011. 5(1): p. 486-492.

28. del Barrio, J., et al., Self-Assembly of Linear-Dendritic Diblock Copolymers: From Nanofibers to Polymersomes. Journal of the American Chemical Society, 2010. 132(11): p. 3762-3769.

29. Shi, Z., Y. Zhou, and D. Yan, Facile Fabrication of pH-Responsive and SizeControllable Polymer Vesicles From a Commercially Available Hyperbranched Polyester. Macromolecular Rapid Communications, 2008. 29(5): p. 412-418.

30. van Hest, J.C.M., et al., Polystyrene-Dendrimer Amphiphilic Block Copolymers with a Generation-Dependent Aggregation. Science, 1995. 268(5217): p. 1592-1595.

31. Lee, H.J., et al., Biodegradable Polymersomes from Poly(2-hydroxyethyl aspartamide) Grafted with Lactic Acid Oligomers in Aqueous Solution. Macromolecules, 2006. 39(15): p. 4938-4940.

32. Rakhmatullina, E., Methacrylate-based amphiphilic block copolymers in solution and at surfaces: synthesis, characterization and self-assembly, in Department of Chemistry. 2008, University of Basel: Basel. p. 148. 
33. Yu, Y., L. Zhang, and A. Eisenberg, Multiple Morphologies of Crew-Cut Aggregates of Polybutadiene-b-poly(acrylic acid) Diblocks with Low Tg Cores. Langmuir, 1997. 13(9): p. 2578-2581.

34. Jacquin, M., et al., Chemical analysis and aqueous solution properties of charged amphiphilic block copolymers PBA-b-PAA synthesized by MADIX®. Journal of Colloid and Interface Science, 2007. 316(2): p. 897-911.

35. Colombani, O., et al., Structure of Micelles of Poly(n-butyl acrylate)-block-poly(acrylic acid) Diblock Copolymers in Aqueous Solution. Macromolecules, 2007. 40(12): p. 43514362.

36. Zhang, Q., E.E. Remsen, and K.L. Wooley, Shell Cross-Linked Nanoparticles Containing Hydrolytically Degradable, Crystalline Core Domains. Journal of the American Chemical Society, 2000. 122(15): p. 3642-3651.

37. Henselwood, F. and G. Liu, Water-Soluble Nanospheres of Poly(2-cinnamoylethyl methacrylate)-block-poly(acrylic acid). Macromolecules, 1997. 30(3): p. 488-493.

38. Huang, H., et al., Nanocages Derived from Shell Cross-Linked Micelle Templates. Journal of the American Chemical Society, 1999. 121(15): p. 3805-3806.

39. Qi, K., et al., Determination of the Bioavailability of Biotin Conjugated onto Shell CrossLinked (SCK) Nanoparticles. Journal of the American Chemical Society, 2004. 126(21): p. 6599-6607.

40. Braun, J., Phase Diagrams and Applications of Amphiphilic Block Copolymers in Aqueous Solutions, in Department of Chemistry. 2011, University of Basel: Basel. p. 99.

41. Zupancich, J.A., F.S. Bates, and M.A. Hillmyer, Aqueous Dispersions of Poly(ethylene

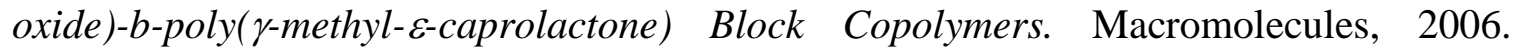
39(13): p. 4286-4288.

42. Le Meins, J.F., O. Sandre, and S. Lecommandoux, Recent trends in the tuning of polymersomes' membrane properties. Eur. Phys. J. E, 2011. 34(2): p. 14.

43. Schmaljohann, D., Thermo- and pH-responsive polymers in drug delivery. Advanced Drug Delivery Reviews, 2006. 58(15): p. 1655-1670.

44. Gil, E.S. and S.M. Hudson, Stimuli-reponsive polymers and their bioconjugates. Progress in Polymer Science, 2004. 29(12): p. 1173-1222.

45. Delcea, M., H. Möhwald, and A.G. Skirtach, Stimuli-responsive LbL capsules and nanoshells for drug delivery. Advanced Drug Delivery Reviews, 2011. In Press, Corrected Proof.

46. Liechty, W.B., et al., Polymers for Drug Delivery Systems. Annual Review of Chemical and Biomolecular Engineering, 2010. 1(1): p. 149-173.

47. Liu, F. and A. Eisenberg, Preparation and $\mathrm{pH}$ Triggered Inversion of Vesicles from Poly(acrylic Acid)-block-Polystyrene-block-Poly(4-vinyl Pyridine). Journal of the American Chemical Society, 2003. 125(49): p. 15059-15064. 
48. Du, J., et al., pH-Sensitive Vesicles Based on a Biocompatible Zwitterionic Diblock Copolymer. Journal of the American Chemical Society, 2005. 127(51): p. 17982-17983.

49. Rodríguez-Hernández, J. and S. Lecommandoux, Reversible Inside-Out Micellization of pH-responsive and Water-Soluble Vesicles Based on Polypeptide Diblock Copolymers. Journal of the American Chemical Society, 2005. 127(7): p. 2026-2027.

50. Lin, S., et al., An Acid-Labile Block Copolymer of PDMAEMA and PEG as Potential Carrier for Intelligent Gene Delivery Systems. Biomacromolecules, 2007. 9(1): p. 109115.

51. Li, Y., B.S. Lokitz, and C.L. McCormick, Thermally Responsive Vesicles and Their Structural "Locking" through Polyelectrolyte Complex Formation. Angewandte Chemie International Edition, 2006. 45(35): p. 5792-5795.

52. Qin, S., et al., Temperature-Controlled Assembly and Release from Polymer Vesicles of Poly(ethylene oxide)-block- poly(N-isopropylacrylamide). Advanced Materials, 2006. 18(21): p. 2905-2909.

53. Napoli, A., et al., Glucose-oxidase Based Self-Destructing Polymeric Vesicles. Langmuir, 2004. 20(9): p. 3487-3491.

54. Cerritelli, S., D. Velluto, and J.A. Hubbell, PEG-SS-PPS: Reduction-Sensitive Disulfide Block Copolymer Vesicles for Intracellular Drug Delivery. Biomacromolecules, 2007. 8(6): p. 1966-1972.

55. Alvarez-Lorenzo, C., L. Bromberg, and A. Concheiro, Light-sensitive Intelligent Drug Delivery Systems $†$. Photochemistry and Photobiology, 2009. 85(4): p. 848-860.

56. Du, J. and R.K. O'Reilly, Advances and challenges in smart and functional polymer vesicles. Soft Matter, 2009. 5(19): p. 3544-3561.

57. Li, M.-H. and P. Keller, Stimuli-responsive polymer vesicles. Soft Matter, 2009. 5(5): p. 927-937.

58. Stuart, M.A.C., et al., Emerging applications of stimuli-responsive polymer materials. Nat Mater, 2010. 9(2): p. 101-113.

59. Chandra, B., et al., Formulation of photocleavable liposomes and the mechanism of their content release. Organic \& Biomolecular Chemistry, 2006. 4(9): p. 1730-1740.

60. Bisby, R.H., C. Mead, and C.G. Morgan, Wavelength-Programmed Solute Release from Photosensitive Liposomes. Biochemical and Biophysical Research Communications, 2000. 276(1): p. 169-173.

61. Yagai, S., T. Karatsu, and A. Kitamura, Photocontrollable Self-Assembly. Chemistry - A European Journal, 2005. 11(14): p. 4054-4063.

62. Troutman, T.S., S.J. Leung, and M. Romanowski, Light-Induced Content Release from Plasmon-Resonant Liposomes. Advanced Materials, 2009. 21(22): p. 2334-2338. 
63. Bisby, R.H., C. Mead, and C.G. Morgan, Active Uptake of Drugs into Photosensitive Liposomes and Rapid Release on UV Photolysis. Photochemistry and Photobiology, 2000. 72(1): p. 57-61.

64. Jiang, J., et al., Toward Photocontrolled Release Using Light-Dissociable Block Copolymer Micelles. Macromolecules, 2006. 39(13): p. 4633-4640.

65. Zhao, Y., Photocontrollable block copolymer micelles: what can we control? Journal of Materials Chemistry, 2009. 19(28): p. 4887-4895.

66. Lee, H.-i., et al., Light-Induced Reversible Formation of Polymeric Micelles. Angewandte Chemie International Edition, 2007. 46(14): p. 2453-2457.

67. Liu, X. and M. Jiang, Optical Switching of Self-Assembly: Micellization and MicelleHollow-Sphere Transition of Hydrogen-Bonded Polymers. Angewandte Chemie International Edition, 2006. 45(23): p. 3846-3850.

68. Jiang, Y., et al., Reversible Self-Organization of a UV-Responsive PEG-Terminated Malachite Green Derivative: Vesicle Formation and Photoinduced Disassembly. Langmuir, 2007. 23(7): p. 4029-4034.

69. Robbins, G.P., et al., Photoinitiated Destruction of Composite Porphyrinâ^’Protein Polymersomes. Journal of the American Chemical Society, 2009. 131(11): p. 3872-3874.

70. Yuan, X., K. Fischer, and W. Schartl, Photocleavable Microcapsules Built from Photoreactive Nanospheres. Langmuir, 2005. 21(20): p. 9374-9380.

71. Han, K., et al., Reversible Photocontrolled Swelling-Shrinking Behavior of Micron Vesicles Self-Assembled from Azopyridine-Containing Diblock Copolymer. Macromolecular Rapid Communications, 2008. 29(23): p. 1866-1870.

72. Lin, L., et al., UV-Responsive Behavior of Azopyridine-Containing Diblock Copolymeric Vesicles: Photoinduced Fusion, Disintegration and Rearrangement. Macromolecular Rapid Communications, 2009. 30(13): p. 1089-1093.

73. Mabrouk, E., et al., Bursting of sensitive polymersomes induced by curling. Proceedings of the National Academy of Sciences, 2009. 106(18): p. 7294-7298.

74. Ercole, F., T.P. Davis, and R.A. Evans, Photo-responsive systems and biomaterials: photochromic polymers, light-triggered self-assembly, surface modification, fluorescence modulation and beyond. Polymer Chemistry, 2010. 1(1): p. 37-54.

75. Koňák, Č., et al., Photoregulated Association of Water-Soluble Copolymers with Spirobenzopyran-Containing Side Chains. Macromolecules, 1997. 30(18): p. 5553-5556.

76. Babin, J., et al., A New Two-Photon-Sensitive Block Copolymer Nanocarrier. Angewandte Chemie International Edition, 2009. 48(18): p. 3329-3332.

77. Jiang, X., et al., Multiple Micellization and Dissociation Transitions of Thermo- and Light-Sensitive Poly(ethylene oxide)-b-poly(ethoxytri(ethylene glycol) acrylate-co-onitrobenzyl acrylate) in Water. Macromolecules, 2008. 41(7): p. 2632-2643. 
78. Tong, X., et al., How Can Azobenzene Block Copolymer Vesicles Be Dissociated and Reformed by Light? The Journal of Physical Chemistry B, 2005. 109(43): p. 2028120287.

79. Katz, J.S., et al., Modular Synthesis of Biodegradable Diblock Copolymers for Designing Functional Polymersomes. Journal of the American Chemical Society, 2010. 132(11): p. 3654-3655.

80. Thompson, S., et al., Photocleavable Nitrobenzyl-Protein Conjugates. Biochemical and Biophysical Research Communications, 1994. 201(3): p. 1213-1219.

81. Adams, S.R. and R.Y. Tsien, Controlling Cell Chemistry with Caged Compounds. Annual Review of Physiology, 1993. 55(1): p. 755-784.

82. Kang, M. and B. Moon, Synthesis of Photocleavable Poly(styrene-block-ethylene oxide) and Its Self-Assembly into Nanoporous Thin Films. Macromolecules, 2009. 42(1): p. 455458.

83. Schumers, J.-M., J.-F. Gohy, and C.-A. Fustin, A versatile strategy for the synthesis of block copolymers bearing a photocleavable junction. Polymer Chemistry, 2010: p. --

84. Schumers, J.-M., J.-F. Gohy, and C.-A. Fustin, A versatile strategy for the synthesis of block copolymers bearing a photocleavable junction. Polymer Chemistry, 2010. 1(2): p. 161-163.

85. Cao, M., et al., Cleavage of polystyrene-b-poly(ethylene oxide) block copolymers with a trithiocarbonate linkage in solutions. Journal of Polymer Science Part A: Polymer Chemistry, 2010. 48(17): p. 3834-3840.

86. Ryu, J.-H., et al., Highly Ordered Gold Nanotubes Using Thiols at a Cleavable Block Copolymer Interface. Journal of the American Chemical Society, 2009. 131(29): p. 98709871.

87. Hartmann, L., et al., Sequence Positioning of Disulfide Linkages to Program the Degradation of Monodisperse Poly(amidoamines). Macromolecules, 2007. 40(22): p. 7771-7776.

88. Klaikherd, A., S. Ghosh, and S. Thayumanavan, A Facile Method for the Synthesis of Cleavable Block Copolymers from ATRP-Based Homopolymers. Macromolecules, 2007. 40(24): p. 8518-8520.

89. Yurt, S., et al., Scission of Diblock Copolymers into Their Constituent Blocks. Macromolecules, 2006. 39(5): p. 1670-1672.

90. Sun, H., et al., Biodegradable micelles with sheddable poly(ethylene glycol) shells for triggered intracellular release of doxorubicin. Biomaterials, 2009. 30(31): p. 6358-6366.

91. Goldbach, J.T., T.P. Russell, and J. Penelle, Synthesis and Thin Film Characterization of Poly(styrene-block-methyl methacrylate) Containing an Anthracene Dimer Photocleavable Junction Point. Macromolecules, 2002. 35(11): p. 4271-4276.

92. Sivanandan, K., et al., High Surface Area Poly(3-hexylthiophenes) Thin Films from Cleavable Graft Copolymers. Macromolecules, 2009. 43(1): p. 233-241. 
93. Bernaerts, K.V. and F.E. Du Prez, Dual/heterofunctional initiators for the combination of mechanistically distinct polymerization techniques. Progress in Polymer Science, 2006. 31(8): p. 671-722.

94. Wenxin, W., et al., The Use of E-Caprolactone as a Polymerizable Solvent for the Atom Transfer Radical Polymerization of MMA at Low Temperature. Macromolecular Chemistry and Physics, 2002. 203(7): p. 968-974.

95. Nasser-Eddine, M., et al., Controlled one-step synthesis of a diblock copolymer. European Polymer Journal, 2005. 41(2): p. 313-318.

96. Jakubowski, W. and K. Matyjaszewski, New Segmented Copolymers by Combination of Atom Transfer Radical Polymerization and Ring Opening Polymerization. Macromolecular Symposia, 2006. 240(1): p. 213-223.

97. Tao, L., B. Luan, and C.-y. Pan, Block and star block copolymers by mechanism transformation. VIII Synthesis and characterization of triblock poly(LLA-b-St-b-MMA) by combination of ATRP and ROP. Polymer, 2003. 44(4): p. 1013-1020.

98. Likhitsup, A., A. Parthiban, and C.L.L. Chai, Combining atom-transfer radical polymerization and ring-opening polymerization through bifunctional initiators derived from hydroxy benzyl alcohol-preparation and characterization of initiators, macroinitiators, and block copolymers. Journal of Polymer Science Part A: Polymer Chemistry, 2008. 46(1): p. 102-116.

99. Ydens, I., et al., Combining ATRP of Methacrylates and ROP of L,L-Dilactide and $\varepsilon$ Caprolactone. Macromolecular Chemistry and Physics, 2003. 204(1): p. 171-179.

100. Goodwin, A.P., et al., Synthetic Micelle Sensitive to IR Light via a Two-Photon Process. Journal of the American Chemical Society, 2005. 127(28): p. 9952-9953.

101. Aujard, I., et al., o-Nitrobenzyl Photolabile Protecting Groups with Red-Shifted Absorption: Syntheses and Uncaging Cross-Sections for One- and Two-Photon Excitation. Chemistry - A European Journal, 2006. 12(26): p. 6865-6879.

102. Marinzi, C., et al., An o-nitrobenzyl scaffold for peptide ligation: synthesis and applications. Bioorganic \& Medicinal Chemistry, 2004. 12(10): p. 2749-2757.

103. Piggott, A.M. and P. Karuso, Synthesis of a new hydrophilic o-nitrobenzyl photocleavable linker suitable for use in chemical proteomics. Tetrahedron Letters, 2005. 46(47): p. 8241-8244.

104. Whitehouse, D.L., S.N. Savinov, and D.J. Austin, An improved synthesis and selective coupling of a hydroxy based photolabile linker for solid phase organic synthesis. Tetrahedron Letters, 1997. 38(45): p. 7851-7852.

105. Wang, W., et al., The Use of E-Caprolactone as a Polymerizable Solvent for the Atom Transfer Radical Polymerization of MMA at Low Temperature. Macromolecular Chemistry and Physics, 2002. 203(7): p. 968-974.

106. Odian, G., Ring-Opening Polymerization, in Principles of Polymerization, WileyInterscience, Editor. 2004. 
107. Sadykov, R., Chemically induced dynamic nuclei polarization and free radicals in the reaction of triethylaluminum with nitrobenzene. Russian Chemical Bulletin, 1998. 47(10): p. 1924-1927.

108. Storey, R.F. and J.W. Sherman, Kinetics and Mechanism of the Stannous OctoateCatalyzed Bulk Polymerization of Î -Caprolactone. Macromolecules, 2002. 35(5): p. 1504-1512.

109. Kricheldorf, H.R., A. Stricker, and D. Langanke, Polylactones, 52. Tin Carboxylates as Initiators of $\varepsilon$-Caprolactone. Macromolecular Chemistry and Physics, 2001. 202(15): p. 2963-2970.

110. Kowalski, A., A. Duda, and S. Penczek, Kinetics and mechanism of cyclic esters polymerization initiated with tin(II) octoate, 1. Polymerization of $\varepsilon$-caprolactone. Macromolecular Rapid Communications, 1998. 19(11): p. 567-572.

111. Trollsas, M., et al., Highly Branched Block Copolymers: Design, Synthesis, and Morphology. Macromolecules, 1999. 32(15): p. 4917-4924.

112. Adam, K., D. Andrzej, and P. Stanislaw, Kinetics and mechanism of cyclic esters polymerization initiated with tin(II) octoate, 1. Polymerization of $\langle I\rangle$ eta $\langle/ I\rangle$ caprolactone. Macromolecular Rapid Communications, 1998. 19(11): p. 567-572.

113. Tsarevsky, N.V. and K. Matyjaszewski, Controlled Synthesis of Polymers with Ionic or Ionizable Groups Using Atom Transfer Radical Polymerization. ACS Symposium Series, 2006. 937: p. 79-94.

114. Tsarevsky, N.V. and K. Matyjaszewski, â€œGreenâ€• Atom Transfer Radical Polymerization:â€\%o From Process Design to Preparation of Well-Defined Environmentally Friendly Polymeric Materials. Chemical Reviews, 2007. 107(6): p. 2270-2299.

115. Qinggao, M. and L.W. Karen, The preparation of t-butyl acrylate, methyl acrylate, and styrene block copolymers by atom transfer radical polymerization: Precursors to amphiphilic and hydrophilic block copolymers and conversion to complex nanostructured materials. Journal of Polymer Science Part A: Polymer Chemistry, 2000. 38(S1): p. 4805-4820.

116. Östmark, E., D. Nyström, and E. Malmström, Unimolecular Nanocontainers Prepared by ROP and Subsequent ATRP from Hydroxypropylcellulose. Macromolecules, 2008. 41(12): p. 4405-4415.

117. Céline, T., et al., Amphiphilic Multi-Arm Star-Block Copolymers for Encapsulation of Fragrance Molecules. Macromolecular Chemistry and Physics, 2007. 208(2): p. 131-145.

118. Frederick, J.E., H.E. Snell, and E.K. Haywood, SOLAR ULTRAVIOLET RADIATION AT THE EARTH'S SURFACE. Photochemistry and Photobiology, 1989. 50(4): p. 443-450.

119. Blanc, A. and C.G. Bochet, Isotope Effects in Photochemistry. 1. o-Nitrobenzyl Alcohol Derivatives. Journal of the American Chemical Society, 2004. 126(23): p. 7174-7175. 
120. Wieboldt, R., et al., Synthesis and Characterization of Photolabile o-Nitrobenzyl Derivatives of Urea. The Journal of Organic Chemistry, 2002. 67(25): p. 8827-8831.

121. King, I.R. and G.W. Kirby, Nuclear magnetic resonance and mass spectra of nitrosobenzene derivatives. Journal of the Chemical Society C: Organic, 1966: p. 13341336.

122. Barltrop, J.A. and N.J. Bunce, Organic photochemistry. Part VIII. The photochemical reduction of nitro-compounds. Journal of the Chemical Society C: Organic, 1968: p. 1467-1474.

123. Adams, D.J., et al., On the mechanism of formation of vesicles from poly(ethylene oxide)block-poly(caprolactone) copolymers. Soft Matter, 2009. 5(16): p. 3086-3096.

124. Ghoroghchian, P.P., et al., Bioresorbable Vesicles Formed through Spontaneous SelfAssembly of Amphiphilic Poly(ethylene oxide)-block-polycaprolactone. Macromolecules, 2006. 39(5): p. 1673-1675.

125. Lifeng, Z., R.J. Barlow, and A. Eisenberg, Scaling relations and coronal dimensions in aqueous block polyelectrolyte micelles. Macromolecules, 1995. 28(18): p. 6055-6066.

126. Opsteen, J.A., J.J.L.M. Cornelissen, and J.C.M.v. Hest, Block copolymer vesicles. Pure and Applied Chemistry, 2004. 76(7-8): p. 1309-1319.

127. Shen, H. and A. Eisenberg, Block Length Dependence of Morphological Phase Diagrams of the Ternary System of PS-b-PAA/Dioxane/H2O. Macromolecules, 2000. 33(7): p. 2561-2572.

128. Safran, S.A., P. Pincus, and D. Andelman, Theory of Spontaneous Vesicle Formation in Surfactant Mixtures. Science, 1990. 248(4953): p. 354-356.

129. Hotz, J. and W. Meier, Vesicle-Templated Polymer Hollow Spheres. Langmuir, 1998. 14(5): p. 1031-1036.

130. Kakizawa, Y., A. Harada, and K. Kataoka, Environment-Sensitive Stabilization of Coreâ' Shell Structured Polyion Complex Micelle by Reversible Cross-Linking of the Core through Disulfide Bond. Journal of the American Chemical Society, 1999. 121(48): p. 11247-11248.

131. Griffin, D.R., J.T. Patterson, and A.M. Kasko, Photodegradation as a mechanism for controlled drug delivery. Biotechnology and Bioengineering, 2010. 107(6): p. 1012-1019.

132. Christian, D.A., et al., Polymersome carriers: From self-assembly to siRNA and protein therapeutics. European Journal of Pharmaceutics and Biopharmaceutics, 2009. 71(3): p. 463-474.

133. Lomas, H., et al., Biomimetic $p H$ Sensitive Polymersomes for Efficient DNA Encapsulation and Delivery. Advanced Materials, 2007. 19(23): p. 4238-4243.

134. Stoenescu, R., A. Graff, and W. Meier, Asymmetric ABC-Triblock Copolymer Membranes Induce a Directed Insertion of Membrane Proteins. Macromolecular Bioscience, 2004. 4(10): p. 930-935. 
135. Kumar, M., et al., Highly permeable polymeric membranes based on the incorporation of the functional water channel protein Aquaporin Z. Proceedings of the National Academy of Sciences, 2007. 104(52): p. 20719-20724.

136. Kim, K.T., et al., A Polymersome Nanoreactor with Controllable Permeability Induced by Stimuli-Responsive Block Copolymers. Advanced Materials, 2009. 21(27): p. 27872791.

137. Cheng, Z., D.L.J. Thorek, and A. Tsourkas, Porous Polymersomes with Encapsulated Gd-Labeled Dendrimers as Highly Efficient MRI Contrast Agents. Advanced Functional Materials, 2009. 19(23): p. 3753-3759.

138. Dong, W., et al., pH-responsive self-assembly of carboxyl-terminated hyperbranched polymers. Physical Chemistry Chemical Physics, 2007. 9(10): p. 1255-1262.

139. Hawker, C.J., K.L. Wooley, and J.M.J. Frechet, Unimolecular micelles and globular amphiphiles: dendritic macromolecules as novel recyclable solubilization agents. Journal of the Chemical Society, Perkin Transactions 1, 1993(12): p. 1287-1297.

140. Benaglia, M., et al., Polymeric micelles using pseudo-amphiphilic block copolymers and their cellular uptake. Journal of Materials Chemistry, 2011. 21(8): p. 2555-2562.

141. Liu, S., et al., Self-Assembly of Narrowly Distributed Carboxy-Terminated Linear Polystyrene Chains in Water via Microphase Inversion. Macromolecules, 2000. 33(23): p. 8640-8643.

142. Celie, P.H.N., et al., UV-Induced Ligand Exchange in MHC Class I Protein Crystals. Journal of the American Chemical Society, 2009. 131(34): p. 12298-12304.

143. Rigler, P. and W. Meier, Encapsulation of Fluorescent Molecules by Functionalized Polymeric Nanocontainers:â€\%o Investigation by Confocal Fluorescence Imaging and Fluorescence Correlation Spectroscopy. Journal of the American Chemical Society, 2005. 128(1): p. 367-373.

144. Petráaek, Z.k. and P. Schwille, Precise Measurement of Diffusion Coefficients using Scanning Fluorescence Correlation Spectroscopy. Biophysical journal, 2008. 94(4): p. 1437-1448.

145. Ahmed, F. and D.E. Discher, Self-porating polymersomes of PEG-PLA and PEG-PCL: hydrolysis-triggered controlled release vesicles. Journal of Controlled Release, 2004. 96(1): p. 37-53.

146. Du, J. and S.P. Armes, pH-Responsive Vesicles Based on a Hydrolytically Self-CrossLinkable Copolymer. Journal of the American Chemical Society, 2005. 127(37): p. 12800-12801.

147. Kim, B.-S., S.W. Park, and P.T. Hammond, Hydrogen-Bonding Layer-by-LayerAssembled Biodegradable Polymeric Micelles as Drug Delivery Vehicles from Surfaces. ACS Nano, 2008. 2(2): p. 386-392. 


\section{Acknowledgments}

I was told before I joined this group, that if I enjoyed working autonomously, I would have the time of my life. Well, indeed, I had a great time. And certainly, Prof. Dr. Wolfgang Meier, who supervised me along these years, is no stranger to that. I especially appreciated his soft guidance and enthusiasm toward new ideas.

I thank Dr. Violeta Malinova, for she, along with Prof. Dr. Wolfgang Meier, gave me the chance to work on a very challenging and exciting project. She helped me all along, whether with chemistry or with writing, and I learned a great deal working with her.

I thank PD Dr. Cornelia Palivan, who helped me a lot with writing, and to be my coreferee, meaning to spend a lot of her time reviewing my thesis. This thank also goes, and for the same reasons, to Marc and Mihaela.

I want to thank all the present and former members of the group. Like I said, it was very enjoyable to work at Klingelberstrasse 80 for all those years, both on personal and professional levels. Special thanks go to Stefan, for his friendship from the first day, and for supporting me in the quest of our first 4000er in the Alps, to Jörg for all the nice talks and good laughs, to Sindhu for scientific discussions, but mostly for discussions way beyond science, to Vimal and Thomas who welcomed me several times after wild parties in Basel, to Ozana for being who she is and for invaluable discussions, and to Sven for he is sort of a pillar in this group.

I also thank Gaby, Vesna, Pavel, Emma, Kate, and Dirk for their great help regarding technical matters, measurements, and scientific discussions.

I want to thank my family, especially my parents who, since many years, gave me invaluable advice, which always guided me toward the hard path. There is nothing more rewarding than to finally reach a summit...

Last but not least, I owe a very special thank to my wife, Marine: thanks for giving me confidence and for the countless encouragements in the hard times, thanks for your patience, and thanks for believing in me! 


\section{Curriculum Vitae}

\section{Etienne CABANE}

Badenerstrasse 736

CH-8048 Zurich

푱 $+41(0) 774480082$

etienne.cabane@unibas.ch

etienne.cabane@gmail.com

Date of birth: 26/02/82

French (B permit)

\section{EDUCATION}

2007 - current, PhD in physical chemistry

UNIVERSITY OF BASEL, Switzerland

Photoresponsive supramolecular self assemblies and their use as triggerable nanocarriers (funded by the Swiss National Science Foundation).

2005 - 2007, MSc in materials science

UNIVERSITY OF NEW HAMPSHIRE, USA

Nanoparticle oral mediated delivery of Insulin (funded by Bentley Pharmaceuticals Inc.).

2002 - 2006, Chemistry and chemical engineering diploma-equivalent to a MSc

CPE LyON, France 


\section{RESEARCH EXPERIENCE}

Nov 07 - current, Research Assistant

Department of Chemistry, UNIVERSITY OF BASEL, Switzerland.

- Synthesized new photosensitive molecules and new photocleavable block copolymers. Studied self-assembly of synthesized polymers and their potential as light sensitive drug nanocarriers.

- Work published in peer reviewed journals and presented at the Europolymer Conference 2010.

- Delivered analytical chemistry hands-on classes to chemistry and pharmacy students (bachelor level) during 6 semesters.

- Designed and delivered a new NMR practical class for chemistry students at bachelor level.

Sep 05 - Aug 07, Research Assistant

Nanostructured Polymers Research Center, UNIVERSITY OF NEW HAMPSHIRE, USA.

- Synthesized biocompatible and biodegradable triblock copolymers. Studied self-assembly, encapsulation, and release of insulin using those polymers.

- Work presented in front of Bentley Pharmaceuticals decision board.

- Designed a series of didactic experiments for the delivery of a new class of science for nonscientific bachelor students (Science of stuff).

Jan 05 - Sep 05, Research Scholar

Nanostructured Polymers Research Center, UNIVERSITY OF NEW HAMPSHIRE, USA.

- Researched on the development of a new transparent antifouling coating. Synthesized polymeric core-shell particles encapsulating a biocide. Studied film formation and biocidal properties of the obtained coatings (funded by NOAA and Rohm and Haas).

- Results published in peer reviewed journal and presented at the $230^{\text {th }}$ National ACS Meeting.

Jul 04 - Dec 04, Visiting Scientist

LRMH (research institute on cultural heritage) / MPL, ÉCOLE CENTRALE PARIS, France.

- Researched on ancient gilded brasses. Studied mercury gilding technique and investigated corrosion related degradations of ancient gilded bronzes.

- Proposed a preservation technique.

Mar 04 - Jun 04, Visiting Scientist

LMOPS (Laboratory for Organic Materials with Specific Properties), CNRS, France

- Synthesized graft block copolymers for microelectronic applications. 


\section{RELEVANT SKILLS}

\section{Research:}

- experience in designing, planning, and performing experiments

- interpreting and reporting results

- writing reports and publications

- responsible for equipments and coordinating lab maintenance

\section{Experimental skills:}

- polymer chemistry: experience in several polymerization techniques (FRP, ATRP, ROP), optimization of synthesis routes, synthesis of block copolymers, stimuliresponsive systems ( $\mathrm{pH}$, Light, enzymes), biodegradable and biocompatible polymers

- polymer characterization: separation techniques (GPC, SEC, HPLC, GC-MS), spectroscopy (NMR, FT-IR, UV-Vis, Fluorescence, FCS), calorimetry (DSC, TGA)

- Supramolecular chemistry: self-assembly methods for amphiphilic copolymers, encapsulation techniques, particle size and shape analysis (light scattering), morphology analysis (microscopy techniques: TEM, Cryo-TEM, and SEM)

\section{Training and teaching:}

- supervising student projects

- collaborating with professors for teaching projects

- training collaborators on instruments and software

\section{Specialized coursework:}

- polymer synthesis, characterization, and processing

- organic and inorganic chemistry

- thermodynamics and kinetics

- biochemistry

- scientific writing

\section{IT skills:}

- MS Office

- scientific software: ChemDraw, Origin, SciFinder, MestReNova

- various instrument software

\section{Languages:}

- English (fluent)

- French (native)

- German and Spanish (basics) 


\section{INTERESTS}

- Mountaineering (SAC member since 2009), hiking, team sports (football 12 years, rugby 3 years), rock-climbing

- Traveling (Europe, Africa, Asia, America)

- Drawing and painting (organization of art events, design of logos)

- Volunteer work (Lebanon, Romania, France)

\section{PUBLICATIONS AND PRESENTATIONS}

\section{Peer reviewed articles:}

- E. Cabane, V. Malinova, S. Menon, C. Palivan, and W. Meier, "Photoresponsive Polymersomes as Smart, Triggerable Nanocarriers", Soft Matter, 2011, DOI: 10.1039/C1SM05880K.

- E. Cabane, V. Malinova and W. Meier, "Synthesis of Photocleavable Amphiphilic Block Copolymer: Toward the Design of Photosensitive Nanocarriers", Macromolecular Chemistry and Physics, 2010, 211(17), 1847-1856.

- E. Cabane, Z. Boukhal, J. Claverie and Y. Durant, "Nanoparticle mediated oral delivery of insulin", Polymer Preprints (American Chemical Society, Division of Polymer Chemistry), 2007, 48(2), 946-947.

- Z. Boukhal, E. Cabane and Y. Durant, "Block Copolymer Characterization", PMSE Preprints, 2007, 97, 524-525.

- M. Zhang, E. Cabane, and J. Claverie , "Transparent Antifouling Coatings via Nanoencapsulation of a Biocide", Journal of Applied Polymer Science, 2007, 105(6), 3826-3833.

- E. Cabane, M. Zhang, and J. Claverie, "Preparation of Transparent Antifouling Coatings by Nanoencapsulation of an Antifouling Agent in a Transparent Matrix", Polymer Preprints (American Chemical Society, Division of Polymer Chemistry), 2005, 46(2), 1284-1285.

\section{Oral Presentations:}

- "Photocleavable Amphiphilic Block Copolymer: Toward the Design of Light Responsive Nanocarriers", EUPOC 2010, Gargnano, May 30-June 4, 2010.

- "Nanoparticle Mediated Delivery of Insulin", $3^{\text {rd }}$ Annual Materials Science Workshop, University of New Hampshire, November $1^{\text {st }}, 2006$.

- "Biocompatible Triblock Copolymer Self-Assembled in Vesicles: Application to Drug Delivery”, Annual Project Meeting, Bentley Pharmaceuticals, June 30, 2006.

- "Preparation of Transparent Antifouling Coatings by Nanoencapsulation of an Antifouling Agent in a Transparent Matrix", 230 ${ }^{\text {th }}$ National ACS Meeting, Washington DC, August 28-September 1, 2005. 


\section{REFERENCES}

- Prof. Dr. Wolfgang Meier

$\triangle \quad$ wolfgang.meier@unibas.ch

笋 $+41(0) 612673802$

Department of Chemistry

University of Basel

Klingelbergstrasse 80

CH-4056 Basel, Switzerland

- Dr. Nico Bruns

$\triangle$ nico.bruns@unibas.ch

缁 +41(0)6126738 32

Department of Chemistry

University of Basel

Klingelbergstrasse 80

CH-4056 Basel, Switzerland 